\title{
Growth and Properties of Magnetic Thin Films using Magnetron Sputtering Deposition
}

\author{
Manli Ding \\ Huaibei, Anhui, China \\ Master of Arts, University of Virginia, 2009 \\ Bachelor of Science, University of Science and Technology of China, 2007
}

A Dissertation presented to the Graduate Faculty of the University of Virginia in Candidacy for the Degree of

Doctor of Philosophy

Department of Physics

University of Virginia

April, 2013

Approved by the Examining Committee:

Prof. Joseph Poon, Thesis Adviser

Prof. Jiwei Lu, Member

Prof. Eugene Kolomeisky, Member

Prof. Utpal Chatterjee, Member 


\section{CONTENTS}

Growth and Properties of Magnetic Thin Films using Magnetron Sputtering

Deposition......i

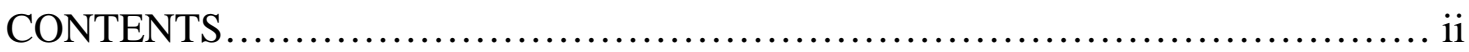

LIST OF TABLES ........................................................

LIST OF FIGURES.....................................................vi

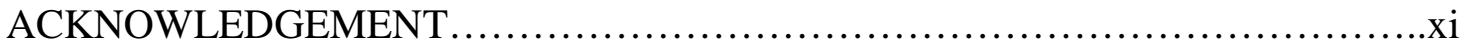

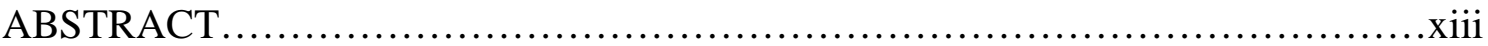

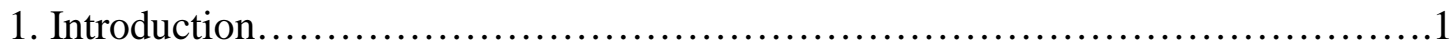

1.1 Motivation................................................................

1.2 Dissertation Outline......................................................2

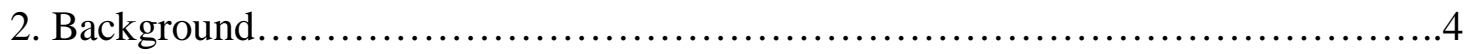

2.1 Spin Polarized Current...................................................4

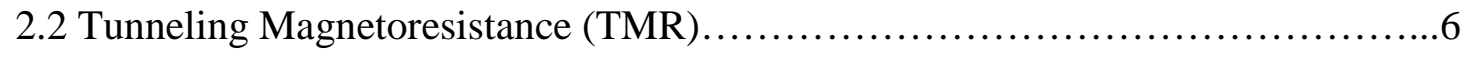

2.2.1 Magnetic Tunnel Junctions.........................................6

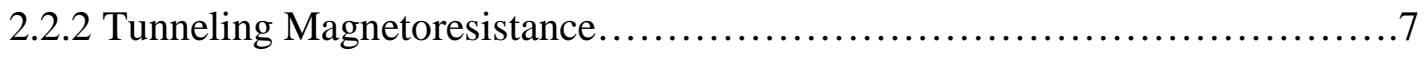

2.3 Current-induced Magnetization switching................................9

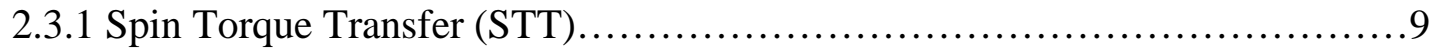

2.3.2 Precession of spin around local magnetization..........................11

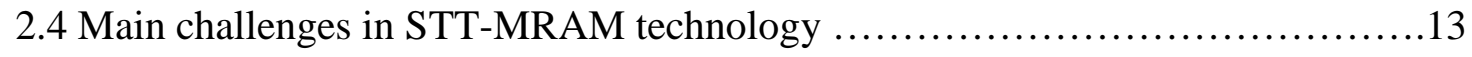

3. Experiment techniques ................................................

3.1 Introduction.............................................................. 
3.2 Magnetron Sputtering System................................................15

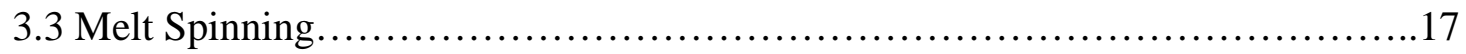

3.4 Sample Preparations..................................................... 18

3.4.1 Substrate cleaning......................................................

3.4.2 Post-deposition annealing............................................... 18

3.5 Film Characterization techniques..........................................19

3.5.1 Vibrating Sample Magnetometer (VSM).................................19

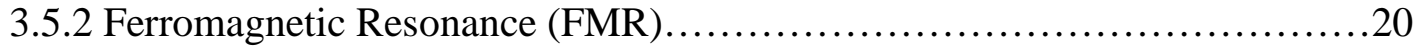

3.5.3 X-Ray Diffraction (XRD) / X-Ray Reflectivity (XRR).....................22

4. Cr-doped CoFeB films.........................................................

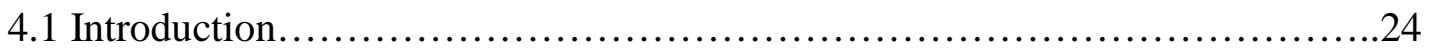

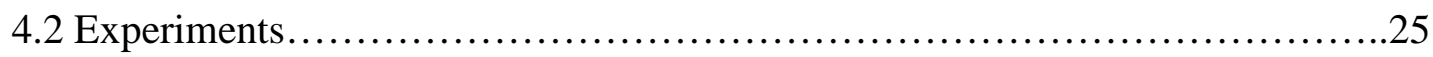

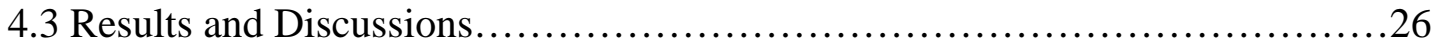

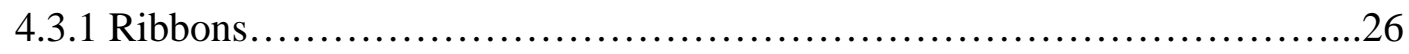

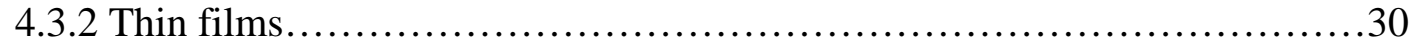

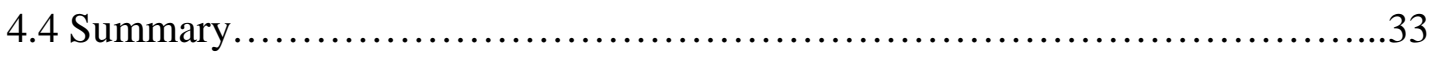

5. Interfacial perpendicular magnetic anisotropy in $\mathrm{CoFeGe/MgO} \mathrm{structure...........35}$

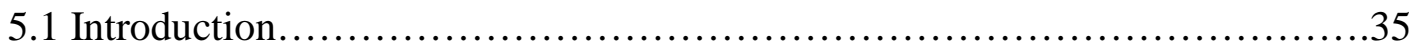

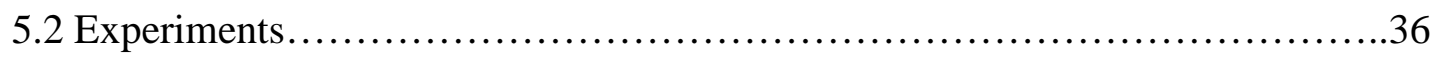

5.3 Results and Discussion..........................................................

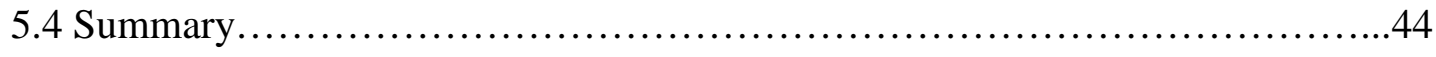

6. Crystalline alloys with perpendicular magnetic anisotropy.......................45 


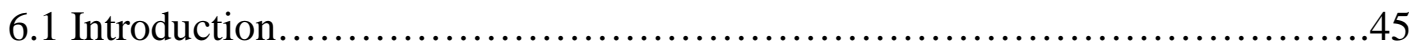

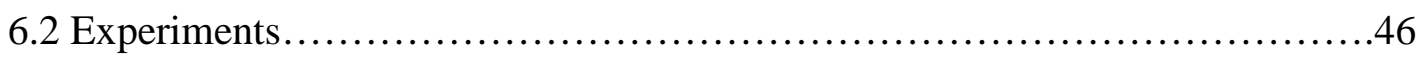

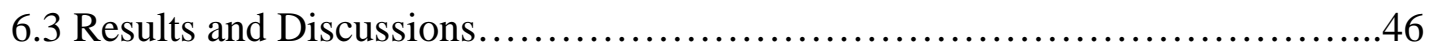

7. Tunable magnetic anisotropy in amorphous ferromagnetic GdFeCo films........50

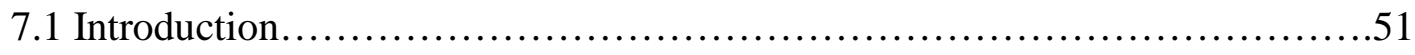

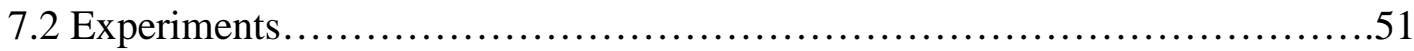

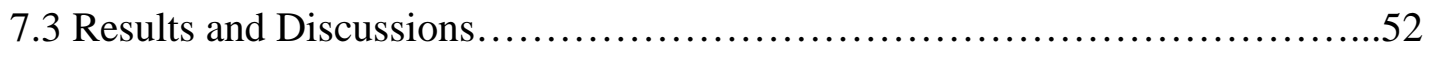

7.3.1 Low Gd-content films..............................................52

7.3.2 High Gd-content films............................................55

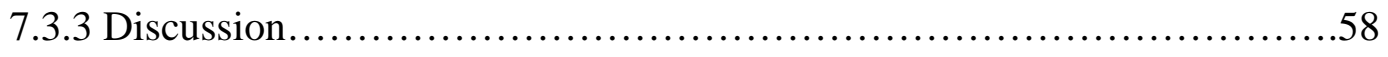

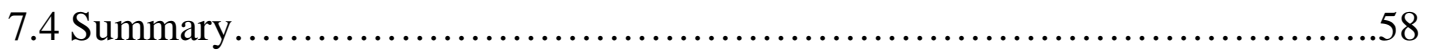

8. Conclusions and Future work.............................................60

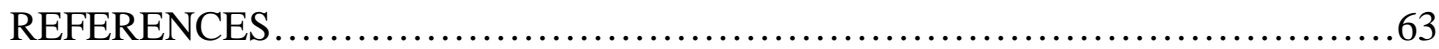




\section{LIST OF TABLES}

Table 4.1 Summary of the onset temperatures $\left(T_{x}\right)$ of crystallization phases determined by DSC measurements and saturation magnetization $\left(M_{s}\right)$ for as-spun $\mathrm{Co}_{40} \mathrm{Fe}_{\mathrm{x}} \mathrm{Cr}_{\mathrm{y}} \mathrm{B}_{\mathrm{z}}$

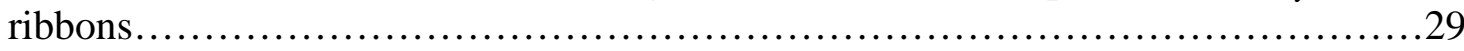




\section{LIST OF FIGURES}

Figure 2.1 Classical illustration of electron spin as a small magnet with north pole

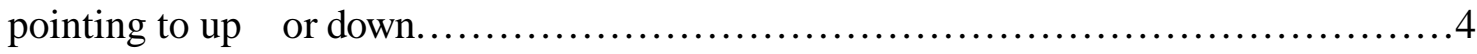

Fig 2.2 Diagram of the spin-resolved electronic structure for $4 \mathrm{~s}$ and $3 \mathrm{~d}$ bands in Co. Red (or blue) is for spin-down (or spin-up). Numbers denote how many electrons per Co atom are in the corresponding band...................................

Fig. 2.3 Illustration of the Jullière's model in a magnetic tunnel junction for parallel configuration (left) and antiparallel configuration (right). The current is determined by the product of the density of filled states in one electrode and the density of empty states in the other electrode. Parallel configuration usually has lower resistance than that of antiparallel configuration, thus resulting in a positive TMR

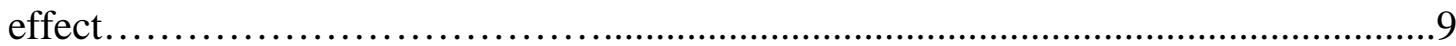

Fig. 2.4 The thick electrode is pinned layer (PL), and the thin one is free layer (FL). When electrons flow from PL to FL, due to spin transfer torque effect, it favors parallel state (left); on the other hand, when electrons flow from FL to PL, it favors antiparallel

state

Fig. 2.5 (a) Geometric illustration of Landau-Lifshitz-Gilbert (LLG) equation with a spin transfer torque term; (b) Dynamics of the magnetic moments in the free layer (FL) when the damping term is larger, comparable, or smaller than the spin-torque term with various magnitudes of spin polarized current.................................12

Figure 3.1 Magnetron Sputtering System.........................................16

Figure 3.2 Diagram of the melt spinning apparatus..............................17 
Figure 3.3 A field-swept FMR experimental system diagram......................21

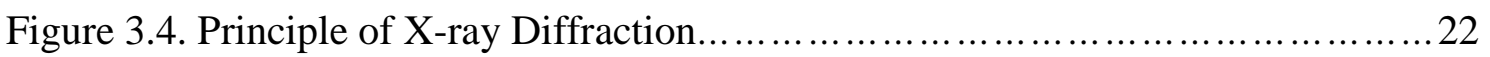

Figure 4.1 (a) XRD pattern and (b) Bright-field TEM image of as-spun $\mathrm{Co}_{40} \mathrm{Fe}_{32} \mathrm{Cr}_{10} \mathrm{~B}_{18}$ ribbon; The inset of Fig. (b) shows the selected area diffraction pattern...............27

Figure 4.2 (a) DSC curves of as-spun $\mathrm{Co}_{40} \mathrm{Fe}_{\mathrm{x}} \mathrm{Cr}_{\mathrm{y}} \mathrm{B}_{\mathrm{z}}$ ribbons with $\mathrm{y}=0,\{\mathrm{x}, \mathrm{z}\}=\{40,20\}$ and $y=10,\{x, z\}=\{30,20\},\{32,18\}$. (b) XRD patterns of $\mathrm{Co}_{40} \mathrm{Fe}_{32} \mathrm{Cr}_{10} \mathrm{~B}_{18}$ ribbons after annealing up to the onset of second crystallization stage.....................28 Figure 4.3 XRD $\theta-2 \theta$ scans of $\mathrm{Co}_{40} \mathrm{Fe}_{20} \mathrm{Cr}_{30} \mathrm{~B}_{10}(30 \mathrm{~nm})$ films annealed at temperatures

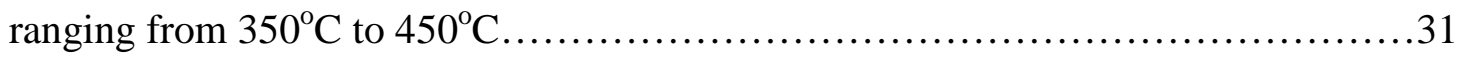

Figure 4.4 The impact from Cr content on the (a) saturation magnetizations and (b) coercive fields for both as-deposited and annealed films with annealing treatments at various temperatures....................................................32

Figure 5.1 (a) XRD $\theta-2 \theta$ scan of $\mathrm{Co}_{20} \mathrm{Fe}_{50} \mathrm{Ge}_{30}(20 \mathrm{~nm})$ film on $\mathrm{MgO}(001)$. (b) $\Phi$ scan on the $\mathrm{Co}_{20} \mathrm{Fe}_{50} \mathrm{Ge}_{30}(20 \mathrm{~nm})$ sample. The red line represents data taken at $2 \theta$ of $44.66^{\circ}$ (CoFeGe (022) peak) and the black one represents data taken at 62.45 ${ }^{\circ}$ (MgO (022)

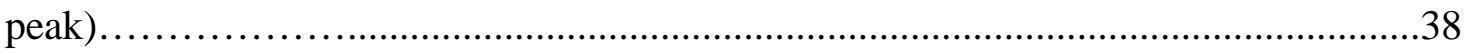

Figure 5.2 In-plane (black) and out-of-plane (red) magnetic hysteresis loops for (a) 1.2 $\mathrm{nm}$ and (b) $2 \mathrm{~nm}$ annealed $\mathrm{Co}_{20} \mathrm{Fe}_{50} \mathrm{Ge}_{30}$ films, respectively...

Figure 5.3 Saturation magnetization $M_{s}(\mathrm{emu} / \mathrm{cc})$ (red) and magnetization per unit area $m_{s}$ $\left(\mathrm{emu} / \mathrm{cm}^{2}\right.$ ) (blue) vs the $\mathrm{CoFeGe}$ film thickness at $300 \mathrm{~K}$ .40

Figure 5.4 The dependence of total perpendicular anisotropy $K$ on film thickness, plotted as the product of $\mathrm{K}$ and thickness vs. thickness 
Figure 5.5 In-plane (black) and out-of-plane (red) hysteresis loops for the annealed sample of $\mathrm{MgO}(001) / \mathrm{CoFeGe}(1.0 \mathrm{~nm}) / \mathrm{MgO}(2.0 \mathrm{~nm}) / \mathrm{Ta}(5 \mathrm{~nm})$....

Figure. 5.6 Frequency dependence of the linewidth for a $2.5 \mathrm{~nm}$ thick $\mathrm{Co}_{20} \mathrm{Fe}_{50} \mathrm{Ge}_{30}$ alloy film (annealed for $2 \mathrm{~h}$ at $250{ }^{\circ} \mathrm{C}$ ) with the field applied in the film plane and along the film normal (inset).

Figure 6.1 Lattice units of $\mathrm{D}_{22}-\mathrm{Mn}_{2.5} \mathrm{Ga}$, arrows stand for magnetic moment directions. .45

Figure 6.2 XRD $\theta-2 \theta$ pattern of $50-\mathrm{nm} \mathrm{Mn}_{2.5} \mathrm{Ga}$ film prepared at the substrate temperature of $400^{\circ} \mathrm{C}$ .46

Figure 6.3 $\Phi$-scan of the $\mathrm{Mn}_{2.5} \mathrm{Ga} / \mathrm{Cr} / \mathrm{MgO}$ structure in which $\mathrm{Mn}_{2.5} \mathrm{Ga}$ layer was prepared at $400^{\circ} \mathrm{C}$ .47

Figure 6.4 In-plane and out-of-plane M-H curves of the epitaxial Mn2.5Ga film prepared at $400^{\circ} \mathrm{C}$ .48

Figure 6.5 Saturation magnetizations $\left(\mathrm{M}_{\mathrm{s}}\right)$ of $\mathrm{MnGa}$ films deposited at different temperatures 49

Figure 7.1 (a) Cross-sectional TEM image of as-deposited $\mathrm{Gd}_{22} \mathrm{Fe}_{71} \mathrm{Co}_{7}$ film on $\mathrm{SiO}_{2} / \mathrm{Si}$ substrate. $6 \mathrm{~nm} \mathrm{MgO}$ layer was used to cap the film; (b) high-resolution TEM image of the $\mathrm{Gd}_{22} \mathrm{Fe}_{71} \mathrm{Co}_{7}$ film. The inset is a FFT pattern of the image.... .52

Figure 7.2 Temperature dependence of the saturation magnetization of as-deposited GdFeCo films with various Gd concentrations ( $\mathrm{x}=22,27,30$ and 35). The inset in (b) shows the dependence of the magnetization compensation temperature $T_{\text {comp }}$ on the Gd concentration. 
Figure 7.3 (a) Normalized out-of-plane hysteresis loops of as-deposited $\mathrm{Gd}_{27} \mathrm{Fe}_{66} \mathrm{Co}_{7}$ film measured at 250, 300, and 350 K. (b) In-plane (black square) and out-of plane (red circle) hysteresis loops of $50 \mathrm{~nm}$ as-deposited $\mathrm{Gd}_{22} \mathrm{Fe}_{71} \mathrm{Co}_{7}$ film.........................54

Figure 7.4 Temperature dependence of the saturation magnetization of GdFeCo films

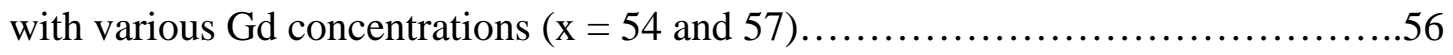
Figure 7.5 (a) Gd-content dependence of $\mathrm{M}_{\mathrm{s}}$ (black triangle) and $\mathrm{H}_{\mathrm{c}}$ (blue circle) for perpendicularly magnetized GdFeCo films between 52 and 59 at. \% Gd. (b) In-plane and out-of-plane hysteresis loops of $\mathrm{Gd}_{57} \mathrm{Fe}_{36} \mathrm{Co}_{7}$ film with thickness at $50 \mathrm{~nm} \ldots \ldots \ldots \ldots . . .57$ 


\section{LIST OF ABBREVIATIONS}

1. RE: Rare earth

2. FL: Free layer

3. FM: Ferromagnetic / Ferromagnet

4. TM: Transition metal

5. DOS: Density of States

6. DRAM: Dynamic Random Access Memory

7. GMR: Giant Magneto-Resistance

8. MR: Magneto-Resistance

9. MRAM: Magnetic Random Access Memory

10. MTJs: Magnetic Tunnel Junctions

11. PMA: Perpendicular Magnetic Anisotropy

12. RTA: Rapid Thermal Annealing

13. STT: Spin Torque Transfer

14. STT-RAM: Spin Torque Transfer Random Access Memory

15. TMR: Tunneling Magneto-Resistance 


\section{ACKNOWLEDGEMENT}

The completion of my dissertation and the underlying graduate research work would not be possible without the help and support from many people. First and foremost I would like to thank my advisor Prof. S. Joseph Poon, for his unconditional support and outstanding mentorship throughout my graduate research career. Next, I would like to thank Prof. Jiwei Lu for his professional guidance at the initial stage of my research, and the stimulating discussion and advices throughout my whole graduate research period. I would like to thank all of the former and present members in my research group, including Sreeparna Bhattacharya, Jack Simonson, Wu Di, Xiaopu Li, Andrew Cheung, Alex Petersen and Long Chen for making our lab such an enjoyable place to work every day. They are not only good team workers but also good friends in life. A special thank gives to all the co-researchers, including Wenjing Yin, Wei Chen, Peiyong Hang, Mandy Gu, Yishen Cui and Nattawut Anuniwat for the help over the past five years. I also would like to give acknowledgement to all the members of the faculty and staff in Physics Department, particularly Tammie Shifflett, Dawn Shifflett, Vicky Ingram for all the supporting work that they have done these years for my research and life in UVA. I would like to express my deep appreciation to our collaborator group of Prof. Tim Mewes in University of Alabama for his help on Ferromagnetic Resonance (FMR) measurements as well as helpful discussions in the past two years, and similar appreciation goes to Dr. Paul Adl at Micron for his help on compositional analysis as well.

Finally and most importantly, the unconditional love from my family has been the 
strongest support for my pursuit of graduate study and the PhD degree abroad. In particular, I would like to thank my dearest husband Wei for his selfless love and support. The past five years in Charlottesville with his accompany would be one chapter of my life that I cherish the most. To Wei and my dearest parents, I dedicate this dissertation work. 


\begin{abstract}
Spin-Torque-Transfer Magnetic Random Access Memory (STT-MRAM) has the potential to become a universal memory due to its superior properties compared with conventional memory technologies. However, the main challenge for implementing STT writing mode in high-density and high-speed memory is the substantial reduction of the intrinsic current density $J_{c 0}$ required to switch the magnetization of the free layer (FL) while maintaining high thermal stability required for long-term data retention.

The intrinsic switching current reduction can be pursued by using materials with low saturation magnetization $\left(M_{s}\right)$, low damping constant $(\alpha)$, high spin polarization $(\eta)$ and high magnetic anisotropy energy density $\left(K_{u}\right)$ at low dimension. Previous experiments showed that $\mathrm{CoFeB}$ incorporated in MgO-based MTJ is one of the most efficient materials for this purpose. In this work chromium was substituted into CoFeB alloys to further reduce their magnetization attributed to the antiferromagnetism of Cr. Films in composition of $\mathrm{Cr}$ at $30 \%$ showed a reduced magnetization of $300 \mathrm{emu} / \mathrm{cm}^{3}$ and an enhanced coercive field of 500 Oe, making this material suitable for device application.
\end{abstract}

Moreover, the switching current density $J_{c 0}$ is predicted to be reduced significantly by using materials with perpendicular magnetic anisotropy (PMA), compared with the normal in-plane ones. Perpendicular Magnetic anisotropy was found in crystalline $\mathrm{DO}_{22} \mathrm{Mn}_{2.5} \mathrm{Ga}$ films with optimal magnetic anisotropy energy of $0.5 \times 10^{7} \mathrm{erg} / \mathrm{cm}^{3}$, which leads to high thermal stability. A low saturation moment $\left(M_{s}\right.$ $=260 \mathrm{emu} / \mathrm{cc}$ ) was investigated, attributed to the strong antiferromagntic coupling 
between $\mathrm{Mn}$ atoms at different sites. With a reported high spin polarization of $88 \%, \mathrm{D0}_{22}-\mathrm{Mn}_{2.5} \mathrm{Ga}$ film has the potential to be used in spintronic devices.

Amorphous ferrimagnetic $\mathrm{Gd}_{\mathrm{x}} \mathrm{Fe}_{93-\mathrm{x}} \mathrm{Co}_{7}$ films were also found to exhibit low magnetization and perpendicular magnetic anisotropy, which is attributed to the competition between antiferromagnetic coupling of rare-earth (RE) with transitionmetal (TM) ions and ferromagnetic interaction between the TM ions. Furthermore, low magnetization was achieved at room temperature in previously unreported highGd region of $\mathrm{x}=52-59$, accompanied by a large PMA with coercivity reaching 6.6 kOe. Our findings have broadened the composition range of transition metal-rare earth alloys for designing PMA films, making it attractive for tunable magnetic anisotropy in nanoscale devices.

In addition to $M_{s}$ and $\eta$, one can also reduce FL intrinsic damping constant for lowering $J_{c 0}$, but practically PMA films often have higher damping constant $\alpha$ due to strong spin-orbit coupling. With reported low damping constant of 0.0025, CoFeGe film is promising spintronic material. Perpendicular magnetic anisotropy was achieved in the $\mathrm{MgO} / \mathrm{CoFeGe} / \mathrm{MgO}$ structure with an optimized magnetic anisotropy energy density of $2 \times 10^{6} \mathrm{erg} / \mathrm{cm}^{3}$, which is contributed by the interfacial anisotropy between CoFeGe and MgO. The $\mathrm{B}_{2}$-ordered CoFeGe is not half metallic but has a perfectly spin-polarized $\Delta_{1}$ band along the [001] direction, thus the perfect spin transfer efficiency with MgO barrier is expected in CoFeGe-base MTJs. 


\subsection{Motivation}

\section{INTRODUCTION}

The ability to manipulate the intrinsic spin of conduction electron and its associated magnetic moment, in addition to the fundamental charge, inspired an exciting new technology of spintronics [1]. Spintronics, a joint word of spin and electronics, emerged with the discovery of the giant magnetoresistance (GMR) effect [2]. GMR effect is the change of resistance that depends on the relative orientation of the magnetization of two ferromagnetic (FM) layers in response to an external magnetic field. Nevertheless, a higher magnetoresistance response is still demanded to further expand the application of spintronics devices. This eventually led to another big step in spintronics, the discovery of magnetic tunnel junction (MTJ). MTJ exhibits the behavior of tunneling magnetoresistance (TMR) with a much higher MR ratio than the GMR structure.

The development of spintronics coincides with the progress of semiconductor memories. In this information era, the demand for information storage is increasing but the devices that hold the memories become even more compact. However, cost effectiveness permits only little change on the existing complementary metal oxide silicon (CMOS) processing [3]. Moreover, the most important factor that triggers the search for a new memory technology is the inherent scalability issue in the current memory technologies. Therefore, the reason for implementing MTJ as a magnetic element in a magnetic memory device, or better known as magnetic random access memory (MRAM), becomes obvious. MRAM is said to be capable of the speed of static random access memory (SRAM), the density of dynamic random access memory (DRAM) [4], the non-volatility benefits of flash memory, and the unlimited endurance 
[5]. In other words, MRAM has the potential to be a universal memory.

However, after years of effort, the disadvantages of MRAM, such as large cell size and high write current, greatly forbids it to be widely commercialized [6]. More recently, a new phenomenon called spin transfer torque switching (STT) has been theoretically predicted and experimentally confirmed, which can be manipulated for producing a current-induced magnetization switching memory device. STT writing technology, by directly passing a current through MTJ, overcomes those hurdles with much lower switching current (in the order of $\mu \mathrm{A}$ ), simpler cell architecture and reduced manufacturing cost, and more importantly, excellent scalability to future technology nodes.

The main challenge for implementing STT writing mode in high-density and highspeed memory is the substantial reduction of the intrinsic current density required to switch the magnetization of the free layer while maintaining high thermal stability required for long-term data retention. To achieve these two goals, materials possessing low magnetization, high spin polarization, low damping constant and high magnetic anisotropy energy density are highly desired, which were explored in this dissertation with the results and discussions presented hereafter. Recent experiments showed that CoFeB incorporated in MgO MTJs is one of the most efficient materials for the purpose above. In our work chromium was substituted into $\mathrm{CoFeB}$ alloys to further reduce their magnetization. Moreover, the switching current density $J_{c 0}$ is predicted to be reduced significantly by using materials with perpendicular magnetic anisotropy (PMA), compared with the normal in-plane ones. Therefore, we have investigated three classes of material schemes with perpendicular magnetic anisotropy: (i) crystalline alloys 
(MnGa), (ii) materials with interfacial perpendicular magnetic anisotropy

(CoFeGe/MgO), and (iii) amorphous rare earth-transition metal (RE-TM) alloys

(GdFeCo). These material systems have been extensively characterized both magnetically and structurally. Their perpendicular magnetic anisotropy have been estimated, as well as the damping parameter, with the goal to find a material scheme with low damping, high magnetic anisotropy which will yield high performance MTJs for the application in STT-MRAM. 


\section{BACKGROUND}

\subsection{Spin polarized current}

As well as mass and charge, an electron has another intrinsic property, i.e. spin. In quantum mechanics, spin is a fundamental property of atomic nuclei and elementary particles, and is an important intrinsic degree of freedom. It had been shown by Dirac that electron spin arises naturally within relativistic quantum mechanics. The component of electron spin measured along any direction can only take on the values,

$$
\hbar s_{Z}, s_{Z}= \pm 1 / 2
$$

Namely, in a classical picture, electron spin can only point to up or down along a magnetic field which can be vividly illustrated by a small magnet with its north pole pointing to up or down as shown in Fig. 2.1.
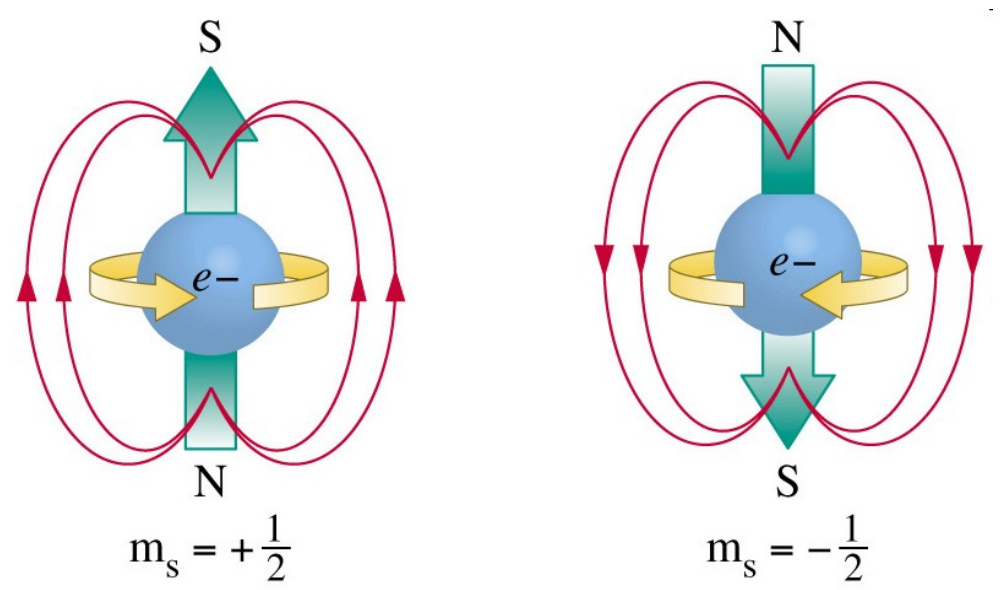

Fig. 2.1 Classical illustration of electron spin as a small magnet with north pole pointing to up or down.(adapted from Ref[6])

In normal materials, such as $\mathrm{Cu}$, Ag, etc., spin-up and spin-down electrons are equally populated and randomly distributed in an equilibrium state. However, in some solids due to the quantum mechanical exchange interaction, electron spins are aligned spontaneously, resulting in unequal numbers of spin-up and spin-down 
electrons, therefore ferromagnetic materials are formed, such as Co, Fe and many of their alloys. In these ferromagnetic materials, spin-resolved electronic structures show the band difference between the spin-up and spin-down electrons, as illustrated in Fig. 2.2, and the asymmetry in density of states (DOS) at Fermi energy gives rise to most of the spin related transport phenomena. When an unpolarized flow of electrons pass through a normal metal, because of the indemnity between the bands for spin-up and spin-down electrons, they experience the same scattering rate regardless of the spin, and the emergent current still remains unpolarized. However, when they flow through ferromagnetic material, spin-up electrons may encounter less scattering, or may have smaller effective mass, compared to spin-down electrons, so the conductivity due to the spin-up channel would be higher. Then a spin polarized current would emerge from the ferromagnetic material [6].

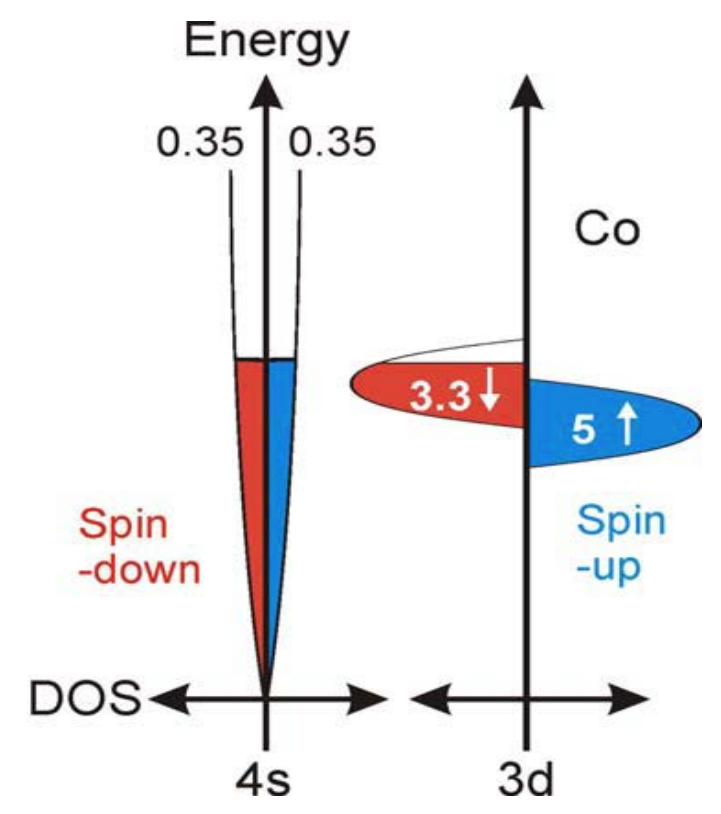

Fig 2.2 Diagram of the spin-resolved electronic structure for $4 \mathrm{~s}$ and $3 d$ bands in Co. Red (or blue) is for spin-down (or spin-up). Numbers denote how many electrons per Co atom are in the corresponding band. (Adapted from Spintronics lecture notes, Prof. Stu Wolf, UVA 2013) 
Such a spin polarized current is of significant importance because it can be used to manipulate the magnetization orientations in magnetic multilayer nanostructures via spin, instead of electron charge only in conventional electronics [7]. Since the discovery of the giant magnetoresistance effect in 1980's [8-10], spin-electronics (spintronics) using electron spin to control the current has stimulated great interest in both the academic and industrial fields. A few years later, an even more significant effect, called the tunneling magnetoresistance (TMR) effect, was observed at room temperature in magnetic tunneling junctions (MTJs) [11-14]. Because of their huge TMR (above 200\%), MTJs promise attractive applications in magnetic random access memory (MRAM) [7]. This new type of memory has all the key desirable attributes required by the ever growing demand for storing and processing the explosive amount of information, such as fast access times, non-volatility, lower power-consumption, and high density [7]. There are many ongoing efforts to realize MRAM in recent years.

\subsection{Tunneling Magnetoresistance (TMR)}

\subsubsection{Magnetic tunnel junction (MTJ)}

Magnetic tunnel junctions (MTJs) are sandwiched heterostructures, composed of two ferromagnetic electrodes and an ultrathin insulating barrier in between. One of the two ferromagnetic (FM) layers has its magnetization fixed (referred as the fixed or pinned layer; in real case, it is pinned by an antiferromagnetic layer via exchange bias), while the magnetization of the other FM layer is free to rotate (free layer). MTJ 
structures present many interesting phenomena that are important for both the exploration of underlying physics and the applications in technology [15]. When electrons flow through a MTJ, they become spin-polarized by the first magnetic electrode. Thereafter, the interplay between the spin-polarized current and the second magnetic layer exhibits two effects, i.e. tunneling magnetoresistance effect and spin transfer torque effect. In the next section of this chapter, brief reviews will be given on these two interesting effects.

\subsubsection{Tunneling Magnetoresistance (TMR)}

The resistance of a MTJ depends on the relative alignment of the magnetic moments in two ferromagnetic electrodes. Usually, when the moments are parallel the tunneling resistance is low; while anti-parallel, the resistance is high, thus giving rise to a tunneling magnetoresistance (TMR) effect,

$$
T M R=\frac{R_{A P}-R_{P}}{R_{P}} \times 100 \%
$$

where $R_{P}$ and $R_{A P}$ are the resistances for the parallel and anti-parallel configurations. By cleverly engineering the multilayered MTJ structure, it is possible to control how two magnetic electrodes respond to the external field and therefore to obtain distinct states of different resistance values [16].

The first successful observation of TMR in MTJs was made by Jullière about thirty years ago, where Co and Fe were used as electrodes and Ge as insulator and a TMR of $\sim 14 \%$ at $4.2 \mathrm{~K}$ was reported [17]. After that many other tunnel barriers were explored (e.g. $\mathrm{NiO}$ and $\mathrm{Gd}_{2} \mathrm{O}_{3}$ ) [18,19], but only a small effect was observed even at a low temperature. It was not until 1995 when the first observation of reproducible large 
TMR ( 18\%) at room temperature was achieved in MTJs with an amorphous $\mathrm{Al}_{2} \mathrm{O}_{3}$ barrier [20,21]. Ever since then, MTJs have aroused considerable interest for potential applications in high-performance MRAM due to their large and adjustable resistances, which allow high-speed read operation in memory devices [15].

Although intensive efforts have been made in MTJs with an $\mathrm{Al}_{2} \mathrm{O}_{3}$ tunnel barrier [22-24], the maximum TMR only reached about 70\% [25,26]. Meanwhile, extensive theoretical and experimental work has been carried out to increase the TMR by incorporating exotic oxide or half-metal layers into MTJs [27-30], and studying crystalline tunneling barriers [31,32]. Butler et al. carried out first-principles calculations of tunneling conductance and magnetoresistance in epitaxial $\mathrm{Fe} / \mathrm{MgO} / \mathrm{Fe}$ sandwiches [31]. They revealed that tunneling conductance depends strongly on the symmetry of the Bloch states in the electrodes and of the evanescent states in the barrier, thus Bloch states of different symmetry decay at different rates within the barrier. The $\Delta_{1}$ state, due to its "s-character", decays slowest among all the states and can make a significant contribution to the tunneling conductance. Because the state only occurs at the Fermi level for the majority band, it behaves much like a "half-metal". Giant TMR was expected in bcc $\mathrm{Fe} / \mathrm{MgO} / \mathrm{Fe}$, $\mathrm{CoFe} / \mathrm{MgO} / \mathrm{CoFe}$, and $\mathrm{Co} / \mathrm{MgO} / \mathrm{Co}$ tunneling junctions. Experimental results of TMR above $~ 200 \%$ were first reported by Parkin et al. and Yuasa et al. respectively [33,34]. Room temperature TMR results of about 600\% in MgO based tunnel junctions have also been obtained recently [35].

For high-quality $\mathrm{Al}_{2} \mathrm{O}_{3}$ tunnel junctions, the magnitude of TMR at low bias can be well understood within the framework of Jullière's model, $T M R=\frac{2 P_{1} P_{2}}{1-P_{1} P_{2}}$, where $P_{1}, P_{2}$ are the spin polarization of the two electrode materials, which were well derived from the 
ferromagnet/insulator/conducting tunnel junctions first introduced by Meservy and Tedrow [36]. In this model, it is assumed that during the tunneling process, spin is conserved, namely no spin-flipping occurs. The conductance in each channel is thus proportional to the tunneling probability, which is determined by Fermi's golden rule (Fig. 2.3). However, such a quantitative comparison is not always straightforward. For example, TMR is extremely sensitive to the interface between the electrode and barrier in realistic systems [37,38]. In MgO-based MTJs, due to coherent tunneling effect, the physics of the spin dependent tunneling effect is far beyond this simplified model. The observed giant TMR in MgO-based MTJs originates from the fact that the electrons in highly spin-polarized $\Delta 1$ band in (001) direction of bcc ferromagnetic electrodes can tunnel through the MgO (001) barrier more easily than the electrons in other bands ( $\Delta 2$ and $\Delta 5$ ). For the coherent tunneling through a crystalline system many theoretical studies emerged together with the experimental achievements in MgO based tunnel junctions [31-34]. 

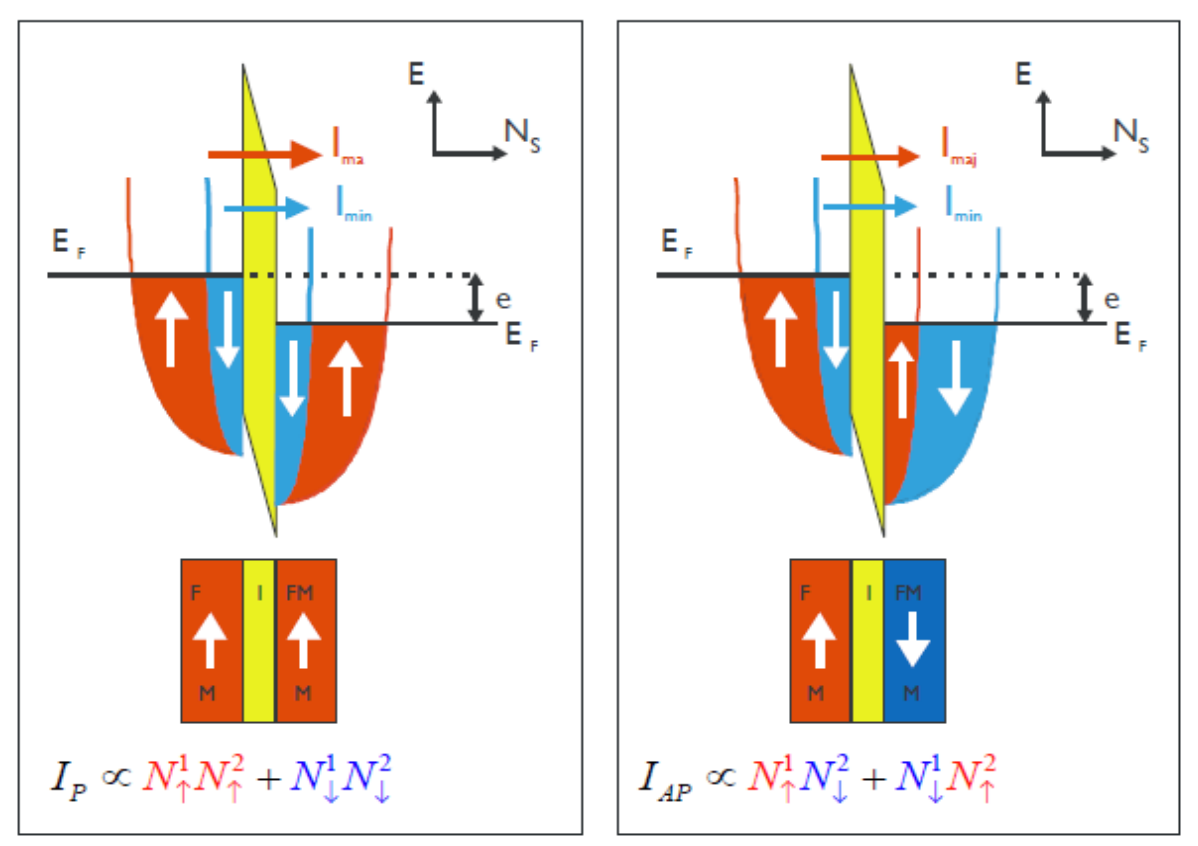

Fig. 2.3 Illustration of the Jullière's model in a magnetic tunnel junction for parallel configuration (left) and antiparallel configuration (right). The current is determined by the product of the density of filled states in one electrode and the density of empty states in the other electrode. Parallel configuration usually has lower resistance than that of antiparallel configuration, thus resulting in a positive TMR effect. (Adapted from Ref[36]))

\subsection{Current-induced Magnetization switching}

\subsubsection{Spin Transfer Torque (STT)}

Spin transfer torque (STT) effect was first theoretically studied by Slonczewski and Berger in 1996 [39,40]. Consider a basic MTJ structure with two ferromagnetic electrodes separated by a barrier layer [41]. The electrode of smaller thickness, whose magnetization can be switched, is called the free layer (FL); in contrast, the other electrode, which is usually thicker and exchange biased, is called pinned/fixed layer (PL). Moreover, there is an angle $\theta$ between the magnetization of two FM layers for current switching purpose. When the incoming spin orientation is collinear with the magnetization of the FM layer $(\theta=0$ or $\pi)$, there will be no torque exerted [42]. Since 
spin transfer is crucial in switching the magnetization of the free layer, therefore noncollinearity between fixed and free layer is necessary.

The spin transfer phenomena occur for electron current flowing through a MTJ structure. The current becomes spin polarized by transmission through or reflection from the first ferromagnetic layer (pinned layer) and mostly maintains this polarization as it passes through the barrier spacer, then enters and interacts with the second ferromagnetic layer (free layer). The spin of electrons will try to align with this local magnetization by precessing around it when the electrons move through the free layer. Since the precession is averaged over all electrons, transverse components of spin angular momentum become zero because the electrons are out of phase. Due to the conservation of spin angular momentum, those transverse components of electron spins will be absorbed and transferred to the conduction electrons of free layer. Therefore, the same interaction exerts a torque on the magnetization of free layer, making the magnetization tend to align towards the magnetization of the fixed layer. This torque effect is commonly known as spin transfer toque. Although the spin-down electrons, with respect to the free layer, will be reflected back to the fixed layer, the magnetization of the fixed layer will not change because this torque is not strong enough. The strength of the torque is normally expressed as the magnitude of incoming current density. If the current density is high enough, that is more than critical switching current (usually around $10^{7} \mathrm{~A} / \mathrm{cm}^{2}$ ), the torque applied by the spin of electrons can even reverse the magnetization of the free layer [42].

\subsubsection{Precession of spin around local magnetization}


Depending on the direction of electron flow and the magnetic configuration, this spin-polarized current favors either the parallel (P) state or antiparallel (AP) state (Fig. 2.4). Such a STT-induced magnetization reversal is a relatively new phenomenon, and it is observable only in magnetic structures smaller than 100-200 nm [43-45]. A macro-spin model treats a nanomagnet with the assumption that its internal magnetic degrees of freedom are frozen, so that the dynamics of macro-spin can be phenomenologically described by the following Landau-Lifshitz-Gilbert (LLG) equation with an extra STT term $[46,47]$ :

$$
\partial_{t} \vec{m}=-\gamma\left(\vec{m} \times \overrightarrow{H_{e f f}}\right)+\alpha\left(\vec{m} \times \partial_{t} \vec{m}\right)-\beta J(\vec{m} \times \vec{m} \times \vec{M})
$$

where $\gamma$ is the gyromagnetic ratio, $\alpha$ is the damping coefficient, $\beta$ is the coefficient for the STT which depends on both the spin polarization and the geometric configuration between the incoming spin and the local moments in the FL, and $J$ is the current density.
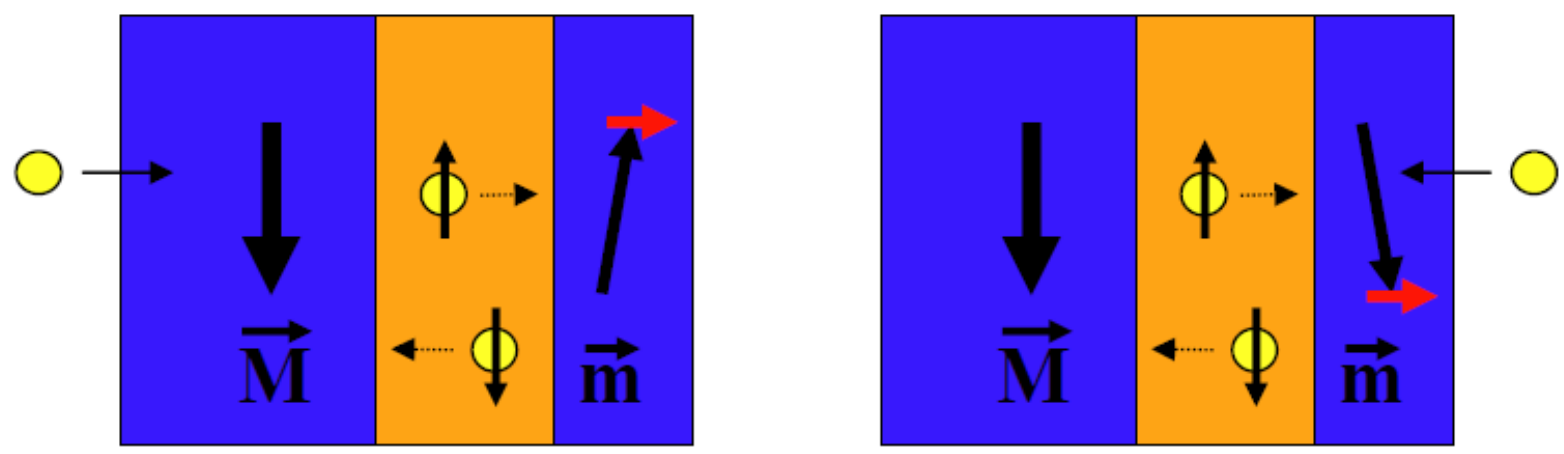

Fig. 2.4 The thick electrode is pinned layer (PL), and the thin one is free layer (FL). When electrons flow from PL to FL, due to spin transfer torque effect, it favors parallel state (left); on the other hand, when electrons flow from FL to PL, it favors antiparallel state. [Adapted from Reference [43]]

The competition between the damping term and the spin torque term is illustrated in Fig. 2.5. When $J$ is small and the spin torque term is smaller than the damping term, the dynamics damp out into an equilibrium state. When the spin torque is large enough 
that it overcomes the intrinsic damping, effective damping coefficient becomes negative, the deviation from the equilibrium state is amplified and the magnetic moments are switched, which can be detected by a resistance change in the MTJs. When $\mathrm{J}$ and $\mathrm{H}$ satisfy certain satisfy certain conditions, persistent precession of the magnetization can be obtained at a frequency of several GHz.

(a)

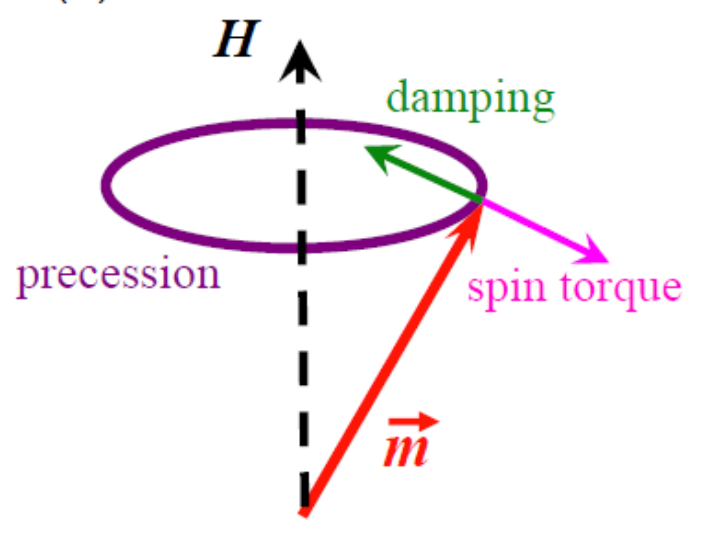

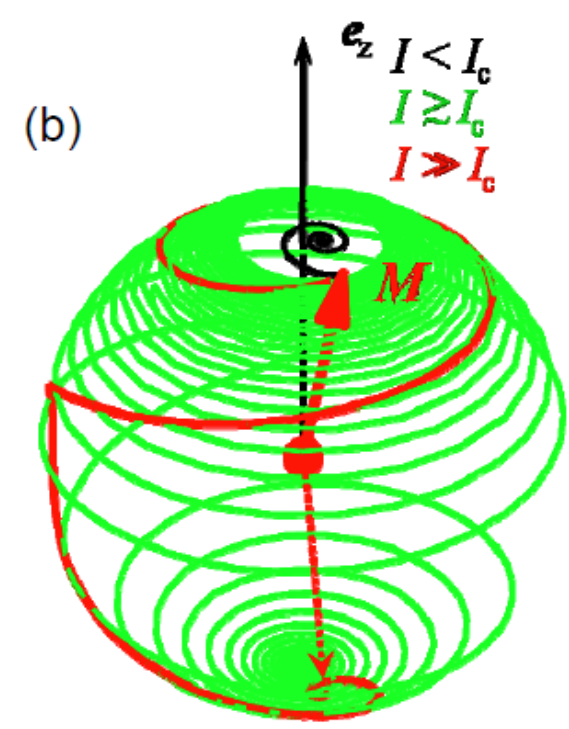

Fig. 2.5 (a) Geometric illustration of Landau-Lifshitz-Gilbert (LLG) equation with a spin transfer torque term; (b) Dynamics of the magnetic moments in the free layer (FL) when the damping term is larger, comparable, or smaller than the spin-torque term with various magnitudes of spin polarized current. [Adapted from the NRI e-workshop: "Spin Torque Transfer" 08/2009]

With these new phenomena in physics and potential applications in technology, the STT effect has stimulated great interest in both academics and industry since the beginning of its discovery. This is useful in the very small scale solid-state circuitry, and successful switching of the magnetization in MgO based MTJs has been observed. Besides, due to their much higher magnetoresistance effect, they are more desirable as memory storage cells which can tolerate greater error margins. The STT-MRAM has excellent write selectivity since the current passing through a storage cell can directly change the state of that cell, i.e. write " 0 " or " 1 " there. The minimum current density 
needed to write one bit scales down with the device size, which means that STT-MRAM has much higher scalability. Besides, STT-MRAM also promises lower power consumption and much simpler architecture.

\subsection{Main challenges in STT-MRAM technology}

The main challenges for implementing STT writing mode in high-density and high-speed memory is the substantial reduction of the intrinsic current density $J_{c 0}$ required to switch the magnetization of the free layer while maintaining high thermal stability required for long-term data retention. Minimal switching (write) current is required mainly for reducing the size of the selection transistor in series with MTJ in one transistor and one MTJ design (1T1J) to achieve the highest possible memory density, because the channel width of the transistor is proportional to the write current for a given transistor current drivability $(\mu \mathrm{A} / \mu \mathrm{m})$. Second, smaller voltage across MTJ decreases the probability of tunneling barrier degradation and breakdown, ensuring write endurance of the device.

The critical current density required to cause magnetization reversal in a MTJ at zero temperature $[48,49,50]$, using macrospin approximation is given as:

$$
J_{c 0}=\left(\frac{2 e}{\hbar}\right)\left(\frac{\alpha}{\eta}\right)\left(M_{s} t_{F}\right)\left(H_{e x t}+H_{k}+2 \pi M_{s}\right)
$$

where $H_{\text {ext }}$ is the field applied along the easy axis, $M_{s}$ and $t_{F}$ are the magnetization and thickness of free layer respectively, $\alpha$ is the damping constant, and $H_{k}$ is the effective anisotropy field including magnetocrystalline anisotropy and shape anisotropy. The spin transfer efficiency $\eta$, is a function of the current polarity, polarization, and the relative angle between the free and pinned layer. Eq. (1) gives a current density threshold. 
The thermal stability factor of the free layer is defined as:

$$
\Delta=\frac{K_{u} V}{k_{B} T}=H_{k} M_{s} V / 2 k_{B} T
$$

Where $\mathrm{K}_{\mathrm{u}} \mathrm{V}$ is the anisotropy energy.

The major objectives in this dissertation were to investigate and optimize various magnetic materials to be used as free layer in MgO-based MTJs for ultimate applications in STT-MRAM devices. Refereeing to Eq. (1) and (2), the reduction of the intrinsic switching $\mathrm{J}_{\mathrm{c} 0}$ while maintaining a high thermal stability can be pursed through materials engineering from the following two aspects:

(1)using materials with low magnetization $\mathrm{M}_{\mathrm{s}}$, low damping constant, a high spin transfer efficiency.

Recent experiments showed that CoFeB incorporated in MgO MTJs is one of the most efficient materials for this purpose. In this work chromium was substituted into CoFeB alloys to further reduce their magnetization.

(2)The most efficient means of reducing $\mathrm{J}_{\mathrm{c} 0}$ would be to use a free layer with perpendicular anisotropy with $H_{k \perp}>4 \pi \mathrm{M}_{\mathrm{s}}$. In this case, the magnetization is out of the film plane, which would eliminate the $2 \pi M_{s}$ term in the Eq. (1), where the $H_{k}$ term becomes the effective perpendicular anisotropy $H_{k}^{\perp}=H_{k \perp}-4 \pi \mathrm{M}_{\mathrm{s}}$ In addition, large magnetic anisotropy energy usually found in perpendicular FL provide significantly large thermal stability $\Delta$, thus sustainable at very small technology nodes. To this end, we have investigated three classes of material schemes with perpendicular magneti ansitropy: (i) crystalline alloys (MnGa), (ii) materials with interfacial perpendicular magnetic anisotropy (CoFeGe/MgO), and (iii) amorphous rare earthtransition metal (RE-TM) alloys (GdFeCo). These material systems have been 
extensively characterized both magnetically and structurally, and their damping parameters have been estimated, with the goal to find a low damping, high anisotropy material scheme which will yield high performance MTJs for the application in STTMRAM. 


\subsection{Introduction}

\section{Experimental Techniques}

A brief description of experimental techniques used during the development and characterization of film stacks is given in this chapter. All the films were deposited using magnetron sputtering system. The features of this tool, as described below, result in rapid and repeatable generation of complex depositions. The compositions of films were determined using inductively coupled plasma-mass spectrometry (ICP-MS) after chemically dissolving the films, and confirmed by X-ray fluorescence (XRF) using elemental standards. Structural characterization of films were performed by $\mathrm{x}$-ray diffraction $(\mathrm{XRD})$ with $\mathrm{Cu} K \alpha(\lambda=1.541 \AA)$ radiation (Smart-lab ${ }^{\circledR}$, Rigaku Inc.) and high resolution transmission electron microscopy (FEI, HRTEM). The thickness of the films was measured by X-Ray reflectivity. The film magnetic behaviors were characterized using vibrating sample magnetometer (VSM) and Magneto Optical Kerr effect (MOKE). The damping constants of films were characterized using an automated broadband ferromagnetic resonance (FMR) set-up custom built by Professor Tim Mewes from University of Alabama. Atomic Force Microscopy was used to characterize the film surface morphology.

\subsection{Magnetron Sputtering System}

Magnetron Sputtering is one of the most commonly used Physical Vapor deposition (PVD) approaches for thin film fabrication. It is similar to other simple DC sputtering systems, which initiate sputtering by applying an electric field between the target (cathode metal) and the substrate (anode). Specifically, stray electrons near the 
cathode are accelerated toward the anode under the electric field. Process gas atoms are ionized while colliding with the electrons. Electrons are accumulated during gas atom ionizations. Meanwhile, as the ions collide with the cathode, the secondary electrons are ejected contributing to the charge multiplication. A discharge finally occurs with a large avalanche current. Stable sputtering is initiated with enough ions starting bombarding the target.

Unlike DC sputtering systems, a magnetic field in close proximity of the target surface is employed to achieve a better secondary electron confinement in magnetron sputtering $[48,49]$. The magnetic field is roughly parallel with the target surface. The ionization of the gas atoms is greatly improved due to the hopping motion of the trapped electrons in the electric and magnetic fields during sputtering.

Compared with sputtering techniques such as simple DC sputtering, magnetron sputtering operates at a lower process pressure thus avoiding intense gas collisions and scatterings. Therefore a stable plasma and centain growth rate can be maintained with a relatively low target voltage $(\sim 500 \mathrm{~V})[50]$.

In our magnetron sputtering system as shown in Figure 3.1, the main process chamber is connected to a cryppump to maintain a base pressure below $1 \times 10^{-6}$ Torr. The main chamber accommodates four targets, three of which are operated with a RF bias wihle the fourth one can be operated with both DC and RF bias. Argon is used as the process gas and the process pressure is in the range of a few millitorr. The power of each target can be adjusted to vary the growth rate of the corresponding material or to modify the film composition in a co-sputtering process. The load lock is connected to a turbo 
pump. During sample loading the load lock is isolated from the main chamber by a gate valve.

Substrates are mounted on the sample holder using silver paint or heat resistant tape. The sample holder is later fitted into a fork assembly in the load lock. After sealing and pumping down the load lock chamber, the sample holder can be transferred into the main chamber and attached to the carousel, which allows the substrates to rotate facing down toward the target. The folk assembly also retrieves the sample holder back to the load lock after

deposition.

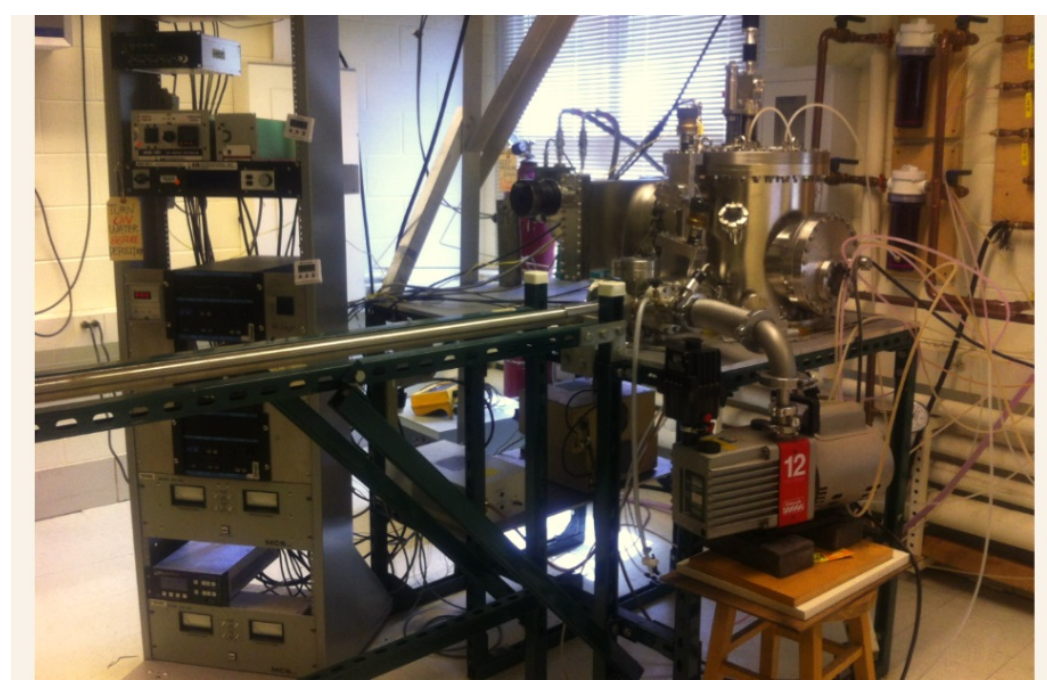

Figure 3.1 Magnetron Sputter System

The deposition rates for various materials at different sputtering conditions were determined by depositing fairly thick films on the substrates for thickness measurement. $\mathrm{X}$-ray reflectivity was used to measure the thickness of the films. Once sputtering rates 
were calculated from these thicknesses, the deposition times for various films deposited under the same power and pressure conditions could be calculated.

\subsection{Melt Spinning}

Melt-spinning is a technique for rapidly cooling of liquids in a single stage, as shown schematically in Fig. 3.2. A wheel is cooled internally by water, and rotated. A thin stream of liquid metal (Iron-based alloys in our study) is then dripped onto the wheel and cooled in a high purity argon atmosphere, causing rapid solidification. Ribbons are eventually spun from the wheel, generally having the thickness of $\sim 25 \mu \mathrm{m}$.

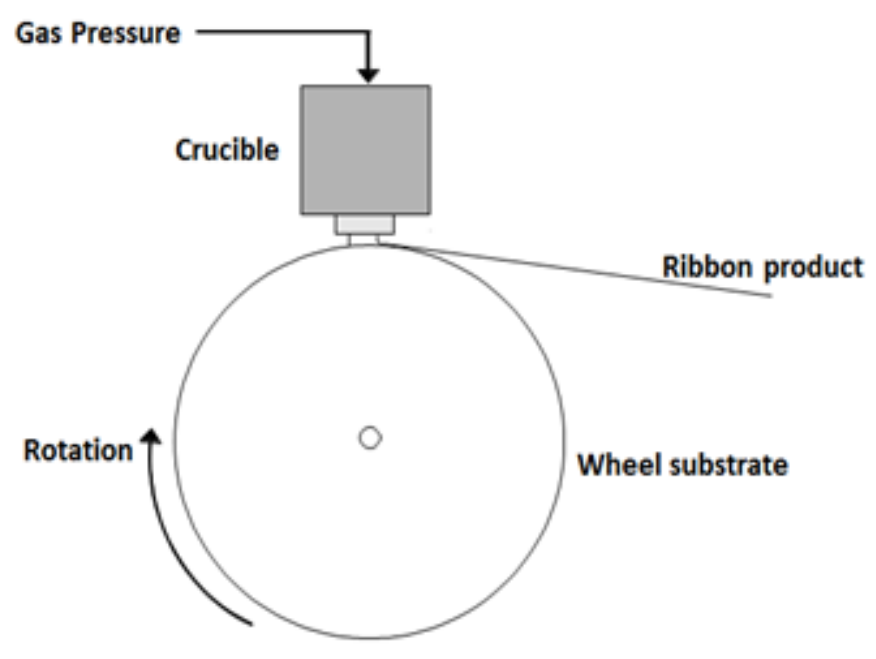

Figure 3.2 Diagram of the melt spinning apparatus.

Melt spinning system has been used in our study, primarily driven by the interest in producing amorphous as-spun ribbons via the high cooling rates obtained from this process.

\subsection{Sample Preparation}




\subsubsection{Substrate cleaning}

In any case, the substrate cleaning protocol as described in the following procedure was strictly followed for all the experiments. First step is the ultrasonic cleaning of substrates with an acetone solution for several minutes. One clean aluminum dish, which is used to hold the substrates and acetone solution during the first step of cleaning, is rinsed by acetone to make sure the tiny aluminum particles on the surface of the dish will be washed away and won't be mixed in the acetone during the ultrasonic process to avoid contaminating the substrate surfaces. After the ultrasonic cleaning with acetone, the substrates are picked out by a pair of clean nonmagnetic tweezers, rinsed in isopropanol to wash away the acetone residual and put into the second clean aluminum dish that is rinsed by isopropanol. The substrates are then immersed in isopropanol for another several-minutes ultrasonic cleaning process. After two ultrasonic cleaning steps, the substrates are then picked out and blown dry by pure nitrogen gas and put into another clean aluminum dish for the final baking process. The substrates are baked at $120^{\circ} \mathrm{C}$ on a hot plate to get rid of the surface moisture before putting into the vacuum chamber.

\subsubsection{Post-deposition annealing}

The post-deposition annealing treatment is essential for forming good crystalline phases of thin films. In order to anneal our samples with an external magnetic field capability, there is one vacuum furnace, which can provide annealing up to $500^{\circ} \mathrm{C}$ with very precise digital control of the temperature. The vacuum base pressure is in

the low $10^{-6}$ Torr range and a magnetic field up to 1400 Oe is provided by two surrounded permanent magnets. In terms of temperature control, this vacuum furnace 
has the ramp-up rate of $5^{\circ} \mathrm{C} / \mathrm{min}$, and slow natural cooling-down rate less than $2^{\circ} \mathrm{C} / \mathrm{min}$. Another method of heat treatment is the rapid thermal annealing (RTA), in which the temperature ramp-up and cooling rate is much faster compared with the traditional vacuum furnace. The RTA instrument (As-One 100) was equipped with an infrared lamp as the radiation heating source, and the temperature was monitored by a pyrometer underneath the sample stage. Both forming gas $\left(95 \% \mathrm{~N}_{2}, 5 \% \mathrm{H}_{2}\right)$ and vacuum (base pressure $\sim 10^{-6}$ Torr) modes can be used during the heating to protect films from oxidation. The RTA temperature ramp-up rate can easily reach $20^{\circ} \mathrm{C} / \mathrm{s}$, and the temperature is maintained very stably with the digital power control system during the annealing process. The step of cooling down from high temperature like $500^{\circ} \mathrm{C}$ also takes only few minutes with the RTA method.

\subsection{Films Characterization techniques}

\subsubsection{Magnetic Characterization}

The magnetic hysteresis loops were characterized using a Vibrating Sample Magnetometer (VSM). Additionally the magnetic anisotropy between two orthogonal directions can be estimated using the magnetization methods. In particular, the perpendicular magnetic anisotropy (PMA) in this work was estimated by characterizing the hysteresis loops in magnetic fields along directions in the film plane and perpendicular to the film plane. Based on the energy consumption to change the magnetization in an applied magnetic field, the total effective anisotropy energy $K_{\text {eff }}$ can be calculated by evaluating the area enclosed between the in-plane and perpendicular 
hysteresis loops[52,53]. In the cases where magnetic domains are switched coherently with few pinning sites, $K_{\text {eff }}$ can be equivalently obtained by [43, 86]:

$$
K_{e f f}=\frac{H_{K} M_{S}}{2}
$$

where the anisotropy field $H_{K}$ equals to the saturation field along with the hard axis. In other cases where a considerable hysteresis is shown, one has to first remove the hysteresis by averaging the hysteresis loop branches and then estimate the enclosed area. Besides, in the PMA case, the total effective magnetic anisotropy energy can be written as:

$$
K_{e f f}=K_{u}-2 \pi M_{S}^{2}
$$

$K_{u}$ is the uniaxial magnetic anisotropy that possibly originates from perpendicular crystalline anisotropy or interfacial anisotropy. $2 \pi M_{S}^{2}$ represents the in-plane shape anisotropy energy. Thus $K_{u}$ can be obtained by subtracting the contribution of shape anisotropy $2 \pi M_{S}^{2}$ from $K_{e f f}$.

The magnetic hysteresis loops are measured using the VSM option in Versalab (Quantum Design). This system is equipped with a superconducting magnet providing a maximum field up to 30000 Oe. The measurement temperature can be lowered to below $50 \mathrm{~K}$. To avoid any magnetic contamination, the samples were cleaned with acetome and isopropanol, dried using UHP $N_{2}$ and baked on a hot plate at 100 degree for 2 minutes. The samples holders were also cleaned with acetone and isopropanol for the same reason. The temperature is kept constant with a slow field sweep rate to maintain isothermal conditions during the measurements.

\subsubsection{Gilbert Damping constant characterization}


One of the most commonly used methods to characterize the Gilbert damping constant is through Ferromagnetic Resonance (FMR). Ferromagnetic resonance originates from the precession of the magnetization of a ferromagnet that can be welldescribed in terms of Landau-Lifshitz-Gilbert (LLG) equation [46,47]. The precession frequency held by the external field is given as $\omega=\gamma\left|H_{\text {ex }}\right|$. But it can be better described by Kittel formula in the case of thin films where the impact of anisotropy fields and demagnetizing fields on the precession frequency is taken into account [53]:

$$
\omega=\gamma \sqrt{\left(H_{e x}+H_{K}\right)\left(H_{e x}+H_{K}+\mu_{0} M_{e f f}\right)}
$$

$H_{k}$ is the magnetic crystalline anisotropy field, and $M_{e f f}$ is the effective magnetization which is the saturation magnetization reduced by surface anisotropy. Parameters $\gamma, H_{k}$ and $M_{\text {eff }}$ can be obtained by fitting the dependence of $\omega$ on $H_{e x}$. The precession is eventually relaxed due to the damping torque. However, a forced ferromagnetic resonance happens when a RF magnetic field with a frequency same as magnetization precession frequency is applied to the system. With the RF field frequency fixed, an absorption line can be detected during a $H_{\text {ex }}$ sweep, indicating a ferromagnetic resonance with which the energy from the RF field is absorbed by the system most effectively. The linewidth $\left(\Delta H_{e x}\right)$ due to the magnetization relaxation consists of two terms,

$$
\Delta H_{e x}=\Delta H_{0}+\frac{2 \alpha}{\sqrt{3} \gamma^{\prime}} w
$$

The first term is the $\Delta H_{0}$ relaxation that is complicated and not necessarily a constant. One of the examples is the two magnon scattering process. It is the lowest order magnon coupling process which relaxes the precession by redistributing energy within the magnetic system $[35,37,54]$. The second term arises from the intrinsic Gilbert damping 
( $\alpha$ ) that transforms energy to a thermal bath of phonons or conducting electrons. In the case of a constant $\Delta H_{0}$, the Gilbert damping parameter can be obtained from the slope of a linear fitting of $\omega$ verse $\Delta H_{e x}$.

The FMR measurements for this thesis work were performed by T. Mewes’ group at University of Alabama. The FMR spectra were obtained by a broadband coplanar waveguide field-swept FMR setup, with a frequency limit up to $50 \mathrm{GHz}$. The details of the setup can be found in Ref.[54]. 


\subsubsection{X-ray diffraction/Surface Roughness}

The crystal structures in this work are characterized using X-ray diffraction techniques. Using Bragg's law, one can determine the crystal structure and lattice parameters according to the diffraction angles of the Bragg peaks, or predict the positions of the Bragg peaks with knowledge of the crystal structure.

A Rigaku Smart lab XRD system performed all the structure characterizations of this work. The X-ray source is operated at an accelerating voltage of $44 \mathrm{keV}$ and $40 \mathrm{Amps}$. $\mathrm{Cu} \mathrm{K \alpha}$ radiation is used with a wavelength $\sim 1.541 \AA$. A Ge(2×220) mirror monochromator was used for high resolution $\mathrm{X}$-ray experiments.

The surface roughness is determined by Atomic force microscopy (AFM). As the silicon tip on a cantilever approaches the proximity of the sample surface, the force between the tip and the sample bends the cantilever. The surface morphology is thus determined by detecting the laser deflection reflected from the cantilever surface. 


\section{Cr-doped CoFeB films}

\subsection{Introduction}

As discussed in Chapter 2, magnetic tunnel junctions (MTJs) utilizing MgO tunnel barrier are the subject of intense research due to the large tunneling magnetoresistance (TMR) effect [51,52], which has potential application as memory elements in spin-transfer-torque magnetic random access memory (STT-MRAM) [53,54,55]. It is now generally accepted that TMR is very sensitive to the electronic structures of the electrodes and the tunnel barrier, and to the interfacial bonding between them. Recently, ferromagnetic CoFe alloys made amorphous by the addition of boron have become of special interest because MTJs incorporating them show the highest TMR values at room temperature compared with other magnetic electrodes. This was reported for sputtered $\mathrm{CoFeB} / \mathrm{MgO} / \mathrm{CoFeB}$ MTJ with TMR ratios of up to $500 \%$ at room temperature (RT) and $1010 \%$ at $5 \mathrm{~K}$, a low switching current of $49 \mu \mathrm{A}$ [56]. An asdeposited $\mathrm{CoFeB}$ layer exhibits an amorphous structure, and MgO barrier layer grown on the amorphous CoFeB is a (001) textured polycrystalline film. By thermal annealing, the (001) oriented body-centered cubic (BCC)-FeCo is crystallized while maintaining perfect coherency with the MgO barrier, which is required for coherent tunneling across the MgO barrier and proven to be the best way to obtain large TMR $[57,58]$. However, a large damping constant of 0.01 and a high magnetization of $1200 \mathrm{emu} / \mathrm{cc}$ of $\mathrm{CoFeB}$ free layer were reported [59], resulting in the difficulty of the reduction in the intrinsic critical current density $\left(J_{c}\right)$ of current-induced magnetization switching.

Ferromagnetic films with low magnetization and high spin polarization are promising spintronic materials for the further reduction in $J_{\mathrm{c}}[59,60]$. Due to the amorphous nature, CoFeB alloys are highly insensitive to the miscibility of their constituents, allowing their 
magnetization to be controlled by adjusting the transition-metal concentration or substituting iron/cobalt with chromium/vanadium in the alloy. Cr metal exhibits a BCC structure, which would not affect the crystal structure of the free layer after annealing. Incorporating Cr into CoFeB was found effectively to reduce the switching current [60]. The reduction in $J_{\mathrm{c}}$ by a factor of four to $4.9 \times 10^{5} \mathrm{~A} / \mathrm{cm}^{2}$ was achieved by using a $\mathrm{Co}_{40} \mathrm{Fe}_{32.7} \mathrm{Cr}_{7.3} \mathrm{~B}_{20}$ free layer while maintaining the magnetoresistance (MR) ratio of around $60 \%$ [61]. However, upon the further increase in Cr, a significant reduction in MR was observed in MTJs with free layer of $\left(\mathrm{Co}_{75} \mathrm{Fe}_{25}\right)_{80-x} \mathrm{Cr}_{x} \mathrm{~B}_{20}$ [62]. A high $\mathrm{B}$ content in the electrode is necessary to obtain a fully amorphous structure in the as-deposited state. However, by considering the limited solubility of B in the BCC phase, such high boron content ( 20 at. \%) in the CoFeCrB electrode raises a probability of a secondary boride phase existing after thermal annealing, which may be the cause for the degradation of TMR in high Cr compositions.

In this study, we fabricated amorphous CoFeCrB ribbons for a wide range of Cr contents and explored the influence of Boron content to the crystallization process with increasing $\mathrm{Cr}$ substitution. The specific compositions were chosen in order to achieve a uniform crystalline structure with precipitation of only BCC phase, making the materials suitable for device application. The structural and magnetic properties of CoFeCrB thin films depending on the annealing treatment and Cr concentration were also investigated, the findings are presented herein.

\subsection{EXPERIMENT}

Ingots with compositions of $\mathrm{Co}_{40} \mathrm{Fe}_{\mathrm{x}} \mathrm{Cr}_{\mathrm{y}} \mathrm{B}_{\mathrm{z}}(\mathrm{x}+\mathrm{y}+\mathrm{z}=60$ and $\mathrm{y}=0-30)$ were prepared by arc melting the mixture of high purity Fe, Co, Cr, and B metals in a high purity argon atmosphere, each ingot was re-melted four times to ensure the chemical homogeneity. 
Amorphous ribbons, typically about $1.25 \mathrm{~mm}$ wide and $25 \mu \mathrm{m}$ thick, were produced from the ingots melted spun on a water-cooled copper hearth in a high purity argon atmosphere. Chemical compositions of the ribbons were checked by inductively coupled plasma-mass spectroscopy and no detectable deviation from the nominal composition was observed. Crystallization behaviors were monitored by differential scanning calorimeter (DSC) at a constant heating rate of $5{ }^{\circ} \mathrm{C} / \mathrm{min}$ under purified argon atmosphere in the temperature range from $50{ }^{\circ} \mathrm{C}$ to $600{ }^{\circ} \mathrm{C}$. As-spun ribbons were isochronally annealed for $1 \mathrm{~h}$ in a tubular furnace by encapsulation in evacuated quartz tubes at various temperatures.

CoFeCrB films were prepared and characterized by our collaborators Y. Cui and J.W. Lu in University of Virginia. Films were deposited on thermally oxidized silicon substrates by $r f$ magnetron sputtering at ambient temperature. Two targets with different stoichiometric compositions $\left(\mathrm{Co}_{43} \mathrm{Fe}_{29} \mathrm{Cr}_{10} \mathrm{~B}_{18}\right.$ and $\left.\mathrm{Co}_{40} \mathrm{Fe}_{18} \mathrm{Cr}_{30} \mathrm{~B}_{12}\right)$ were sputtered concurrently with different power ratios so that specific compositions can be explored. All the film samples were in a structure of $\mathrm{Si} / \mathrm{SiO}_{2} / \mathrm{Ta}(7 \mathrm{~nm}) / \mathrm{CoFeCrB}(30 \mathrm{~nm}) / \mathrm{Ta}(7 \mathrm{~nm})$. The Ta film was deposited as buffering layer to improve the surface conditions before $\mathrm{CoFeCrB}$ deposition and as capping layer to protect $\mathrm{CoFeCrB}$ layer from oxidation. Subsequently, the films were annealed at temperatures $\left(T_{\mathrm{a}}\right)$ ranging from $350{ }^{\circ} \mathrm{C}$ to $450{ }^{\circ} \mathrm{C}$ in vacuum with an in-plane magnetic field of 1400 Oe for one hour.

\subsection{RESULTS AND DISCUSSION}

\subsubsection{Ribbons}

The amorphous structures of as-spun ribbons were confirmed by XRD and TEM observations. A typical example of as-spun $\mathrm{Co}_{40} \mathrm{Fe}_{32} \mathrm{Cr}_{10} \mathrm{~B}_{18}$ ribbon is shown in Fig. 4.1. The broad band in x-ray spectrum ( $\theta-2 \theta$ profile) indicated a fully amorphous feature due to the 
extremely high cooling speed, as performed in Fig. 4.1(a). The bright-field TEM image in Fig. 4.1(b) shows an amorphous matrix without crystallization nuclei, together with a halo ring as shown in the inset electron diffraction pattern. The TEM results were consistent with the XRD, indicating all the as-spun ribbons were amorphous in nature, without the formation of a longrange structural order.
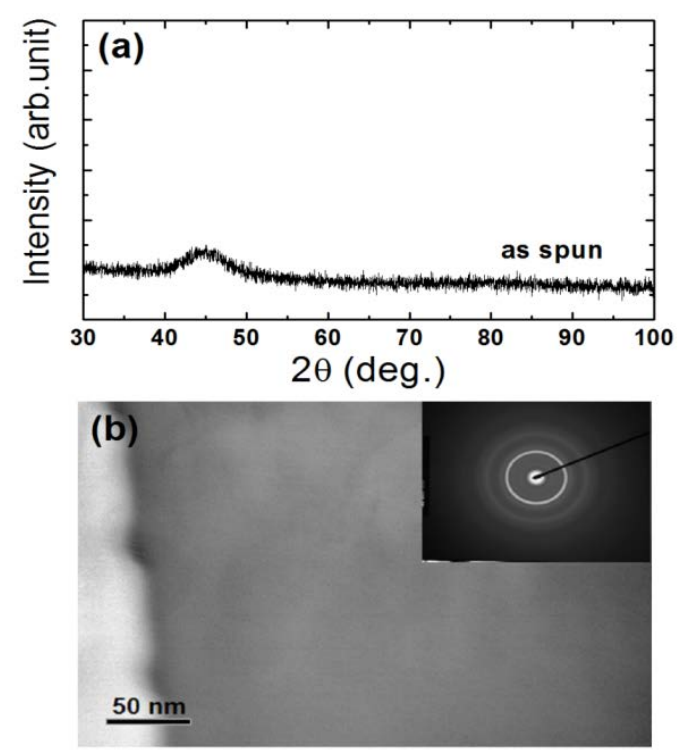

Figure 4.1. (a) XRD pattern and (b) Bright-field TEM image of as-spun $\mathrm{Co}_{40} \mathrm{Fe}_{32} \mathrm{Cr}_{10} \mathrm{~B}_{18}$ ribbon; The inset of Fig. (b) shows the selected area diffraction pattern.

Figure 4.2(a) shows continuous DSC curves of as-spun ribbons for two alloys with $\{x, y, z\}=\{32,10,18\}$ and $\{30,10,20\}$. For comparison, the curve of the as-spun $\mathrm{Co}_{40} \mathrm{Fe}_{40} \mathrm{~B}_{20}$ ribbon was also included in the same figure. The onset temperature of crystallization, $T_{x}$, was determined from the intersection of straight lines tangent to the DSC curve at the beginning of the exothermic peak. For as-spun ribbon of $\mathrm{Co}_{40} \mathrm{Fe}_{32} \mathrm{Cr}_{10} \mathrm{~B}_{18}$, two separate distinct exothermic peaks can be seen upon heating. The samples were heated at different temperatures to identify the crystallization sequence, which was clarified by XRD patterns of annealed $\mathrm{Co}_{40} \mathrm{Fe}_{32} \mathrm{Cr}_{10} \mathrm{~B}_{18}$ 
ribbons as shown in Fig. 4.2(b). When the ribbon was heated at $418{ }^{\circ} \mathrm{C}$ (temperature corresponds to $\mathrm{T}_{\mathrm{x} 1}$ ) all diffraction peaks, at $2 \theta$ values of $44.95^{\circ}, 65.56^{\circ}$ and $82.94^{\circ}$, can be indexed well to a single BCC phase. However, heating the sample to a higher temperature of $508{ }^{\circ} \mathrm{C}$ (temperature corresponds to $\mathrm{T}_{\mathrm{x} 2}$ ) resulted in the appearance of a large number of peaks, which could be indexed to different boride-type phases such as $(\mathrm{FeCo})_{3} \mathrm{~B}$, $(\mathrm{FeCo})_{2} \mathrm{~B}$ and $(\mathrm{FeCo})_{23} \mathrm{~B}_{6}[63]$. These results indicated the existence of two-step crystallization reaction corresponding to two separate exothermic peaks in DSC curve of as-spun $\mathrm{Co}_{40} \mathrm{Fe}_{32} \mathrm{Cr}_{10} \mathrm{~B}_{18}$ ribbon. The primary exothermic peak was attributed to $\alpha$-BCC crystals followed by a secondary peak with the precipitation of additional boride phases from the amorphous state. The as-spun $\mathrm{Co}_{40} \mathrm{Fe}_{40} \mathrm{~B}_{20}$ ribbon showed similar crystallization behavior.
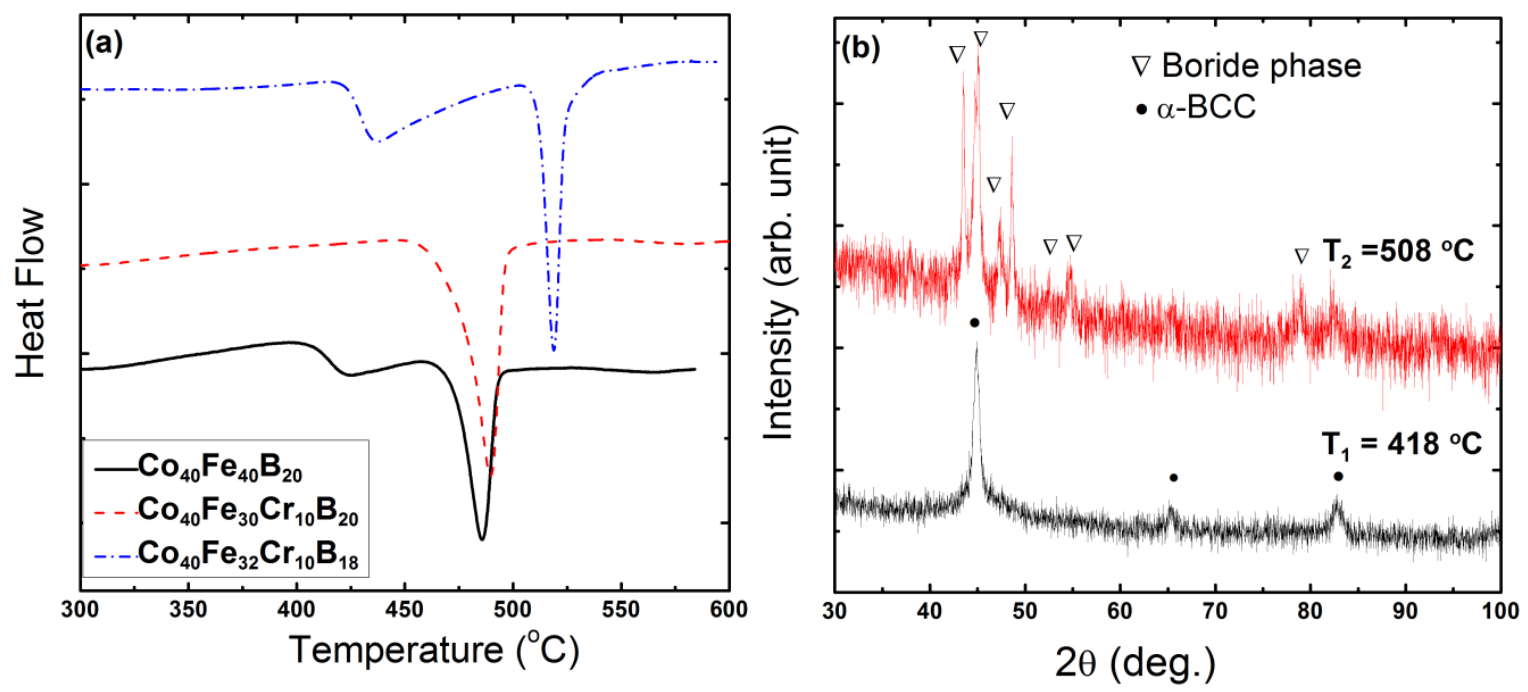

Figure 4.2 (a) DSC curves of as-spun $\mathrm{Co}_{40} \mathrm{Fe}_{x} \mathrm{Cr}_{y} \mathrm{~B}_{z}$ ribbons with $y=0,\{x, z\}=\{40,20\}$ and $y=10,\{x$, $z\}=\{30,20\}$, \{32,18\}. (b) XRD patterns of $\mathrm{Co}_{40} \mathrm{Fe}_{32} \mathrm{Cr}_{10} \mathrm{~B}_{18}$ ribbons after annealing up to the onset of second crystallization stage.

According to DSC curves (Fig. 4.2(a)), the temperature difference between the two crystallization phases was $\sim 62{ }^{\circ} \mathrm{C}$ for $\mathrm{Co}_{40} \mathrm{Fe}_{40} \mathrm{~B}_{20}$ ribbon. With $\mathrm{Cr}$ substitution of $10 \%$ the two 
peaks initially superposed for the composition with $20 \%$ boron, the crystallization temperature of $\alpha$-BCC was a little lower than that of boride in $\mathrm{Co}_{40} \mathrm{Fe}_{30} \mathrm{Cr}_{10} \mathrm{~B}_{20}$ ribbon and caused the overlapping of the two crystallization signals. However, this temperature difference increased gradually with reduced B content (Fig. 4.2(a)) to $\sim 90{ }^{\circ} \mathrm{C}$ for B of $18 \%$. These can be attributed to a stronger role of antiferromagnetic coupling at high $\mathrm{Cr}$ content, which also tended to increase the crystallization temperature of BCC phase, as shown in the Table I. Apparently in order to have two separated crystalline processes, there is a necessary to reduce B content accompanied with increasing $\mathrm{Cr}$ substitution.

In the study we chose four $\mathrm{Co}_{40} \mathrm{Fe}_{\mathrm{x}} \mathrm{Cr}_{\mathrm{y}} \mathrm{B}_{\mathrm{z}}$ compositions for thin film depositions, with combinations of $\{\mathrm{x}, \mathrm{y}, \mathrm{z}\}$ identified in Table I. Notably, the onset crystallization temperature $\left(\mathrm{T}_{\mathrm{x} 1}\right)$ of BCC phase increased from $418^{\circ} \mathrm{C}$ at $\mathrm{Cr}$ of $10 \%$ to $456^{\circ} \mathrm{C}$ at $\mathrm{Cr}$ of $30 \%$, indicating that BCC phase formed at a higher temperature with increasing $\mathrm{Cr}$ substitution. The magnetization decreased precipitously with increasing $\mathrm{Cr}$ substitution and was finally below $100 \mathrm{emu} / \mathrm{cc}$ at $\mathrm{Cr}$ of $30 \%$, within the expectation of the antiferromagnetism of Cr.

Table 4.1 Summary of the onset temperatures $\left(T_{x}\right)$ of crystallization phases determined by DSC measurements, saturation magnetization $\left(M_{s}\right)$ for as-spun $\mathrm{Co}_{40} \mathrm{Fe}_{\mathrm{x}} \mathrm{Cr}_{\mathrm{y}} \mathrm{B}_{\mathrm{z}}$ alloys.

\begin{tabular}{|l|l|l|l|l|l|l|}
\hline Composition & $x$ (at. \%) & $y$ (at. \%) & $z$ (at. \%) & $T_{x 1}\left({ }^{\circ} \mathrm{C}\right)$ & $\left.T_{x 2}{ }^{\circ} \mathrm{C}\right)$ & $M_{s}(\mathrm{emu} / \mathrm{cc})$ \\
\hline $\mathrm{Co}_{40} \mathrm{Fe}_{32} \mathrm{Cr}_{10} \mathrm{~B}_{18}$ & 32 & 10 & 18 & 418 & 508 & 912 \\
\hline $\mathrm{Co}_{40} \mathrm{Fe}_{27} \mathrm{Cr}_{16} \mathrm{~B}_{17}$ & 27 & 16 & 17 & 426 & 541 & 530 \\
\hline $\mathrm{Co}_{40} \mathrm{Fe}_{24} \mathrm{Cr}_{22} \mathrm{~B}_{14}$ & 24 & 22 & 14 & 437 & 560 & 206 \\
\hline $\mathrm{Co}_{40} \mathrm{Fe}_{20} \mathrm{Cr}_{30} \mathrm{~B}_{10}$ & 20 & 30 & 10 & 456 & 573 & 83 \\
\hline
\end{tabular}


On the other hand, the crystallization temperature for the boride phases was decreased with an increasing B content. This indicated that with a high B content, the limited solubility of B in the BCC phase saturated at a relatively low temperature, and thus promoted the Boron Segregation. In general a reduced B content is required when increasing the $\mathrm{Cr}$ content, to avoid the overlapping of the onset crystallization temperatures for the BCC and boride phases.

\subsubsection{Thin films (contributed by Y.Cui from University of Virginia)}

According to the composition engineering in the ribbon materials, the $\mathrm{Cr}$ and $\mathrm{B}$ contents were selected carefully in the co-sputtered targets for thin film depositions to achieve BCC phase with Boride phase suppressed. A typical crystallization process similar to the ribbon materials was demonstrated in Fig. 4.3. $2 \theta$ scans were taken in $\mathrm{Co}_{40} \mathrm{Fe}_{20} \mathrm{Cr}_{30} \mathrm{~B}_{10}$ films annealed at temperatures ranging from $350^{\circ} \mathrm{C}$ to $450^{\circ} \mathrm{C}$. The films remained amorphous /nano-crystallized as the annealing temperature was below $400^{\circ} \mathrm{C}$. Peak (110) at around $44.94^{\circ}$ appeared with an annealing temperature $\sim 400^{\circ} \mathrm{C}$, indicating the $\alpha$-BCC structure in thin films highly textured in (110) orientation. As the annealing temperature rose up to $450^{\circ} \mathrm{C}$, an extra peak corresponding to a boride-type phase was detected around $42.9^{\circ}$, consistent with the observation in ribbon materials. The (110)-BCC peak did not shift much with varied compositions. Using Bragg's law,

the lattice constant a $\sim 2.86 \AA$ can be determined, which is nearly equal to the reported value. ${ }^{12}$ The misfit (3.9\%) between $\mathrm{Co}_{40} \mathrm{Fe}_{20} \mathrm{Cr}_{30} \mathrm{~B}_{10}$ and $\mathrm{MgO}$ barrier in this orientation is small, which is comparable to $\mathrm{CoFeB} / \mathrm{MgO}$. An epitaxial growth of $\mathrm{CoFeCrB}$ on $\mathrm{MgO}$ layer is thus expected resulting a high coherent tunneling when incorporated into a MTJ. 


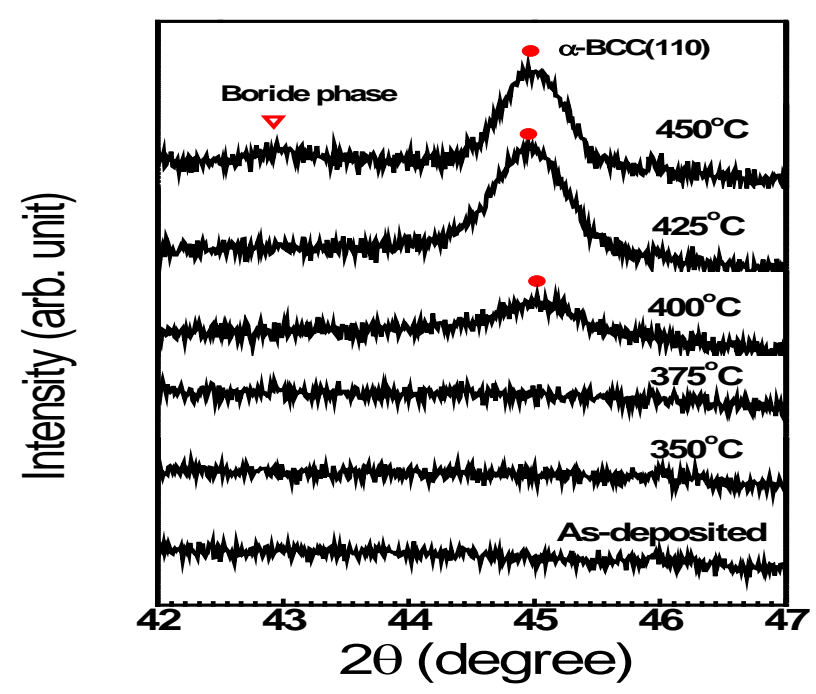

Figure 4.3. $X R D \theta-2 \theta$ scans of $\mathrm{Co}_{40} \mathrm{Fe}_{20} \mathrm{Cr}_{30} \mathrm{~B}_{10}(30 \mathrm{~nm})$ films annealed at temperatures ranging from $350^{\circ} \mathrm{C}$ to $450^{\circ} \mathrm{C}$. Courtesy of Yishen Cui, et al from University of Virginia.

By varying the growth rate of each target, the Cr content in thin films was systematically tuned from $10 \%$ to $30 \%$. Fig. 4.4 showed the impact from Cr content on the magnetizations and coercive fields for both as-deposited and annealed films with annealing treatments with temperature ranging from $350{ }^{\circ} \mathrm{C}$ to $450{ }^{\circ} \mathrm{C}$. In the films with $\mathrm{Cr}$ of $10 \%$, the magnetization was 651emu/cc in as-deposited state and increased to 850 emu/cc after annealing, which were much lower than the typical value obtained in $\mathrm{CoFeB}$. The magnetization was further reduced with an increasing $\mathrm{Cr}$ content, consistent with previous reports. As the $\mathrm{Cr}$ content increased to 30\%, the magnetization was close to 0 in as-deposited state and increased to 300 emu/cc after annealing treatments. These were within expectation giving the antiferromagnetism of Cr. The rapid reduction of magnetization made $\mathrm{CoFeCrB}$ as a prospective candidate for the free layer in STTMRAM. 
It is also worth pointing out that both onset crystallization temperatures of BCC phase $\left(T_{x 1}\right)$ and saturation magnetizations were shifted to a lower level in thin film samples compared with the ribbon samples with the same composition.

In addition, the saturation magnetization of $\mathrm{CoFeCrB}$ films increased with increasing annealing temperature, as performed in Fig. 4.4(a), which can be attributed to the boron diffusion [63-65]. During high temperature treatment, boron atoms might diffuse away from CoFeCrB at interfaces, resulting in the reduction of magnetic impurities (boron atoms) and therefore increase the magnetic moment. Since the size of the boron atom is much smaller than the other atoms (Co, Fe, Cr), boron diffusion must be easier and faster than other atoms' diffusion, and therefore dominates the magnetization variation during annealing. To understand the boron behavior as a function of annealing temperature, further investigations with high-resolution transmission electron microscopy would be necessary.
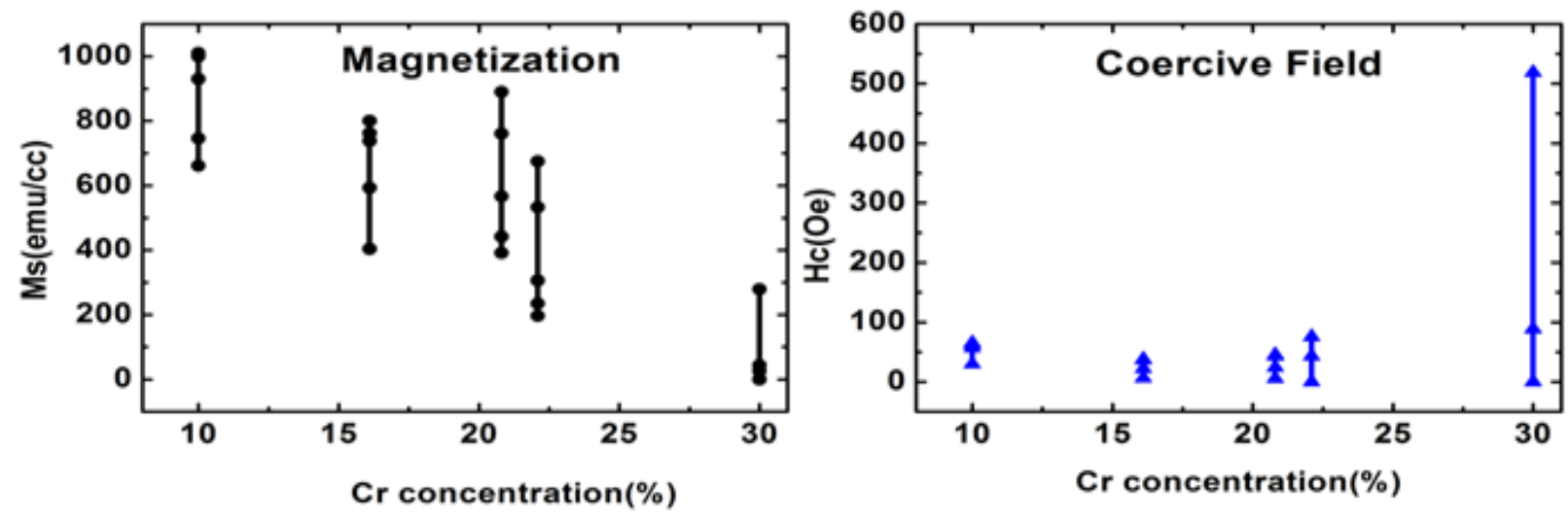

Figure 4.4 The impact from Cr content on the (a) saturation magnetizations and (b) coercive fields for both as-deposited and annealed films with annealing treatments at various temperatures.

It was also demonstrated in Fig. 4.4(b) that the coercive field was enhanced with increasing $\mathrm{Cr}$ content. With $\mathrm{Cr}$ content $\sim 30 \%$, the coercive field was increased up to 500 Oe 
after annealing to BCC phase. The coercivity enhancement has not been reported in Co-Fe-Cr-B system before. But it is worth noting that large coercive field was observed in Co-Fe-Cr system in previous reports [66-68]. The Kaneko alloy Co-Fe-Cr with $\mathrm{Cr}$ ranged from 20\% to $40 \%$ has a spinodal decomposition from a $\alpha$-BCC solid solution into a two-phase structure in which the Fe rich $\alpha_{1}$-phase clusters were magnetically isolated in $\mathrm{Cr}$ rich $\alpha_{2}$-phase matrix. The high coercive field could be ascribed to the domain wall pinning effect or incoherent rotation process among $\alpha_{1}$-phase clusters [66]. This mechanism could be similarly applied to Co-Fe-Cr-B system.

\subsubsection{Damping parameter characterization}

The Gilbert damping parameter was characterized for $\mathrm{Co}_{43} \mathrm{Fe}_{29} \mathrm{Cr}_{10} \mathrm{~B}_{18}$ films. In asdeposited samples the damping parameter was $\sim 0.085$, and it was reduced to 0.006 after annealing at 450 degree (no boride phase was detected at this temperature). The value was comparable with the value obtained in $\mathrm{Co}_{40} \mathrm{Fe}_{20} \mathrm{~B}_{20}$ films [57]. Besides, it was much lower than previously reported values (0.02) for $\mathrm{Co}_{40} \mathrm{Fe}_{40}-\mathrm{XCr}_{\mathrm{x}} \mathrm{B}_{20}$ films [59]. It was also indicated in reference [59] that the damping parameter remained as a constant regardless of the change of the Cr content $x$ in the range of 0 to $\sim 18 \%$.

\subsection{Summary}

A two-stage crystallization was observed in $\mathrm{CoFeCrB}$ ribbons. A bcc phase was formed at a relatively low annealing temperature followed by boron segregation at a higher annealing temperature. The onset temperature for the bcc phase was raised with a higher $\mathrm{Cr}$ content. Besides, as the Boron content was increased, the limited solubility of B in the bcc phase saturated a lower annealing temperature, leading to a lower onset temperature for the boride phase. A careful control of the composition and annealing temperature is thus required to achieve a pure $\alpha$-bcc phase in CoFeCrB. 
A similar crystallization process was observed in CoFeCrB thin films. The magnetic behaviors of $\mathrm{CoFeCrB}$ thin films were characterized as a function of the Cr content. The magnetization was significantly reduced as the $\mathrm{Cr}$ content increased in the films, also $\mathrm{Cr}$ substitution largerly enhanced the coercive field of CoFeB. This could be ascribed to the spinodal decomposition of Fe-rich $\alpha_{1}$ phase clusters from the Cr-rich $\alpha_{2}$-phase matrix. The domain wall- $\alpha_{1}$ particle interaction results in a high coercive field. The Gilbert damping parameter was estimated around 0.006 in $\mathrm{Co}_{43} \mathrm{Fe}_{29} \mathrm{Cr}_{10} \mathrm{~B}_{18}$ films, which is comparable with $\mathrm{Co}_{40} \mathrm{Fe}_{20} \mathrm{~B}_{20}$ films. 


\section{Interfacial perpendicular magnetic anisotropy}

\subsection{Introduction}

Recently, strong interfacial perpendicular anisotropy between $\mathrm{CoFeB}$ and MgO, especially in Fe-rich composition [75], was shown with a high tunnel magnetoresistance ratio over $120 \%$, high thermal stability at dimension as low as $40 \mathrm{~nm}$ diameter and a low switching current of 49 $\mu \mathrm{A}$ [76]. However, large damping constant $(\alpha)$ of 0.01 was reported [79], resulting in the difficulty of the reduction in the intrinsic critical current density $\left(J_{\mathrm{c} 0}\right)$ of low current-induced magnetization switching.

PMA alloy films with high spin polarization and low damping constant are promising spintronic materials for the further reduction in $J_{\mathrm{c} 0}$ [77]. Incorporating Ge into CoFe is found to increase the spin polarization in the bulk electron transport. The maximum polarization is achieved in the Ge composition range of $20 \%-30 \%$, which is in concert with the damping minimum in $\left(\mathrm{Co}_{50} \mathrm{Fe}_{50}\right)_{100-x} \mathrm{Ge}_{x}$ films [78]. The low damping constant of $\alpha \sim 0.0025$ is significantly smaller than 0.01 of $\mathrm{CoFeB}$ [79]. In this letter, we fabricated Fe-rich $\mathrm{Co}_{20} \mathrm{Fe}_{50} \mathrm{Ge}_{30}$ (CoFeGe) films faced to MgO layer and demonstrated the perpendicular magnetization of CoFeGe thin films. Another advantage of this composition is that Fe-rich composition has stronger interfacial anisotropy, which is consistent with theoretical calculations that BCC $\mathrm{Fe}(001) / \mathrm{MgO}(001)$ interface anisotropy is greater than that of $\mathrm{BCC} \mathrm{Co}(001) / \mathrm{MgO}(001)$.

\subsection{Experiments}

CoFeGe films in this study were prepared on single crystalline MgO (001) substrates by RF magnetron sputtering system. The base pressure of the sputtering chamber was below $5 \times 10^{-7}$ Torr. Cubic MgO (001) substrates were selected to provide a seeding effect (the lattic mismatch between $\mathrm{MgO}$ and $\mathrm{CoFeGe}$ is 3.0\%). Before the deposition, all the MgO substrates were 
annealed at $150{ }^{\circ} \mathrm{C}$ for 30 min in situ to remove the surface contamination such as $\mathrm{H}_{2} \mathrm{O}$ for a better seeding condition. Subsequently, the CoFeGe alloy films were deposited by means of cosputtering with the elemental targets under a processing pressure around $4 \times 10^{-3}$ Torr. The capping MgO layer was formed directly from a sintered $\mathrm{MgO}$ target to protect CoFeGe layer from oxidation. All the samples deposited at room temperature have typical structure consisting of $\mathrm{MgO}(001) / \mathrm{CoFeGe}(t) / \mathrm{MgO}(5 \mathrm{~nm})$ ( $t$ is the thickness of CoFeGe layer ). Subsequently, the stacks were annealed at a temperature $T_{\mathrm{a}}$ of $250{ }^{\circ} \mathrm{C}$ in a vacuum under a perpendicular magnetic field of 1400 Oe for two hours.

\subsection{Results and Discussion}

To investigate the structure of CoFeGe films, $\mathrm{x}$-ray spectrum $(\theta-2 \theta$ profile) is shown in Fig. 5.1 (a), which was obtained from the stack with $\mathrm{CoFeGe}(20 \mathrm{~nm})$ after annealing. The strongest peak at about $42.9^{\circ}$ is due to the diffraction of (002) MgO planes. Two small diffraction peaks were observed around $31.17^{\circ}$ and $65.01^{\circ}$ in the $2 \theta$ scan, which correspond to (002) and (004) CoFeGe planes. This indicates that the fabricated CoFeGe film has a (001)-oriented B2 order, characterized by total disorder between $\mathrm{Fe}$ and $\mathrm{Ge}$, while Co atoms occupy regular site [78]. The in-plane epitaxial relationship between the CoFeGe film and $\mathrm{MgO}$ was determined by the $\Phi$ scan of the reflection from the (022) planes of CoFeGe and MgO. The results are displayed in Fig. 5.1(b). The four CoFeGe (022) peaks are due to the four-fold symmetry along the c-axis of CoFeGe, which also applied to the MgO (022) peaks. A separation of $45^{\circ}$ from the positions of (022) peaks for the CoFeGe film to those of (022) peaks for the MgO substrate, confirms their epitaxial relationship to be $\mathrm{CoFeGe}(001)[100] / / \mathrm{MgO}(001)[110]$. The B2-ordered $\mathrm{Co}_{20} \mathrm{Fe}_{50} \mathrm{Ge}_{30}$ is not half metallic but has a perfectly spin-polarized $\Delta_{1}$ band along the [001] direction; thus, 
significant tunnel magneto-resistance (TMR) ratio enhancement attributed to the coherent tunneling effect is expected in CoFeGe-based MTJs. Using Bragg's law, the lattice constant a $5.736 \AA$ can be determined, which is nearly equal to the reported value [80]. The misfit (-3.8\%) between $\mathrm{CoFeGe}$ and $\mathrm{MgO}$ in this orientation enables the fabrication of a high-quality CoFeGe/MgO(001) epitaxial structure for low-resistance-area (RA) magnetic tunnel junctions (MTJs), which are essential to the observation of spin-transfer switching. To investigate the microstructure of CoFeGe film, cross-sectional HRTEM images were taken from the annealed stack with CoFeGe(2nm). To identify the phases, we measured the $d$ spacing from the fast Fourier transformation (FFT) of the HRTEM image. We cannot unambiguously identify the (002) texture. However, CoFeGe (110) planes are evident, indicating a body centered cubic (bcc) symmetry in thin films.
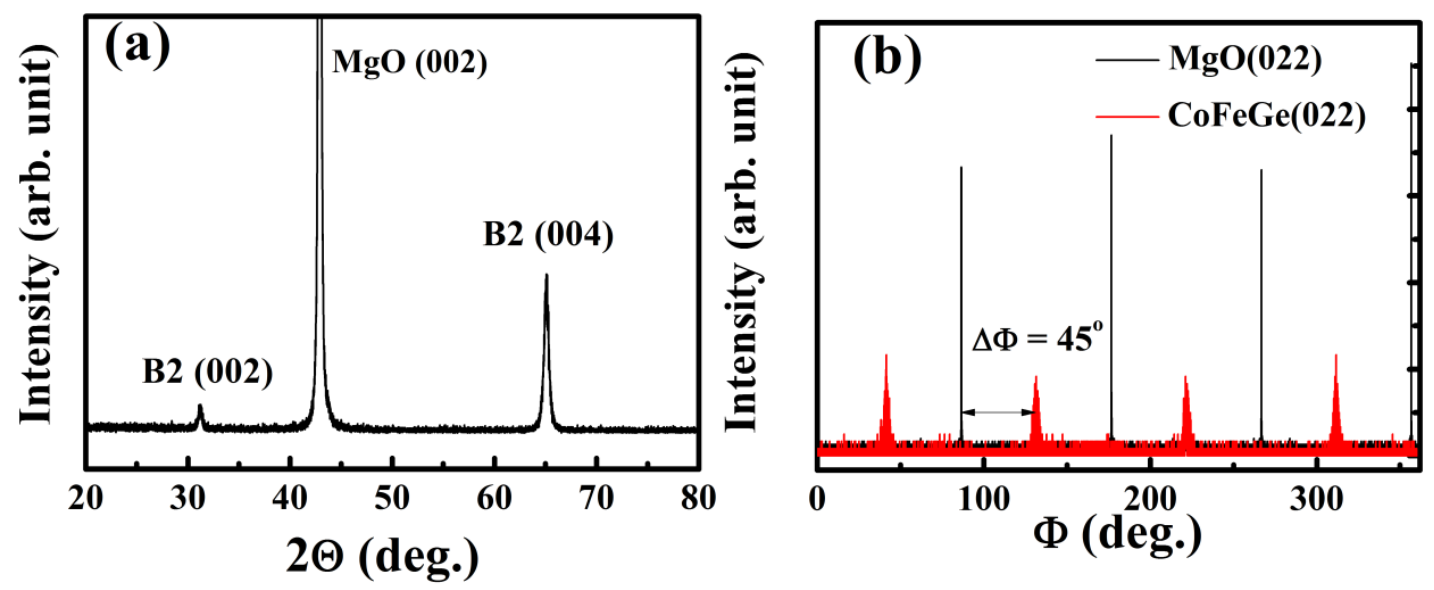

Figure 5.1 (a) XRD $\theta$-2 2 scan of $\mathrm{Co}_{20} \mathrm{Fe}_{50} \mathrm{Ge}_{30}(20 \mathrm{~nm})$ film on $\mathrm{MgO}$ (001). (b) $\Phi$ scan on the $\mathrm{Co}_{20} \mathrm{Fe}_{50} \mathrm{Ge}_{30}(20 \mathrm{~nm})$ sample. The red line represents data taken at $2 \theta$ of $44.66^{\circ}$ (CoFeGe (022) peak) and the black one represents data taken at $62.45^{\circ}$ (MgO (022) peak).

It has been reported that for conventional PMA material, a high annealing treatment of over $400{ }^{\circ} \mathrm{C}$ is required to obtain a highly ordered structure[81]. However, the average interface roughness increases sharply when the annealing temperature is increased to over $400{ }^{\circ} \mathrm{C}$. This 
might be a hindrance in achieving a large tunneling spin polarization. In contrast, our investigated CoFeGe films only require an annealing temperature of $250^{\circ} \mathrm{C}$ to form a highly spinpolarized chemically B2-ordered structure, which performs as a promising candidate for spintronics devices.

The magnetic behaviors of CoFeGe films were characterized along MgO [100] and [001] directions using the VSM option in Quantum Design VersaLab. The interfacial perpendicular anisotropy in thin CoFeGe films appeared after annealing, whereas magnetic easy axis was inplane before annealing for all thicknesses, which may be due to the improvement of crystallization of the CoFeGe layer with optimized interface. Figure 5.2 shows the typical inplane and out-of-plane hysteresis loops of the magnetization for the annealed stacks of $\operatorname{MgO}(001) / \operatorname{CoFeGe}(t) / \mathrm{MgO}(5 \mathrm{~nm})$ with nominal thickness $t=1.2 \mathrm{~nm}$ and $2 \mathrm{~nm}$, respectively. The sample with $t_{\mathrm{CoFeGe}}=2 \mathrm{~nm}$ has an easy in-plane axis. A clear perpendicular anisotropy was realized with the in-plane saturation field $H_{k}=5500$ Oe and out-of-plane coercivity $H_{c}=10$ Oe for $t=1.2 \mathrm{~nm}$. The saturation magnetization is $903 \mathrm{emu} / \mathrm{cc}$, which is about half of that in conventional CoFeB system[79]. The large anisotropy field shown here allows the use of thinner magnetic layers and hence faster switching speeds, compared to in-plane devices, for the same activation energy. The total perpendicular anisotropy energy density $K$ at this thickness, which determines the thermal stability, is $1.2 \times 10^{6} \mathrm{erg} / \mathrm{cm}^{3}$, as calculated by evaluating the area enclosed between the in-plane and perpendicular $\mathrm{M}-\mathrm{H}$ curves [81]. This method was acceptable for a semiquantitative comparison given the isothermal measurement conditions, at which the sample was near the equilibrium state. The squareness of out-of-plane hysteresis loop, however, is not perfect. 

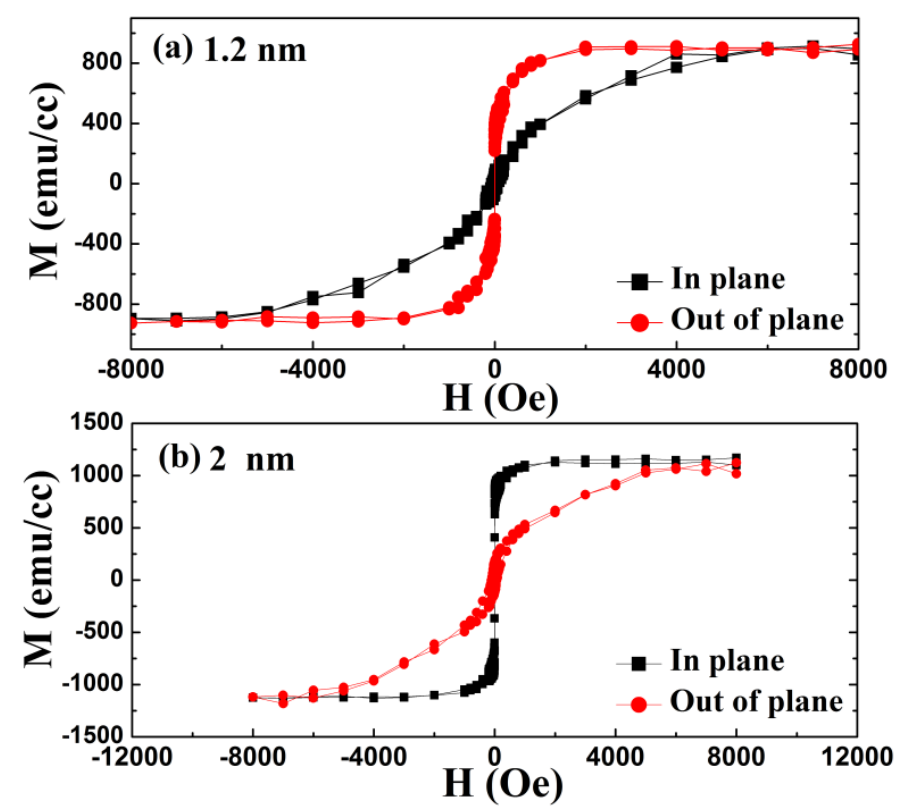

Figure 5.2 In-plane (black) and out-of-plane (red) magnetic hysteresis loops for (a) $1.2 \mathrm{~nm}$ and (b) $2 \mathrm{~nm}$ annealed $\mathrm{Co}_{20} \mathrm{Fe}_{50} \mathrm{Ge}_{30}$ films, respectively.

It was also noticed that the magnetization $M_{s}$ of the film was reduced as the thickness was reduced (Fig. 5.3 circles). Such a trend implied the existence of a dead layer, i.e., the deteriorated crystalline structure or an intermixing between CoFeGe and the MgO layer. To determine the thickness of the dead layer and extract the saturation magnetization $M_{s}$ for CoFeGe in this series of samples, we linearly fitted the magnetization per unit area, $m_{s}=M_{s} t_{\mathrm{CoFeGe}}$, as the film thickness ranged from $1.0 \mathrm{~nm}$ to $2.5 \mathrm{~nm}$ (Fig. 5.3 triangles). The $\mathrm{x}$-intercept is the dead layer thickness, which was found to be $0.4 \mathrm{~nm}$, while the slope gives the extracted $M_{s}$ of $1250 \mathrm{emu} / \mathrm{cc}$. This value is consistent with the experimental bulk magnetization. It is not clear whether the presence of such a dead layer has any impact on the magnetic anisotropy. More film microstructure studies are undergoing to determine the origin of the "dead layer". 


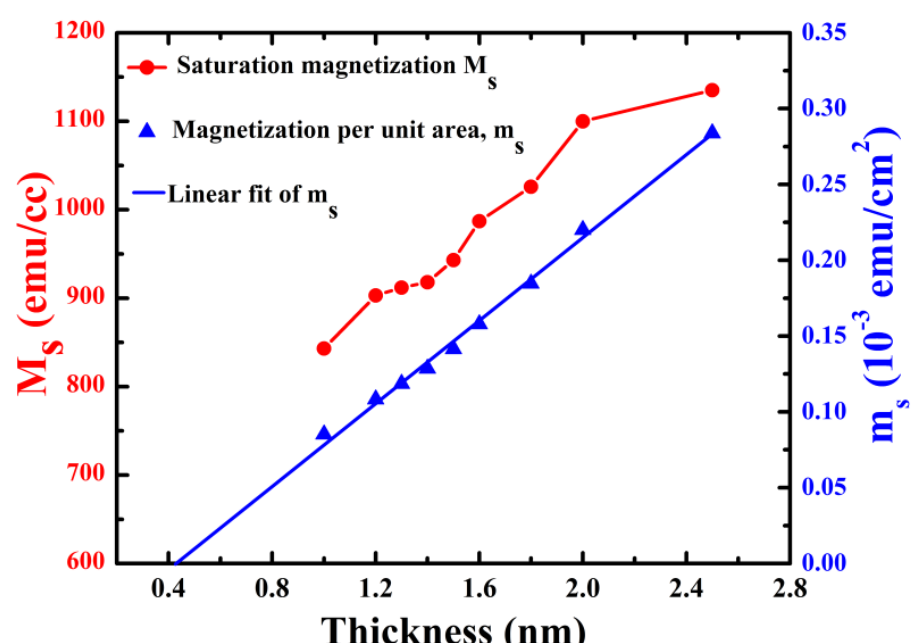

Figure 5.3 Saturation magnetization $M_{s}(\mathrm{emu} / \mathrm{cc})$ (red) and magnetization per unit area $\mathrm{m}_{\mathrm{s}}\left(\mathrm{emu} / \mathrm{cm}^{2}\right)$ (blue) vs the CoFeGe film thickness at $300 \mathrm{~K}$.

To separate the bulk and interfacial contribution of the anisotropy, the overall magnetic anisotropy energy density $K$, obtained from magnetization measurements, can be expressed by the equation $K=K_{b}-2 \pi M_{s}^{2}+K_{i} / t_{\mathrm{CoFeGe}}$ [see solid line in Fig. 5.4], where $K_{b}$ and $K_{i}$ are the bulk and interfacial anisotropy energy densities, respectively, and $2 \pi M_{s}^{2}$ is the demagnetization energy density. From the plot, it is clearly the anisotropy changes from out-of-plane to in-plane direction with the increase in CoFeGe film thickness. This transition occurs at thickness around $1.4 \mathrm{~nm}$, indicating that the interface plays an important role for PMA. From the intercept to the vertical axis, $K_{i}$ is estimated to be $0.9 \mathrm{erg} / \mathrm{cm}^{2}$, which is comparable to that of $\mathrm{CoFeB} / \mathrm{MgO}$ [77]. $K_{b}$ is obtained to be $-3 \times 10^{5} \mathrm{erg} / \mathrm{cm}^{3}$ from the slope of the linear extrapolation, indicating the bulk anisotropy contributes to the in-plane anisotropy. Thus, the perpendicular anisotropy in this structure is entirely due to the interfacial perpendicular anisotropy between $\mathrm{MgO}$ and CoFeGe. 


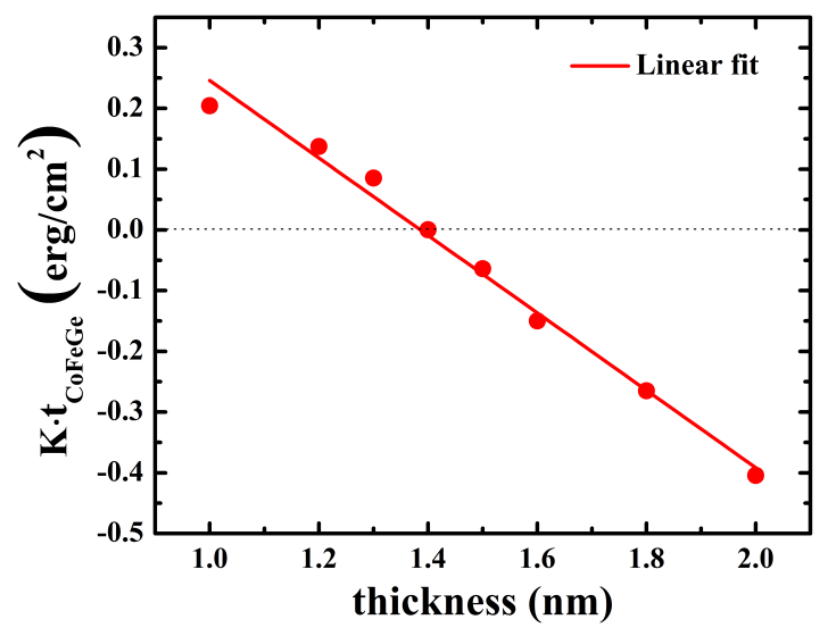

Figure 5.4 The dependence of total perpendicular anisotropy K on film thickness, plotted as the product of $K$ and thickness vs. thickness.

The interfacial perpendicular anisotropy between oxide and ferromagnetic metal has been predicted by first-principles calculation and attributed to hybridization of Co or Fe $3 d$ and $\mathrm{O} 2 p$ orbitals [82] at the interface of $\mathrm{MgO}$ and CoFeGe. The hybridization of $3 d z^{2}$ and $2 p z$ orbitals reduces the binding energy of $\mathrm{Co}-\mathrm{O}$ or $\mathrm{Fe}-\mathrm{O}$ perpendicular to the interface between $\mathrm{CoFeGe}$ and $\mathrm{MgO}$, which becomes lower than that of orbitals lying in the plane, resulting in strong PMA at the interface $[82,83]$. The PMA originates at the interface between the CoFeGe and MgO and is very sensitive to the thickness of each layer. Based on above, the effect of the thickness of CoFeGe $\left(t_{\mathrm{CoFeGe}}\right)$ is studied using samples with fixed thickness of $\mathrm{MgO}$ layer $\left(t_{\mathrm{MgO}}\right)(5.0 \mathrm{~nm})$. The effects of $t_{\mathrm{MgO}}$ on the magnetic properties of the annealed $\mathrm{MgO}(001) / \mathrm{CoFeGe} / \mathrm{MgO}$ multilayer films are, therefore, examined here. With the thickness of CoFeGe layer kept at an optimized 1.0nm, In-plane and out-of-plane hysteresis loops were taken from $\mathrm{MgO}(001) / \mathrm{CoFeGe}(1.0$ $\mathrm{nm}) / \mathrm{MgO}\left(t_{\mathrm{MgO}}\right) / \mathrm{Ta}(5 \mathrm{~nm})$. The Ta capping here was to protect films from oxidation. The samples with $t_{\mathrm{MgO}}=0 \mathrm{~nm}$ exhibit in-plane anisotropy with a high anisotropic field of about $1 \mathrm{~T}$ (not shown). Notably, this sample has a considerable residual in-plane magnetic component, 
which has not been observed in previous studies. PMA emerges with the insertion of 0.8-nmthick MgO layer, revealing that the interface in CoFeGe/MgO structure is essential for PMA. PMA increases with increasing $t_{\mathrm{MgO}}$ and is greatest in the sample with $t_{\mathrm{MgO}}=2.0 \mathrm{~nm}$, as shown in Fig. 5.5. The PMA energy density $K$ at $t_{\mathrm{MgO}}=2.0 \mathrm{~nm}$ is calculated to be $2 \times 10^{6} \mathrm{erg} / \mathrm{cm}^{3}$, comparable to that of other PMA systems such as $\mathrm{CoFeB} / \mathrm{MgO}[84]$ and $\mathrm{Co} / \mathrm{Pd}$ perpendicular multilayers [85], and can satisfy the high thermal stability for devices with lower than $50 \mathrm{~nm}$ dimension.

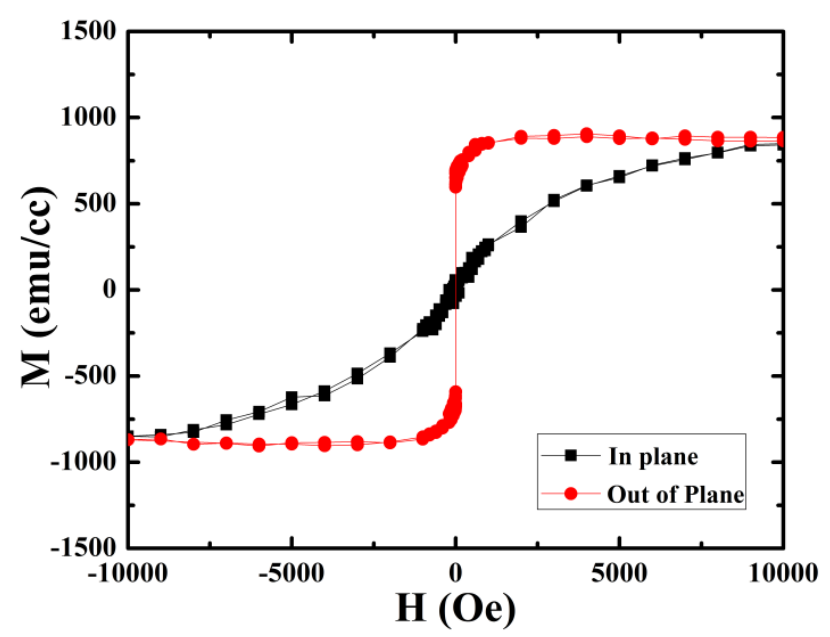

Figure 5.5 In-plane (black) and out-of-plane (red) hysteresis loops for the annealed sample of $\mathrm{MgO}(001) / \mathrm{CoFeGe}(1.0 \mathrm{~nm}) / \mathrm{MgO}(2.0 \mathrm{~nm}) / \mathrm{Ta}(5 \mathrm{~nm})$.

In order to determine the Gilbert damping constant in the films ferromagnetic resonance (FMR) spectra were recorded using a broadband coplanar waveguide field-swept FMR setup, with an upper frequency limit of about $50 \mathrm{GHz}$. Those FMR measurements were one by T. Mewes' team in University of Alabama. The resonance field dependence of the resonance frequency is well described by Kittel's formula. The effective magnetization determined from this is in good agreement with the saturation magnetization determined by VSM. The gyromagnetic ratio $\gamma$ shows no significant variation with the reported value [78]. 


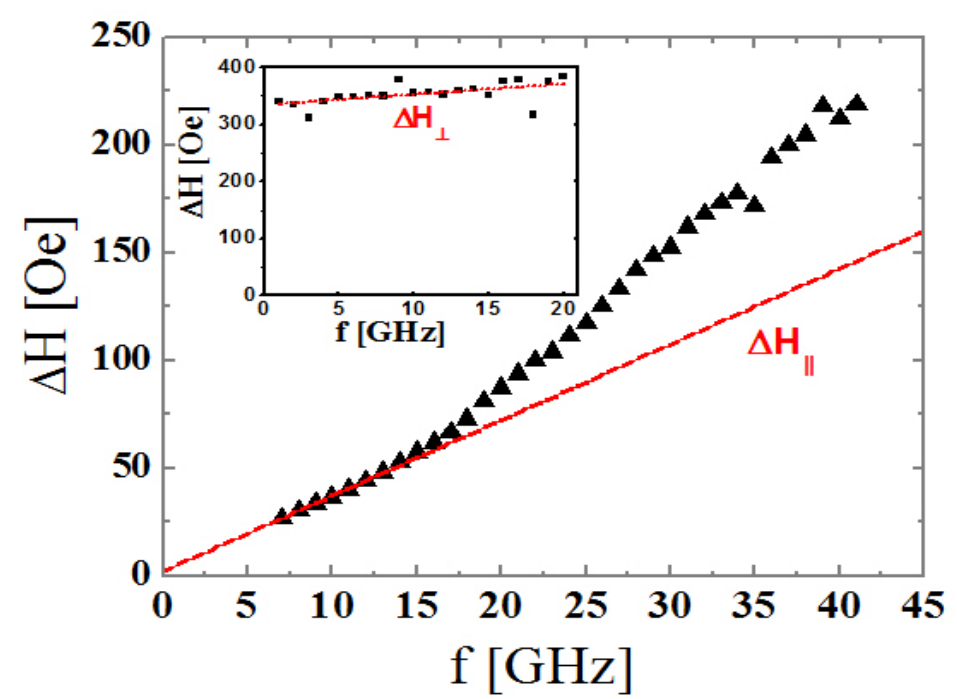

Figure. 5.6 Frequency dependence of the linewidth for a $2.5 \mathrm{~nm}$ thick $\mathrm{Co}_{20} \mathrm{Fe}_{50} \mathrm{Ge}_{30}$ alloy film (annealed for $2 \mathrm{~h}$ at $250^{\circ} \mathrm{C}$ ) with the field applied in the film plane and along the film normal (inset).Courtesy of $\mathrm{T}$. Mewes' work from University of Alabama.

For field-swept FMR experiments, the resonance frequencies dependence of the linewidth can be expressed as,

$$
\Delta \mathrm{H}=\Delta \mathrm{H}_{0}+\frac{2 \alpha f}{\sqrt{3} \gamma^{\prime}}
$$

where $\gamma^{\prime}=\gamma / 2 \pi$ and $\alpha$ is the dimensionless Gilbert damping parameter, $\Delta H_{0}$ is referred as an inhomogenous residual linewidth present. A typical linewidth of the thin film stack $\mathrm{MgO} / \mathrm{CoFeGe}(2.5 \mathrm{~nm}) / \mathrm{MgO}(5 \mathrm{~nm})$ annealed at $T_{a}=250{ }^{\circ} \mathrm{C}$ is shown in Figure 5.6, with applied field parallel and perpendicular to the film plane. For the in-plane FMR measurement, $\Delta H$ becomes increasingly nonlinear with $f$ at higher frequencies. Such a nonlinear behavior is related to the inhomogeneous contribution to the linewidth caused for example by the anisotropy distribution of the different grains in the sample or the presence of two-magnon scattering in ultrathin films [86]. Consequently, in the perpendicular configuration, two-magnon scattering is suppressed. We present $\Delta \mathrm{H}$ versus $f$ data measured with the applied field perpendicular to the 
film (inset in Fig. 5.6). Clear linear dependence on frequency is observed. The Gilberting damping constant $\alpha$ was evaluated as $\sim 0.005$ directly from the linear fit in perpendicular configuration.

\subsection{Summary}

In conclusion, we have fabricated (001)-oriented $\mathrm{Co}_{20} \mathrm{Fe}_{50} \mathrm{Ge}_{30}$ epitaxial films with the $B 2$ crystal structure. We have demonstrated the perpendicular easy axis in Fe-rich CoFeGe thin film originating from the interfacial perpendicular magnetic anisotropy between CoFeGe and MgO. Its perpendicular magnetic anisotropy is found to depend strongly on the thickness of the CoFeGe and MgO layers. A perpendicular magnetic anisotropy energy density of $2 \times 10^{6}$ $\mathrm{erg} / \mathrm{cm}^{3}$ was achieved in the CoFeGe thin films, which suggests the feasibility of the application of CoFeGe to the perpendicular ferromagnetic electrodes of MTJs with high thermal stability and low magnetic damping at reduced dimension. There may be more factors responsible for large PMA in the present structures containing a thinner CoFeGe, which will be investigated in the future. However, it is important to mention that the present results are of significant practical importance toward the realization of high density spin transfer torque magneto-resistive random access memory (STT MRAM), because these structures can be combined to form an MgO-based perpendicular MTJ. 


\section{Crystalline Alloys}

\subsection{Introduction}

Remarkable interest has increased in the search for crystalline thin films with perpendicular magnetization, which are good candidates for pMTJs because they generally have large perpendicular magnetic anisotropy of the order of $10^{7} \mathrm{erg} / \mathrm{cc}$. This high $\mathrm{K}_{\mathrm{u}}$ value is very important for obtaining good thermal stability for nanometer sized elements. $\mathrm{Mn}_{x} \mathrm{Ga}_{1-\mathrm{x}}(x=70-75)$ with $\mathrm{DO}_{22}$ crystal structure has the potential to possess high PMA according to previous theoretical and experimental results. In this structure, an atomic layer of $\mathrm{Mn}$ and a layer containing both $\mathrm{Mn}$ and $\mathrm{Ga}$ atoms are arranged periodically along the $c$-axis [Figure 6.1]. Classic neutron scattering experiments showed that this material had a ferromagnetic structure and the magnetic moments of Mn atoms at sites I and II [Fig. 6.1] were $2.8 \pm 0.3 \mu B$ and $1.6 \pm$ $0.2 \mu B$, respectively. Consequently, a low $M_{S}$ can be observed at room temperature although the Curie temperature is higher than $770 \mathrm{~K}$ [87]. The theoretically calculated spin polarization value as high as $88 \%$ at the Fermi level originates from the strong difference in conductivity for minority and majority electrons [88].

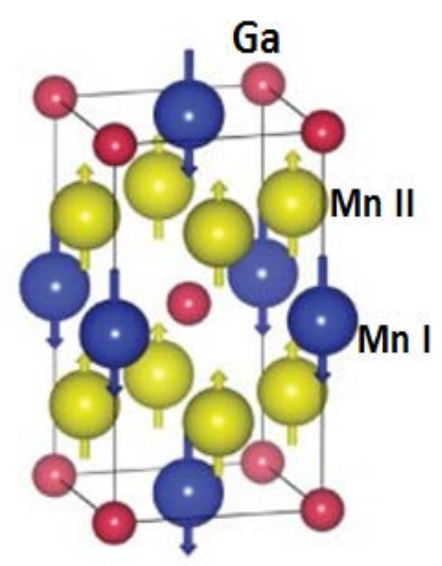

Figure 6.1. Lattice units of $\mathrm{DO}_{22}-\mathrm{Mn}_{2.5} \mathrm{Ga}$, arrows stand for magnetic moment directions. 


\subsection{Experiments}

In this work, 50 -nm-thick $\mathrm{Mn}_{2.5} \mathrm{Ga}$ films were prepared by co-deposition of elemental targets on $40 \mathrm{~nm}$-Cr-buffered MgO (001) substrates under base pressure less than $10^{-6}$ Torr. The deposition temperature $\left(T_{S}\right)$ of $\mathrm{MnGa}$ films varied from 250 to $400^{\circ} \mathrm{C}$. After deposition, each film was capped with a $5 \mathrm{~nm}-\mathrm{MgO}$ layer to prevent films from oxidation.

\subsection{Results and Discussion}

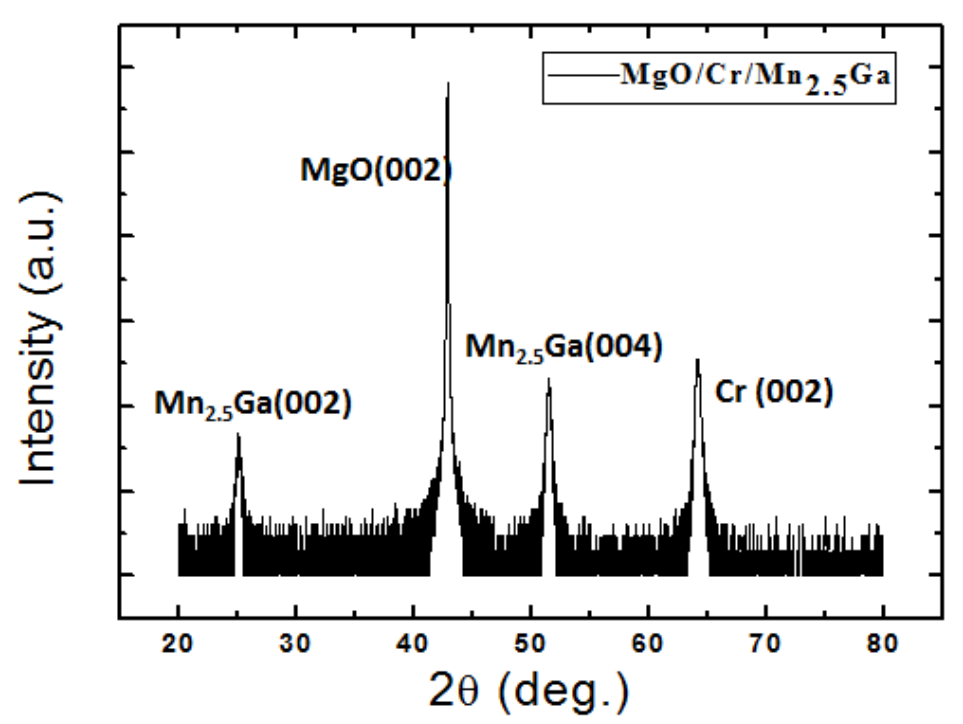

Figure $6.2 \mathrm{XRD} \theta-2 \theta$ pattern of $50-n \mathrm{mMn} n_{2.5}$ Ga film prepared at the substrate temperature of $400^{\circ} \mathrm{C}$.

Figure 6.2 shows a typical XRD $\theta-2 \theta$ pattern of the $\mathrm{Mn}_{2.5}$ Ga film prepared at the substrate temperature of $400^{\circ} \mathrm{C}$. In addition to peaks from $\mathrm{MgO}$ and $\mathrm{Cr}$, only $\mathrm{Mn}_{2.5} \mathrm{Ga}$ (002) and (004) peaks can be observed, indicating that $\mathrm{Mn}_{2.5} \mathrm{Ga}$ films were grown with the tetragonal $c$ axis along the normal direction. Since XRD patterns of the $\mathrm{DO}_{22}$ and $L 1_{0}$ crystal structure are quite similar [89,90], it is impossible to differentiate between them using only $\theta$ $2 \theta$ scans for our films. Hence, pole figure measurements ( $\Phi$-scan) were employed to confirm the crystal structure of $\mathrm{Mn}_{2.5} \mathrm{Ga}$ films. The presence of fourfold (011) peaks in all $\mathrm{Mn}_{2.5} \mathrm{Ga}$ films clearly indicates that the films have crystallized into the $\mathrm{DO}_{22}$ structure because the 
(011) peak is a forbidden diffraction peak in the $L 1_{0}$ crystal structure. To determine the epitaxial relationships between the $\mathrm{Mn}_{2.5} \mathrm{Ga}$ film and $\mathrm{Cr} / \mathrm{MgO}$ substrate, we measured the $\Phi$ scans of the $\mathrm{MgO}$ (022), $\mathrm{Cr}$ (022), and $\mathrm{Mn}_{2.5} \mathrm{Ga}$ (024) peaks. The result for the film prepared at $400{ }^{\circ} \mathrm{C}$ is displayed in Figure 6.3. The epitaxial relationship can be determined as $\mathrm{Mn}_{2.5} \mathrm{Ga}(001)[100] \| \mathrm{Cr}(001)[110]|| \mathrm{MgO}(001)[100]$, which also existed in films deposited at other temperatures. Lattice constants of $c$ and $a$ for epitaxial $\mathrm{Mn}_{2.5} \mathrm{Ga}$ films were calculated. The value of $a=3.909 \AA$ is approximately equal to the bulk value while the value of $c=7.09 \AA$ was found to be almost constant in the temperature region from 250 to $400{ }^{\circ} \mathrm{C}$.

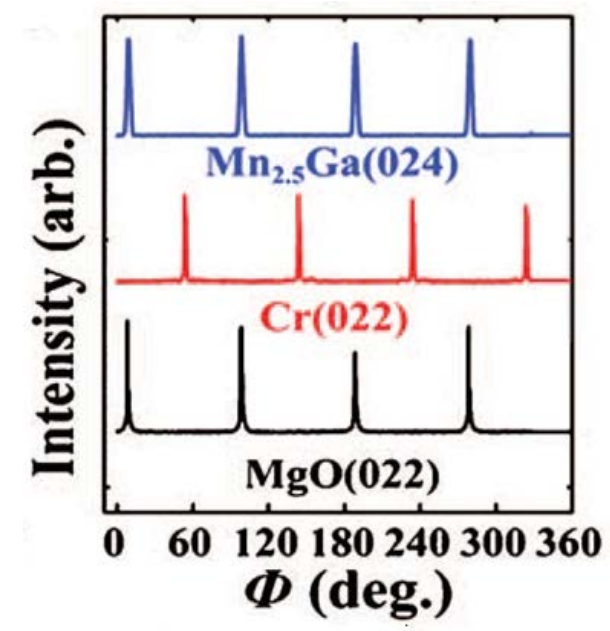

Figure $6.3 \Phi$-scan of the $\mathrm{Mn}_{2.5} \mathrm{Ga} / \mathrm{Cr} / \mathrm{MgO}$ structure in which $\mathrm{Mn}_{2.5} \mathrm{Ga}$ layer was prepared at $400^{\circ} \mathrm{C}$.

The in-plane and out-of-plane $M-H$ curves of the film prepared at $400{ }^{\circ} \mathrm{C}$ are given in Fig. 6.4. A well-squared loop is observed for the out-of-plane $M-H$ curve. However, the inplane $M-H$ curve exhibits almost zero remnant magnetization and cannot be saturated even under a magnetic field of $15 \mathrm{kOe}$. This result indicates that the easy axis of the magnetization is perpendicular to film plane. Also, this feature holds for the films prepared at other temperatures. 


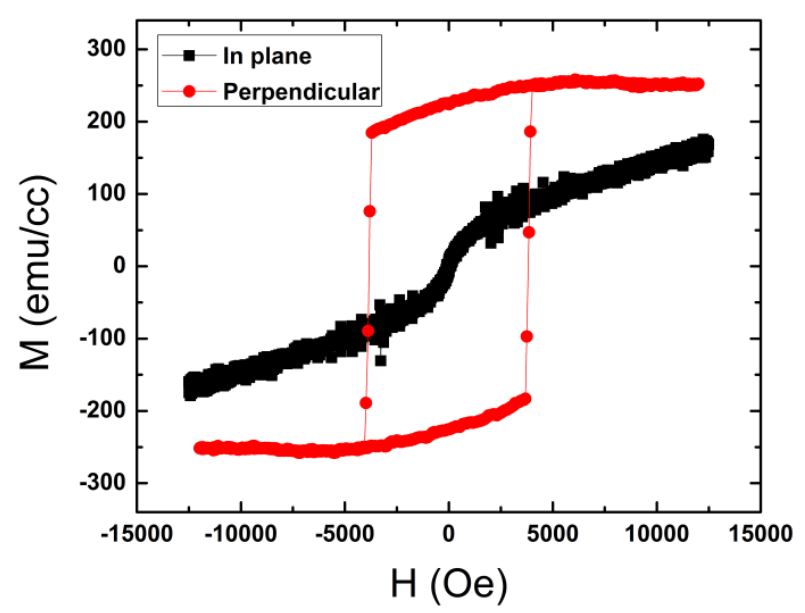

Figure 6.4. In-plane and out-of-plane M-H curves of the epitaxial Mn2.5Ga film prepared at $400{ }^{\circ} \mathrm{C}$.

The dependence of saturation moments $\left(M_{s}\right)$ on the deposition temperature $\left(T_{s}\right)$ for epitaxial $\mathrm{Mn}_{2.5} \mathrm{Ga}$ films is shown in Figure 6.5, where the value of $M_{S}$ was obtained from the out-of-plane $M-H$ curve. From 250 to $400{ }^{\circ} \mathrm{C}$, an increase in $M_{S}$ is observed. Further increase in $T_{S}$ has a little effect on $M_{S}$, and $M_{S}$ stays at a nearly invariant value of 260 emu $/ \mathrm{cm}^{3}$. As mentioned above, obtaining a PMA material with low $M_{s}$ is crucial for applications in spintronic devices. In this respect, our films are superior to the $L 1_{0}$-ordered PMA films, like FePt and CoPt [91], and can be comparable with the well-known ferrimagnetic PMA films, e.g., TbFeCo [92]. 


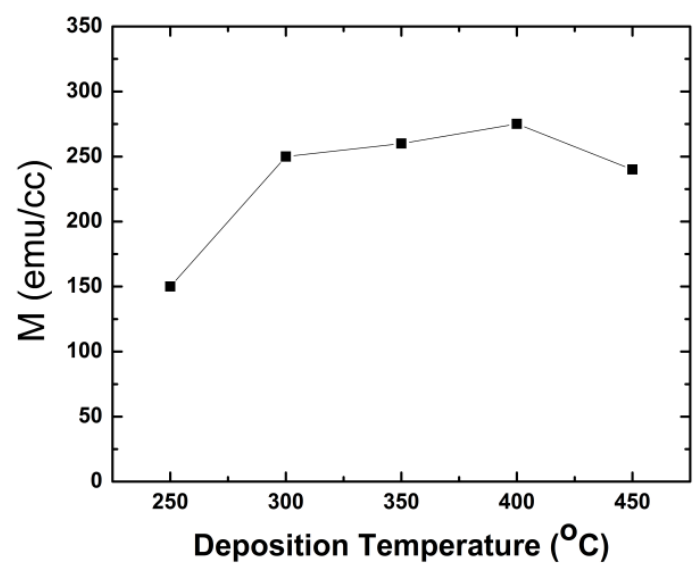

Figure 6.5. saturation magnetizations $\left(M_{s}\right)$ of MnGa films deposited at different temperatures.

To evaluate the PMA properties quantitatively, the perpendicular magnetic anisotropy energy $\left(K_{u}\right)$ was estimated using the relations $K_{u}=M_{s} H_{k} / 2$. Here, the anisotropy field $\left(H_{k}\right)$ was defined as the extrapolated intersection of the in-plane $M-H$ curve with the saturation magnetization value of the out-of-plane $M-H$ curve. $K_{u}$ increased monotonically and reach maximum values at $400{ }^{\circ} \mathrm{C}$ and then decrease with further increase in Ts. The highest $K_{u}$ value $\left(0.5 \times 10^{7} \mathrm{erg} / \mathrm{cm}^{3}\right)$ is comparable to that of other high PMA films, e.g., PtFe and CoPt [91,93]. Considering that the growth temperature of PMA films should be as low as possible for practical applications in spintronic devices, the epitaxial $\mathrm{Mn}_{2.5} \mathrm{Ga}$ film is very promising because high PMA can be obtained at relative low growth temperature.

In summary, we investigated the effects of deposition temperature on structural and magnetic properties of epitaxial $\mathrm{Mn}_{2.5} \mathrm{Ga}$ films. At optimum growth condition, the film exhibits giant PMA $\left(K u=0.5 \times 10^{7} \mathrm{erg} / \mathrm{cm}^{3}\right)$ and low saturation magnetization $\left(M_{s}=260 \mathrm{emu} / \mathrm{cm}^{3}\right)$. Further experiments are being carried out to testify its high spin polarization. 


\section{Tunable Perpendicular Magnetic Anisotropy in amorphous thin films}

\subsection{Introduction}

For devices, it is preferable to use a single film layer with perpendicular magnetic anisotropy instead of multilayer in order to avoid complicated fabrication process and decrease the total thickness of the devices. One well-known candidate is amorphous rare-earth transitionmetal (RE-TM) thin films with strong perpendicular magnetic anisotropy [94-97].

Amorphous GdFeCo films have been known to have a low saturation magnetization, preventing magnetization curling at the film edge. Furthermore, they possess Curie temperatures well above room temperature for a wide range of compositions [98]. Gd is a unique member of the lanthanide series in that its ground-state electronic configuration is $4 f^{7}(5 d 6 s)^{3}$ with the highest possible number of majority spin electrons and no minority spin electron in its $4 f$ state according to Hund's rule. In addition, because the $4 f$ states of $\mathrm{Gd}$ are half filled, their orbital moment and spin-orbit coupling are zero. This $\mathrm{L}=0$ state of $\mathrm{Gd}$ provides a favorable condition for low Gilbert damping, which is preferable in spin-torque-transfer devices. Amorphous GdFeCo alloys are ferrimagnets in which the $\mathrm{Fe}(\mathrm{Co})$ sublattices are antiferomagnetically coupled to the Gd sublattice in a collinear alignment, while the exchange coupling in the $\mathrm{Fe}(\mathrm{Co})$ sublattice is ferromagnetic [99]. These ferrimagnetic GdFeCo alloys tend to exhibit magnetic compensation behavior characterized by a vanishing magnetization below the Curie temperature [100]. Also, depending on the composition, amorphous GdFeCo films generally possess a uniaxial anisotropy with an anisotropy axis either perpendicular or parallel to the film plane.

In this study, we have obtained amorphous GdFeCo films for a wide range of Gd content via the combinatorial growth technique. We have investigated the compositional and temperature dependence of magnetization compensation in these amorphous ferrimagnetic $\mathrm{Gd}_{x} \mathrm{Fe}_{93-\mathrm{x}} \mathrm{Co}_{7}$ 
films and demonstrated the tunability of perpendicular magnetic anisotropy. Possible mechanisms for the observed perpendicular magnetic anisotropy are discussed.

\subsection{Experimental procedure}

The $\mathrm{Gd}_{\mathrm{x}} \mathrm{Fe}_{93-\mathrm{x}} \mathrm{Co}_{7}(\mathrm{GdFeCo})$ films were prepared at ambient temperature on thermally oxidized Si substrates using $r f$ magnetron sputtering. The base pressure of the sputtering chamber was $\sim 7 \times 10^{-7}$ Torr. GdFeCo alloy films were deposited by means of co-sputtering with the elemental targets under a processing Ar gas pressure around $5 \times 10^{-3}$ Torr. The capping MgO layer was formed directly from a sintered MgO target to protect GdFeCo layer from oxidation. All the samples deposited at room temperature had a typical structure consisting of $\mathrm{Si}(100) / \mathrm{SiO}_{2} / \mathrm{Gd}_{\mathrm{x}} \mathrm{Fe}_{93-\mathrm{x}} \mathrm{Co}_{7}(15 \leq x \leq 59$ at. \%)/MgO(6 nm) with a fixed thickness of GdFeCo layer $\sim 50 \mathrm{~nm}$.

\subsection{Results and discussions}

The amorphous structures of the as-deposited samples were confirmed by TEM and XRD observations. Fig. 7.1(a) shows a typical cross-sectional TEM image obtained from as-deposited $\mathrm{Gd}_{22} \mathrm{Fe}_{71} \mathrm{Co}_{7}$ film on the $\mathrm{Si} / \mathrm{SiO}_{2}$ substrate. The microstructure was dense with no visible cracks or holes, and all layers in the structure were well adhered to each other. The uniform thickness of $\mathrm{Gd}_{22} \mathrm{Fe}_{71} \mathrm{Co}_{7}$ film was measured to be $52 \mathrm{~nm}$ in Fig. 7.1(a). The high resolution TEM image in Fig. 7.1(b) revealed the featureless nanoscale structure that indicated the lack of long-range order. The broad ring pattern in the fast Fourier-transform (FFT) image in the inset of Fig. 7.1(b) indicated the lack of crystallinity. In addition, XRD scans showed that there were no diffraction peaks other than those from the substrate for GdFeCo films with different compositions (not shown here). The XRD results were consistent with the TEM, indicating that all the as-deposited samples were amorphous in nature, without the formation of a long-range structural order. 
Atomic force microscopy (AFM) measurements showed that the surfaces were free of pinholes and were flat with roughness less than $1 \mathrm{~nm}$.
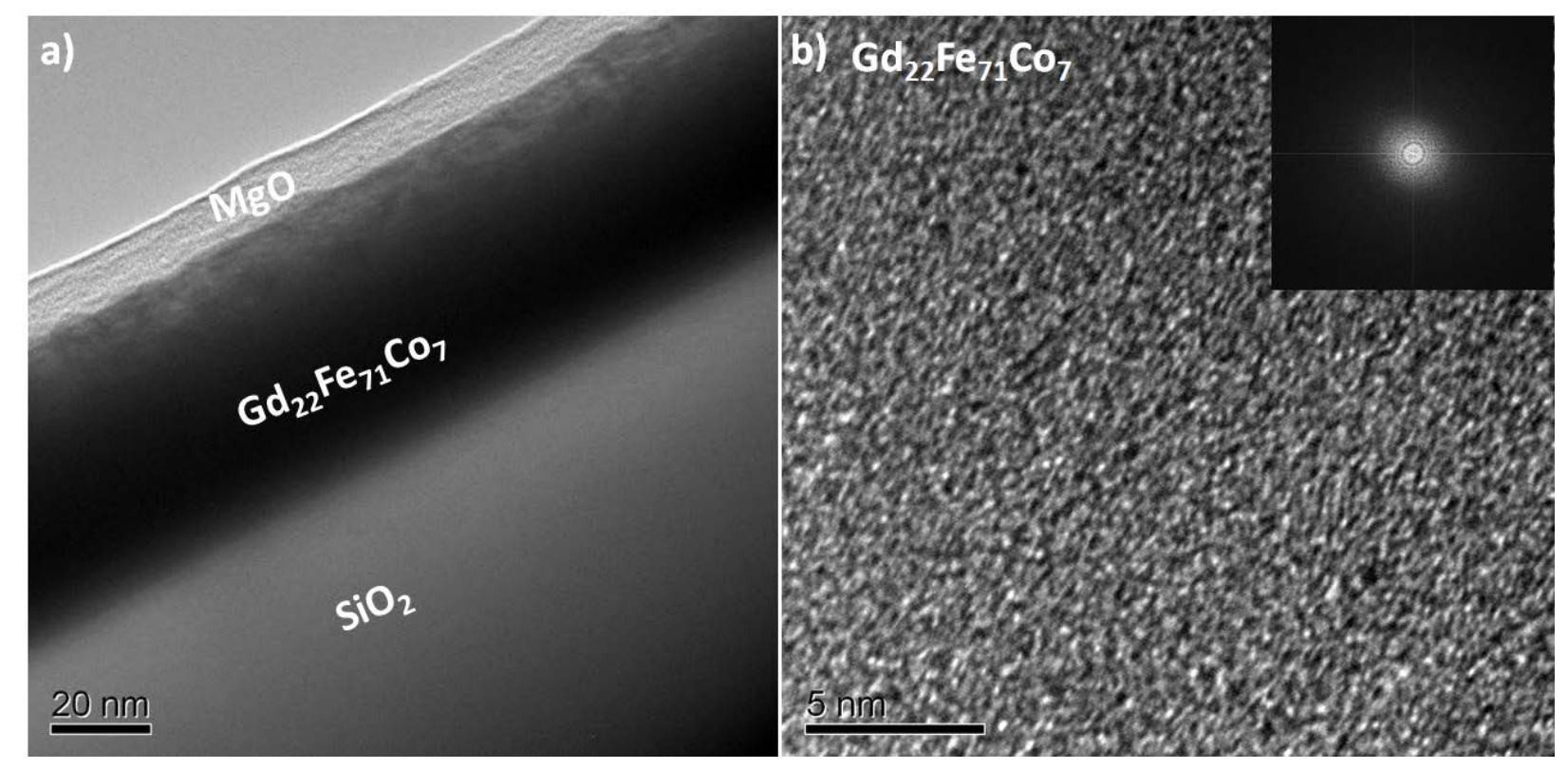

Figure 7.1 (a) Cross-sectional TEM image of as-deposited $\mathrm{Gd}_{22} \mathrm{Fe}_{71} \mathrm{Co}_{7}$ film on $\mathrm{SiO}_{2} / \mathrm{Si}$ substrate. $6 \mathrm{~nm}$ $\mathrm{MgO}$ layer was used to cap the film; (b) high-resolution TEM image of the $\mathrm{Gd}_{22} \mathrm{Fe}_{71} \mathrm{Co}_{7}$ film. The inset is a FFT pattern of the image.

\subsubsection{Low Gd-content films}

The magnetization of GdFeCo films were characterized in the in-plane and out-of-plane directions using the VSM option in Quantum Design VersaLab. Fig. 7.2 shows the temperature dependence of saturation magnetization of as-deposited GdFeCo films for several compositions. Saturation magnetization $\left(M_{s}\right)$ was extracted from the hysteresis loops measured as a function of temperature between $100 \mathrm{~K}$ and $400 \mathrm{~K}$. Compensation temperature ( $\left.T_{\text {comp }}\right)$ was defined as the temperature at which $M_{s}(\mathrm{~T})$ reached its minimum. The saturation moments at the compensation temperatures were below $100 \mathrm{emu} / \mathrm{cc}$. The observed small saturation moment was due to the ferrimagnetism of amorphous RE-TM alloys. The TM-TM ferromagnetic interaction aligns the magnetic moments among Fe and Co ions, which are coupled antiferromagnetically with the 
magnetic moments of $\mathrm{Gd}$. As a result, the net moment is the difference between the magnetic moments of $\mathrm{Gd}$ and $\mathrm{Co}(\mathrm{Fe})$. At the compensation temperature, the moments of the two magnetic sublattices were nearly equal, giving rise to a low saturation magnetization.

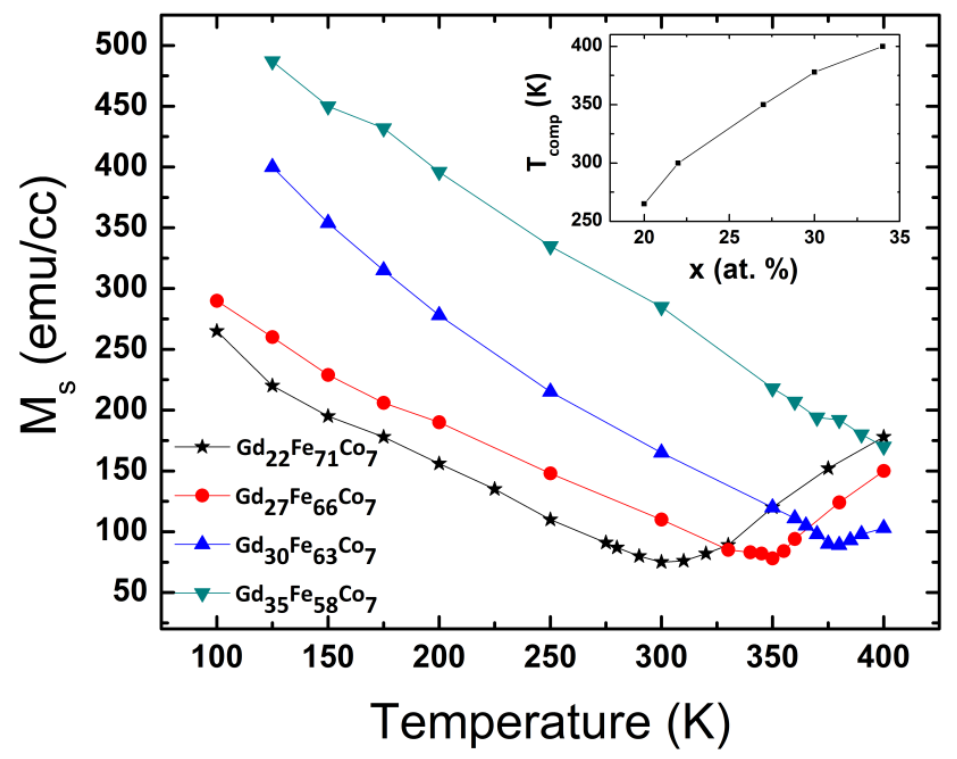

Figure7.2 Temperature dependence of the saturation magnetization of as-deposited GdFeCo films with various $G d$ concentrations ( $x=22,27,30$ and 35). The inset in (b) shows the dependence of the magnetization compensation temperature $T_{\text {comp }}$ on the Gd concentration.

Due to the different temperature dependence of the sublattice magnetizations, compensation temperature can be varied, depending on the compositions. The variation of $M_{s}$ with temperature (Fig. 7.2) resembled the expected compensation behavior when approaching the compensation point. The GdFeCo films with $\mathrm{x}=22,27$ and 30 at. \% exhibited the compensation temperatures $T_{\text {comp }}$ at $300 \mathrm{~K}, 350 \mathrm{~K}$ and $378 \mathrm{~K}$, respectively. However, for several other compositions, there was no compensation point within the investigated temperature interval from 100 to $400 \mathrm{~K}$. For $\mathrm{Gd}_{35} \mathrm{Fe}_{58} \mathrm{Co}_{7}$ film, the compensation point was not obtained due to the limitation of the measurement temperature range. For the sample $\mathrm{Gd}_{15} \mathrm{Fe}_{78} \mathrm{Co}_{7}$, the dependence of saturation magnetization on $T$ (not shown) indicated that the magnetization of the $\mathrm{Fe}(\mathrm{Co})$ 
sublattices exceeded the magnetization of the Gd sublattice in the whole temperature range. This was due to the fact that ferromagnetic exchange of the $\mathrm{Fe}(\mathrm{Co})$ sublattices dominates the magnetic behavior at low Gd content. As the Gd content increases and $\mathrm{Fe}(\mathrm{Co})$ content decreases, there is a corresponding increase in the antiferromagnetic coupling relative to the ferromagnetic exchange, and magnetic compensation emerges. Further increase in the Gd concentration resulted in the increase in $T_{\text {comp }}$, as shown in the inset to Fig. 7.2.

According to magnetization measurements, the perpendicular magnetic anisotropy in GdFeCo films appeared near their compensation temperatures, whereas otherwise the magnetic easy axis was in-plane. Fig. 7.3(a) shows the typical normalized out-of-plane hysteresis loops of the as-deposited $\mathrm{Gd}_{27} \mathrm{Fe}_{66} \mathrm{Co}_{7}$ films at various temperatures. At $250 \mathrm{~K}$, far from $T_{\text {comp }} \sim 350 \mathrm{~K}$, magnetization was dominated by in-plane anisotropy. Near the compensation point, perpendicular magnetic anisotropy was dominant, and a square hysteresis loop was established in the out-of-plane direction with coercivity near 100 Oe.
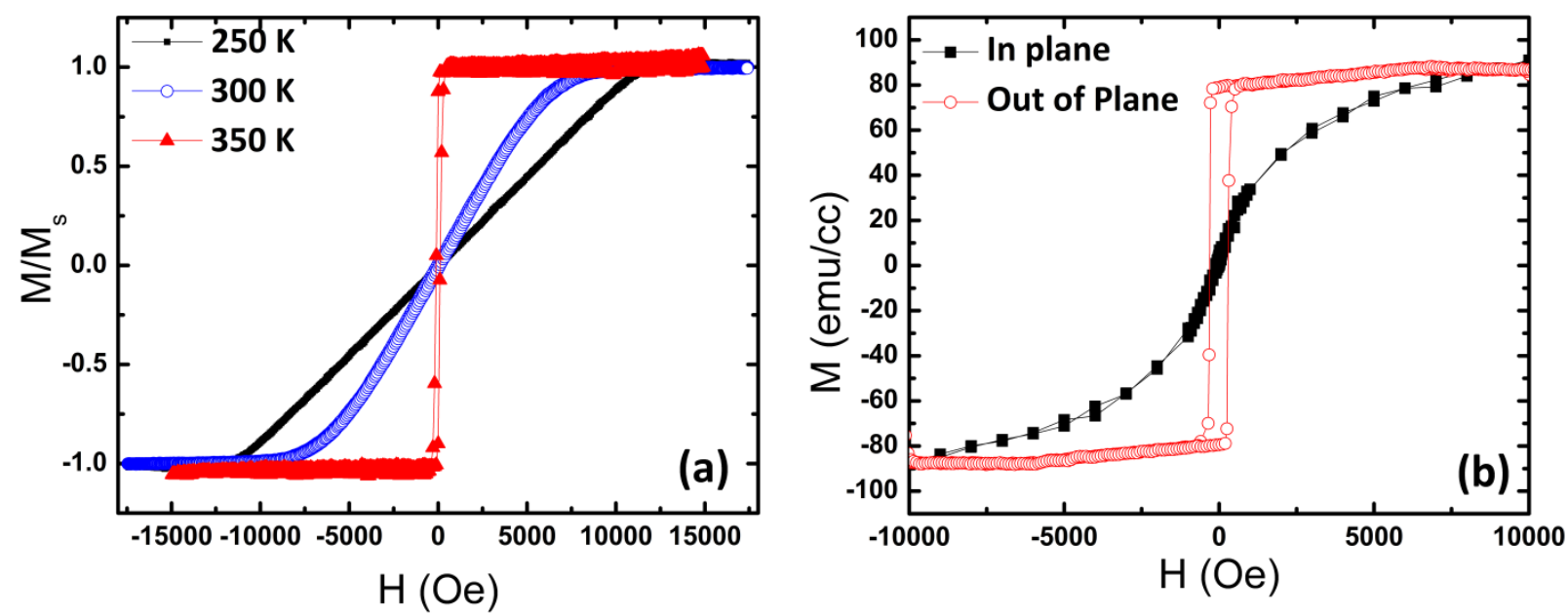

Figure 7.3 (a) Normalized out-of-plane hysteresis loops of as-deposited $\mathrm{Gd}_{27} \mathrm{Fe}_{66} \mathrm{Co}_{7}$ film measured at 250, 300, and $350 \mathrm{~K}$. (b) In-plane (black square) and out-of plane (red circle) hysteresis loops of $50 \mathrm{~nm}$ as-deposited $\mathrm{Gd}_{22} \mathrm{Fe}_{71} \mathrm{Co}_{7}$ film. 
From the magnetization variation curve (Fig. 7.2), the $\mathrm{Gd}_{22} \mathrm{Fe}_{71} \mathrm{Co}_{7}$ film had magnetic compensation temperature at room temperature, which has particular technological importance [101]. Fig. 7.3(b) shows the room-temperature in-plane and out-of-plane hysteresis (M-H) loops of the $50 \mathrm{~nm} \mathrm{Gd}{ }_{22} \mathrm{Fe}_{71} \mathrm{Co}_{7}$ film, indicating that this composition has PMA with out-of-plane coercivity of about 360 Oe. The total perpendicular anisotropy energy density $\left(K_{u}\right)$, which determines the thermal stability, was $3.8 \times 10^{5} \mathrm{erg} / \mathrm{cm}^{3}$, as calculated by evaluating the area enclosed between the in-plane and perpendicular M-H curves [102]. The out-of-plane loop showed sharp, square switching characteristics with a squareness of one (1). For $\mathrm{Gd}_{22} \mathrm{Fe}_{71} \mathrm{Co}_{7}$ film, a relatively small concentration of the high-moment RE magnetically compensated the lower moment TM at room temperature. Since the tunneling current in spintronics devices is dominated by that from the highly polarized TM atoms, this material would be very useful for device applications because it would eliminate magnetic dipole fields that can give rise to significant magnetic coupling within and between devices.

The above results underlined the fact that in GdFeCo system, the magnetization anisotropy may be easily tuned by adjusting the composition and/or the temperature. In the lowGd region of $x$ between 20 and 34, the Gd sublattice dominated the overall magnetization of the system below the compensation temperature. However, Above $T_{\text {comp }}$ the magnetization of the $\mathrm{Fe}(\mathrm{Co})$ sublattice prevailed and the total magnetization of the system continuously increased on further warming. Since the magnetic anisotropy can vary around this temperature, $T_{\text {comp }}$ is an important parameter that ensures the stability of the stored information.

\subsubsection{High Gd-content films}

At room temperature, amorphous GdFeCo films with Gd concentrations varying between $52 \%$ and $59 \%$ were found to exhibit low magnetization. Fig. 7.4 shows the temperature 
dependence of saturation magnetization of as-deposited GdFeCo films for Gd concentrations at 54\% and 57\%. The magnetization decreased precipitously with increasing temperature and finally decreased to near zero between 370 and $400 \mathrm{~K}$. Magnetic compensation temperature was not observed in this composition region, which was consistent with some recently reported computational results on GdFeCo films [103]. The film compositions with 54 and 57 at. \% Gd exhibited Curie temperatures at $375 \mathrm{~K}$ and $400 \mathrm{~K}$, respectively, much lower than $500 \mathrm{~K}$ of the composition with 22 at. \% Gd [104]. These can be attributed to a stronger role of antiferromagnetic coupling at high Gd content, which also tended to reduce the Curie temperature.

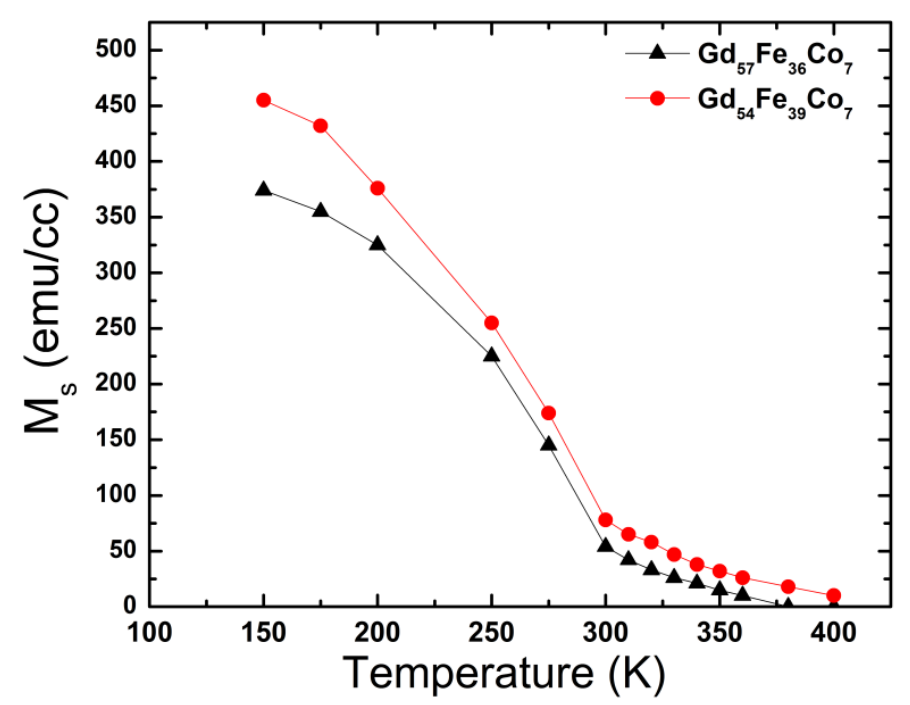

Figure 7.4. Temperature dependence of the saturation magnetization of GdFeCo films with various Gd concentrations ( $x=54$ and 57).

In view of the low magnetization, perpendicular magnetic anisotropy was investigated at room temperature for Gd content between 52\% to 59\%. Fig. 7.5(a) shows the compositional dependence of out-of-plane coercivity $\left(H_{c}\right)$ and saturation magnetization $\left(M_{s}\right)$ of $50 \mathrm{~nm}$ asdeposited perpendicularly magnetized GdFeCo films for $\mathrm{x}$ between 52 and 59. With the 
increasing content of $\mathrm{Gd}, M_{s}$ decreased from 100 to $55 \mathrm{emu} / \mathrm{cc}$ at $\mathrm{x}=57$, then increased to 84 emu/cc at $x=59$. However, the out-of-plane coercivity showed an opposite trend, which increased with increasing Gd concentration and was greatest in the sample with 57 at. \% Gd. Fig. 7.5(b) shows the typical hysteresis loops of as-deposited $\mathrm{Gd}_{57} \mathrm{Fe}_{36} \mathrm{Co}_{7}$ film with thickness of $50 \mathrm{~nm}$. A clear perpendicular anisotropy was realized with out-of-plane coercivity $H_{c}=6.6 \mathrm{kOe}$. The total perpendicular anisotropy energy density $K_{u}$, which determined the thermal stability, was $2.6 \times 10^{5}$ erg/cc. Generally, the RE ions exhibit large local magnetic anisotropy due to its spin-orbit coupling. $\mathrm{Gd}$ is in the $\mathrm{L}=0$ state, spin-orbit coupling is supposed to be small; however, the $5 \mathrm{~d}$ electrons have finite spin-orbit coupling which can be partially responsible for this anisotropy. Because of their large out-of-plane coercivity, ferrimagnetic GdFeCo films in the composition range $52 \leq \mathrm{x} \leq 59$ can be of technological importance in the area of the thermomagnetic recording devices.
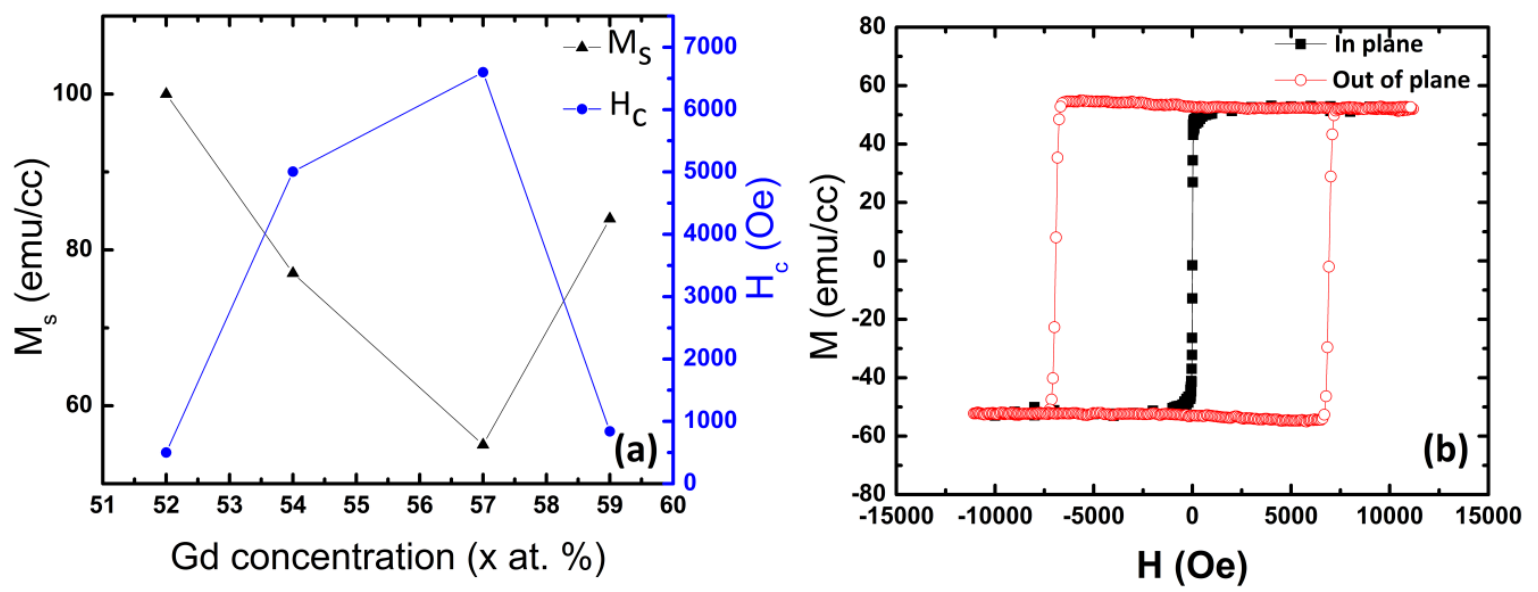

Figure 7.5 (a) Gd-content dependence of $M_{s}$ (black triangle) and $H_{c}$ (blue circle) for perpendicularly magnetized GdFeCo films between 52 and 59 at. \% Gd. (b) In-plane and out-of-plane hysteresis loops of $\mathrm{Gd}_{57} \mathrm{Fe}_{36} \mathrm{Co}_{7}$ film with thickness at $50 \mathrm{~nm}$. 


\subsubsection{Discussion}

Perpendicular magnetic anisotropy at room temperature was found in the compositions near 23 and 57 at. \% Gd. Since amorphous alloys lack structural long-range order, one might expect that atomic-scale structure plays an important role in determining the properties of these alloys. Studies have attempted to correlate the magnetic anisotropy of amorphous RE-TM films with various structural characteristics ranging from columnar textures [105] to microcrystallinity [106] to local magnetic anisotropy or/and atomic-scale anisotropy [107-109]. Cross-sectional TEM study (Fig. 7.1) failed to detect nanoscale columnar growth and microcrystallinity in the amorphous GdFeCo films. As discussed above, local magnetic anisotropy of rare earth atoms does not play a role in Gd-Fe-Co, unlike other rare earth elements such as $\mathrm{Tb}$ and Dy; and yet amorphous Gd-Fe-Co films show robust PMA behavior with a large $H_{c}$ comparable to that of amorphous Tb-Fe-Co films [110]. These findings suggest that the observed perpendicular magnetic anisotropy in amorphous GdFeCo films probably has a structural origin involving atomic-scale anisotropy. This atomic-scale order can be anisotropic in as-grown films, which influences the short-range exchange interaction, leading to anisotropic properties. The perpendicular anisotropy may arise from the change in nearest neighbor distance and the coordination numbers for RE and TM sublattices which can affect the short range order.

\subsection{Conclusion}

In summary, it was shown that the magnetic anisotropy of amorphous ferrimagnetic $\mathrm{Gd}_{\mathrm{x}} \mathrm{Fe}_{93-\mathrm{x}} \mathrm{Co}_{7}$ films can be controlled by varying the composition as well as temperature. The low-Gd region of $\mathrm{x}$ between 20 and 34 were found to exhibit compensation phenomena characterized by a low saturation magnetization and perpendicular magnetic anisotropy near the 
compensation temperature. Furthermore, low magnetizations and perpendicular magnetic anisotropy with large coercivity of $6.6 \mathrm{kOe}$ were observed at room temperature in previously unreported composition range of 52-59 at. \% Gd. No compensation temperature was measured in this range, which was consistent with recent model calculations. The observed perpendicular magnetic anisotropy in amorphous GdFeCo films probably has a structural origin consistent with certain aspects of the atomic-scale anisotropy. Our results have provided a way to fabricate GdFeCo films with tunable magnetic anisotropy by varying the composition or/and temperature, making these amorphous films attractive for future nanomagnetic devices. 


\section{Conclusions}

In this dissertation, we have investigated a wide range of materials with magnetic anisotropy varying from in-plane to fully perpendicular: from multilayer stacks and Co/Febased perpendicular crystalline alloys to amorphous GdFeCo. These materials hold a new generation of spintronics devices that can be integrated into conventional complementary metal- oxide semiconductor technology. These devices, when fully optimized, can be used in magnetic random access memory (MRAM), as well as spin transfer torque random access memory (STT- RAM) devices and also have potential applications in future spin-based quantum computer systems.

In chapter four, we have investigated the structural and magnetic properties of Cr-doped CoFeB films. Structure characterization revealed the crystallization sequence with a primary crystallization of BCC phase from an amorphous precursor followed by the precipitation of boride phases at a higher annealing temperature. In order to form BCC phase with Boride phase suppressed, specific film compositions were chosen with reduced B content accompanied by increasing Cr substitution. Film Compositions high in chromium showed a significant improvement in coercivity (500 Oe) and reduction in magnetization (300 emu/cc) after annealing, making it promising spintronic materials for achieving low switching current.

In chapter five and seven of the dissertation we systematically studied various perpendicular magnetic anisotropy (PMA) magnetic multilayered systems. The multilayered system we initially investigated is the epitaxial growth of CoFeGe/MgO structure. Structure characterization revealed (001)-oriented $B 2$ order of $\mathrm{Co}_{20} \mathrm{Fe}_{50} \mathrm{Ge}_{30}$ well lattice matched with the MgO barrier. Perpendicular magnetic anisotropy was achieved in the $\mathrm{MgO} / \mathrm{Co}_{20} \mathrm{Fe}_{50} \mathrm{Ge}_{30} / \mathrm{MgO}$ structure with an optimized magnetic anisotropy energy density of $2 \times 10^{6} \mathrm{erg} / \mathrm{cm}^{3}$. The 
magnetic anisotropy is found to depend strongly on the thickness of the $\mathrm{MgO}$ and $\mathrm{Co}_{20} \mathrm{Fe}_{50} \mathrm{Ge}_{30}$ layers, indicating that the perpendicular magnetic anisotropy of $\mathrm{Co}_{20} \mathrm{Fe}_{50} \mathrm{Ge}_{30}$ is contributed by the interfacial anisotropy between $\mathrm{Co}_{20} \mathrm{Fe}_{50} \mathrm{Ge}_{30}$ and $\mathrm{MgO}$. With reported low damping constant, $\mathrm{Co}_{20} \mathrm{Fe}_{50} \mathrm{Ge}_{30}$ films are promising spintronic materials for achieving low switching current.

In chapter six of the dissertation, we have investigated a different type of perpendicular magnetic anisotropy (PMA) materials, namely, $\mathrm{D}_{22}$ crystalline alloy. $\mathrm{D}_{22}$ alloy was initially investigated for advanced media applications; however, due to their high thermal stability coupled with perpendicular magnetic anisotropy, these alloys were subsequently investigated for perpendicular magnetic tunnel junctions. We report on epitaxial growth and magnetic properties of Mn2.5Ga thin films, which were deposited on $\mathrm{Cr} / \mathrm{MgO}$ single crystal substrates by magnetron sputtering. X-ray diffraction results revealed the epitaxial relationships as $\mathrm{Mn}_{2.5} \mathrm{Ga}(001)[100]\|\mathrm{Cr}(001)[110]\| \mathrm{MgO}(001)[100]$. The presence of (002) and (011) superlattice peaks indicates that the films were crystallized into $\mathrm{DO}_{22}$ ordered structures. A large $\mathrm{K}_{\mathrm{u}}=0.5 \times 10^{7} \mathrm{erg} / \mathrm{cc}$ and low magnetization of $260 \mathrm{emu} / \mathrm{cc}$ were obtained.

Finally, we have also investigated perpendicular magnetic anisotropy (PMA) of rare earth transition metal (RE-TM) alloys which can be used for free and pinned layers in pMTJs because of their tunable magnetic properties such as saturation magnetization and coercivity. The compositional and temperature dependence of magnetic compensation in amorphous ferrimagnetic $\mathrm{Gd}_{x} \mathrm{Fe}_{93-x} \mathrm{Co}_{7}$ alloy films were explored. Magnetic compensation is attributed to the competition between antiferromagnetic coupling of rare-earth (RE) with transition-metal (TM) ions and ferromagnetic interaction between the TM ions. The low-Gd region of $\mathrm{x}$ between 20 and 34 was found to exhibit compensation phenomena characterized by a 
low saturation magnetization and perpendicular magnetic anisotropy (PMA) near the compensation temperature. Compensation temperature was not observed in previously unreported high-Gd region of $\mathrm{x}=52-59$, in qualitative agreement with results from recent model calculations. However, low magnetization was achieved at room temperature, accompanied by a large PMA with coercivity reaching 6.6 kOe. The observed perpendicular magnetic anisotropy of amorphous GdFeCo films probably has a structural origin consistent with certain aspects of the atomic-scale anisotropy. Our findings have broadened the composition range of transition metal-rare earth alloys for designing PMA films, making it attractive for tunable magnetic anisotropy in nanoscale devices.

Another importance characterization parameters are chemical ordering type and the degree of certain chemical ordering based on the diffraction patterns, which is important for ordered alloys such as MnGa and CoFeGe and will be explored in future work. 


\section{References:}

[1] Igor Zutic, Jaroslav Fabian and S. Das Sarma. "Spintronics: Fundamentals and Applications.”Reviews of Modern Physics, 76 (2004).

[2] Chappert, A.Fert, and F.N. Van Dau, "The emergence of spin electronics in data storage." Nat Mater, 8 (2007) 813-823.

[3] B.Prince, M.S. Int, and T.X. Leander, "Treads in scaled and nanotechnology memories," Non-Volatile Memory Technology Symposium, 2005.

[4] Kinam Kim, "Future memory technology: challenges and opportunities," VLSI Technology, Systems and Applications, 2008.

[5] Jian-Gang Zhu,etc "Magnetoresistive random access memory: the path to competitiveness and scalability." Proceedings of the IEEE, 96 (2008) 1786-1798.

[6] R. C. O'Handley, "Modern Magnetic Materials: Principles and Applications," (John Wiley \& Sons, 2000).

[7] S. Parkin, X. Jiang, C. Kaiser, A. Panchula, K. Roche, and M. Samant, "Magnetically Engineered Spintronic Sensors and Memory" Proc. IEEE 91, 661 (2003).

[8] M. N. Baibich, J. M. Broto, A. Fert, F. N. Van Dau, F. Petroff, P. Etienne, G. Creuzet, A. Friederich, and J. Chazelas, "Giant Magnetoresistance of (001)Fe/(001)Cr Magnetic superlattices" Phys. Rev. Lett. 61 (21), 2472 (1988).

[9] G. Binasch, P. Grunberg, F. Saurenbach, and W. Zinn, "Enhanced magnetoresistance in layered magnetic structures with antiferromagnetic interlayer exchange" Phys. Rev. B 39 (7), 4828 (1989).

[10] S. S. P. Parkin, N. More, and K. P. Roche, "Oscillations in exchange coupling and magnetoresistance in metallic superlattice structures: $\mathrm{Co} / \mathrm{Ru}, \mathrm{Co} / \mathrm{Cr}$, and $\mathrm{Fe} / \mathrm{Cr}$ " Phys. Rev. Lett. 64 (19), 2304 (1990).

[11] M. Julliere, "Tunneling between ferromagnetic films" Phys. Lett. A 54, 225 (1975). [12] J. S. Moodera, L. R. Kinder, T. M. Wong, and R. Meservey, "Large Magnetoresistance at Room Temperature in Ferromagnetic Thin Film Tunnel Junctions" Phys. Rev. Lett. 74, 3273 (1995).

[13] S. S. P. Parkin, C. Kaiser, A. F. Panchula, P. Rice, M. G. Samant, S.-H. Yang, and B. Hughes, "Giant tunneling magnetoresistance at room temperature with MgO (100) tunnel barriers" Nature Materials 3, 862 (2004).

[14] S. Yuasa, T. Nagahama, A. Fukushima, Y. Suzuki, and K. Ando, "Giant roomtemperature magnetoresistance in single-crystal Fe/MgO/Fe magnetic tunnel junctions" Nature Materials 3, 868 (2004).

[15] S. Parkin, X. Jiang, C. Kaiser, A. Panchula, K. Roche, and M. Samant, "Magnetically Engineered Spintronic Sensors and Memory" Proc. IEEE 91, 661 (2003).

[16] S. Ikeda, J. Hayakawa, Y. Ashizawa, Y. M. Lee, K. Miura, H. Hasegawa, M. Tsunoda, F. Matsukura, and H. Ohno, "Tunnel magnetoresistance of 604\% at 300K at suppression of Ta diffusion in $\mathrm{CoFeB} / \mathrm{MgO} / \mathrm{CoFeB}$ pseudo-spin-valves annealed at high temperature" Appl. Phys. Lett. 93, 082508 (2008) 
[17] S. Maekawa and U. Gafvert, "Electron tunneling between ferromagnetic films" IEEE Trans. Magn. 18, 707 (1982).

[18] J.Nowak andJ. Rauluszkiewicz, "Spin dependent electron tunneling between ferromagnetic films" J. Magn. Magn. Mater. 109, 79 (1992).

[19] T. Miyazaki and N. Tezuka, "Giant magnetic tunneling effect in $\mathrm{Fe} / \mathrm{Al}_{2} \mathrm{O}_{3} / \mathrm{Fe}$ junction," J. Magn. Magn. Mater. 139, L231 (1995).

[20] J.S. Moodera, L.R. Kinder, T.M.Wong, and R. Meservey, "Large Magnetoresistance at Room Temperature in Ferromagnetic Thin Film Tunnel Junctions" Phys. Rev. Lett. 74, 3273 (1995).

[21] P. LeClair, J. T. Kohlhepp, C. H. van de Vin, H. Wieldraaijer, H. J. M. Swagten, W.J.M. de Jonge, A. H. Davis, J. M. MacLaren, J. S. Moodera, and R. Jansen, "Band Structure and Density of States Effects in Co-Based Magnetic Tunnel Junctions" Phys. Rev. Lett. 88, 107201 (2002).

[22] J. S. Moodera, J. Nowak, and R. J. M. van de Veerdonk, "Interface Magnetism and Spin Wave Scattering in Ferromagnet-Insulator-Ferromagnet Tunnel Junctions" Phys. Rev. Lett. 80, 2941 (1998).

[23] S. Yuasa, T. Nagahama, and Y. Suzuki, "Spin-Polarized Resonant Tunneling in Magnetic Tunnel Junctions" Science 297, 234 (2002).

[24] D. Wang, C. Nordman, J. M. Daughton, Z. Qian, and J. Fink, "70\% TMR at Room Temperature for SDT Sandwich Junctions With CoFeB as Free and Reference Layers" IEEE Trans. Magn. 40, 2269 (2004).

[25] J. J. Yang, C. Ji, Y. A. Chang, X. Ke, and M. S. Rzchowski, "Over 70\% tunneling magnetoresistance at room temperature for a CoFe and AlOx based magnetic tunnel junction" Appl. Phys. Lett. 89, 202502 (2006).

[26] A. M. Bratkovsky, "Tunneling of electrons in conventional and half-metallic systems: Towards very large magnetoresistance" Phys. Rev. B 56, 2344 (1997).

[27] A. M. Bratkovsky, "Assisted tunneling in ferromagnetic junctions and half- metallic oxides" Appl. Phys. Lett. 72, 2334 (1998).

[28] J. M. De Teresa, Barth, eacute, A. my, egrave, A. Fert, J. P. Contour, F. Montaigne, ccedil, ois, and P. Seneor, "Role of Metal-Oxide Interface in Determining the Spin Polarization of Magnetic Tunnel Junctions" Science 286, 507 (1999).

[29] C. T. Tanaka, J. Nowak, and J. S. Moodera, "Spin-polarized tunneling in a half- metallic ferromagnet" J. Appl. Phys. 86, 6239 (1999).

[30] W. H. Butler, X.-G. Zhang, T. C. Schulthess, and J. M. MacLaren, "Spin- dependent tunneling conductance of Fe $|\mathrm{MgO}| \mathrm{Fe}$ sandwiches" Phys. Rev. B 63, 054416 (2001).

[31] J. Mathon and A. Umerski, "Theory of tunneling magnetoresistance of an epitaxial $\mathrm{Fe} / \mathrm{MgO} / \mathrm{Fe}$ (001) junction" Phys. Rev. B 63, 220403 (2001).

[32] S. S. P. Parkin, C. Kaiser, A. F. Panchula, P. Rice, M. G. Samant, S.-H. Yang, and B. Hughes, "Giant tunneling magnetoresistance at room temperature with MgO (100) tunnel barriers" Nature Materials 3, 862 (2004). 
[34] S. Yuasa, T. Nagahama, A. Fukushima, Y. Suzuki, and K. Ando, "Giant roomtemperature magnetoresistance in single-crystal $\mathrm{Fe} / \mathrm{MgO} / \mathrm{Fe}$ magnetic tunnel junctions" Nature Materials 3, 868 (2004).

[35] S. Ikeda, J. Hayakawa, Y. Ashizawa, Y. M. Lee, K. Miura, H. Hasegawa, M. Tsunoda, F. Matsukura, and H. Ohno, "Tunnel magnetoresistance of $604 \%$ at $300 \mathrm{~K}$ by suppression of Ta diffusion in $\mathrm{CoFeB} / \mathrm{MgO} / \mathrm{CoFeB}$ pseudo-spin-valves annealed at high temperature" Appl. Phys. Lett. 93, 082508 (2008).

[36] R. Meservey and P. M. Tedrow, "Spin-polarized electron tunneling" Phys. Rep. 238, 173 (1994).

[37] C. Kaiser, S.van Dijken, S.H.Yang, H.Yang, and S.S.P. Parkin, "Role of Tunneling Matrix Elements in Determining the Magnitude of the Tunneling Spin Polarization of $3 d$ Transition Metal Ferromagnetic Alloys" Phys. Rev. Lett. 94, 247203 (2005).

[38] E.Y. Tsymbal, K. D. Belashchenko, J.P. Velev, S. S. Jaswal, M.v. Schilfgaarde, I. I. Oleynik, and C.A. Stewart, "Interface effects in spin-dependent tunneling" Prog. Mater. Sci. 52, 401 (2007).

[39] L. Berger, "Emission of spin waves by a magnetic multilayer traversed by a current" Phys. Rev. B 54 (13), 9353 (1996).

[40] J. C. Slonczewski, "Current-driven excitation of magnetic multilayers" J. Magn. Magn. Mater. 159, L1-L7 (1996).

[41] M.D. Stiles and J. Miltat, "Spin-transfer torque and Dynamics" Spin Dynamics in Confined Magnetic Structures III, Springer, 2006.

[42] D. Ralph and M. Stiles, “Spin transfer torques” J. Magn. Magn. Mater. 30 ( 008).

[43] J. A. Katine, F. J. Albert, R. A. Buhrman, E. B. Myers, and D. C. Ralph, "Current- Driven Magnetization Reversal and Spin-Wave Excitations in $\mathrm{Co} / \mathrm{Cu} / \mathrm{Co}$ Pillars" Phys. Rev. Lett. 84 (14), 3149-3152 (2000).

[44] E. B. Myers, D. C. Ralph, J. A. Katine, R. N. Louie, and R. A. Buhrman, "Current- Induced Switching of Domains in Magnetic Multilayer Devices" Science 285, 867-870 (1999).

[45] M. Tsoi, A. G. M. Jansen, J. Bass, W.-C. Chiang, V. Tsoi, and P. Wyder, "Generation and detection of phase-coherent current-driven magnons in magnetic multilayers" Nature 406, 46 (2000).

[46] J. Z. Sun, "Spin-current interaction with a monodomain magnetic body: A model study" Phys. Rev. B 62 (1), 570 (2000).

[47] J. Xiao, A. Zangwill, and M. D. Stiles, "Macrospin models of spin transfer dynamics" Phys. Rev. B 72 (1), 014446 (2005).

[48] J. Z. Sun, "Spin-current interaction with a monodomain magnetic body: A model study"

Phys. Rev. B 62, 570 (2000).

[49] R. H. Koch, J. A. Katine, and J. Z. Sun, "Time-resolved Reversal of Spin-Transfer Switching in a Nanomagnet."Phys. Rev. Lett. 92, 088302 (2004); 
[50] J. Z. Sun, “Spin angular momentum transfer in current-perpendicular nanomagnetic junctions.” IBM J. Res. \& Dev. 50, 81 (2006).

[51] Koji Tsunekawa, David D. Djayaprawira, Motonobu Nagai, Hiroki Maehara, Shinji Yamagata, and Naoki Watanabe, "Giant tunneling magnetoresistance effect in lowresistance $\mathrm{CoFeB} / \mathrm{MgO}(001) / \mathrm{CoFeB}$ magnetic tunnel junctions for read-head applications.”Appl. Phys. Lett. 87, 072503 (2005).

[52] Shinji Yuasa, Akio Fukushima, Hitoshi Kubota, Yoshishige Suzuki, Koji Ando,” Giant tunneling magnetoresistance up to $410 \%$ at room temperature in fully epitaxial Co/MgO/Co magnetic tunneljunctions with bcc Co(001) electrodes.” Appl. Phys. Lett. 89, 042505 (2006). [53] Jianguo Wang, P. P. Freitas, "low-current blocking temperature writing of double barrier magnetic random access memory cells.” Appl. Phys. Lett. 84, 945 (2004).

[54] S.Keshavarz, Y.Xu, S. Hrdy, C. Lemley, T. Mewes, Y. Bao, “Relaxation of Polymer Coated $\mathrm{Fe}_{3} \mathrm{O}_{4}$ Magnetic Nanoparticles in Aqueous Solution.” IEEE Transactions on magnetics, 2010.46:p.1541.

[56] Y. M. Lee, J. Hayakawa, S. Ikeda, F. Matsukura, and H. Ohno, “Effect of electrode composition on the tunnel magnetoresistance of pseudo-spin-valve magnetic tunnel junction with a MgO tunnel barrier.” Appl. Phys. Lett. 90, 212507 (2007).

[57] Z. Diao, D. Apalkov, M. Pakala, Y. Ding, A. Panchula, and Y. Huai,” Low magnetisation alloys for in-plane spin transfer torque devices.” Appl. Phys. Lett. 87(23), 232502-232503 (2005). [58] S. Ikeda, J. Hayakawa, Y. Ashizawa, Y. M. Lee, K. Miura, H. Hasegawa, M. Tsunoda, F. Matsukura, and H. Ohno, "Damping of Magnetization Precession in Perpendicularly Magnetized CoFeB alloy thin films.” Appl. Phys. Lett. 93(8), 082508 (2008).

[59] C. L. Wang, S. H. Huang, C. H. Lai, W. C. Chen, S. Y. Yang, K. H. Shen, and H. Y. Bor, "Perpendicular magnetization of Co2FeAl full-Heusler alloy films induced by MgO interface." J. Phys. D: Appl. Phys. 42, 115006 (2009).

[60] M. Oogane, T. Kubota, Y. Kota, S. Mizukami, H. Naganuma, A. Sakuma, and Y. Ando, "Gilbert magnetic damping constant of epitaxially grown Co-based Heusler alloy thin films." Appl. Phys. Lett. 96, 252501 (2010).

[61] K. Oguz, M. Ozdemir, O. Dur, and J. M. D. Coey, “Low magnetization alloys for in-plane spin transfer torque devices.” J. Appl. Phys. 111, 113904 (2012).

[62] Hitoshi Kubota, Akio Fukushima, Kay Yakushiji, Satoshi Yakata, Shinji Yuasa, Koji Ando, Mikihiko Ogane, Yasuo Ando, and Terunobu Miyazaki, “ Reduction in switching current using a low-saturation magnetization Co-Fe-(Cr,V)-B free layer in MgO-based magnetic tunnel junctions.” J. Appl. Phys. 105, 07D117 (2009).

[63] P. Gupta, Tapas Ganguli, A. Gupta, A. K. Sinha, S. K. Deb,” Effect of Co addition on the atomic ordering of FeCo-phase in nanocrystalline FeCoNbB alloys.” J. Appl. Phys. 111, 113518 (2012).

[64] Vsevolod I. Razumovskiy, Andrei V. Ruban, and Pavel A. Korzhavyi, “First-principles study of elastic properties of Cr-and Fe-rich Fe-Cr alloys.” Phys. Rev. B 84, 024106 (2011). [65] Yung-Hung Wang, Wei-Chuan Chen, Shan-Yi Yang, and Kuei-Hung Shen, "Interfacial and annealing effects on magnetic properties of CoFeB thin films.” J. Appl. Phys. 99, 08M307 (2006). 
[66]J. D. Livingston, “A review of coercivity mechanisms.” J. Appl. Phys., 52, 2544(1981). [67] S. Jin, D. Brasen, and S. Mahajan, “Coercivity mechanisms in Fe-Cr-Co magnet alloys.” J. Appl. Phys., 53(6), 4300(1982).

[68] Tsung-Shune Chin, Kou-Her Wang and Cheng-Hsiung Lin, "High Coercivity Fe-Cr-Co thin films by Vacuum evaporation.” Japanese Journal of Applied Physics, 30, 1692(1991).

[69] H. Meng, and J. P. Wang, "Spin transfer in nanomagnetic devices with perpendicular anisotropy.” Appl. Phys. Lett. 88, 172506 (2006).

[70] D. Houssameddine, U. Ebels, B. Delaët, B. Rodmacq, I. Firastrau, F. Ponthenier, M. Brunet, C. Thirion, J.P. Michel, L. Prejbeanu-Buda, M.C. Cyrille, O. Redon, and B. Dieny, "Spin-torque oscillator using a perpendicular polarizer and a planar free layer." Nature Matter. 6, 447 (2007).

[71] S. Mangin, D. Ravelosona, J. A. Katine, M. J. Carey, B. D. Terris, and E. E. Fullerton, "Current-induced magnetization reversal in nanopillars with perpendicular anisotropy." Nature Mater. 5, 210 (2006).

[72] K. Mizunuma, S. Ikeda, J. H. Park, H. Yamamoto, H. Gan, K. Miura, H.Hasegawa, J. Hayakawa, F. Matsukura, and H. Ohno, “MgO barrier-perpendicular magnetic tunnel junctions with CoFe/Pd multilayers and ferromagnetic insertion layers.” Appl. Phys. Lett.95, 232516 (2009).

[73] G. Kim, Y. Sakuraba, M. Oogane, Y. Ando, and T. Miyazaki, “Tunneling magnetoresistance of magnetic tunnel junctions using perpendicular magnetization L10-CoPt electrodes.” Appl. Phys. Lett. 92, 172502 (2008).

[74] Hideto Ohmori, Tomoya Hatori, and Shigeki Nakagawa, "Perpendicular magnetic tunnel junction with tunneling magneteoresistance ratio of 64\% using MgO (100) barrier layer prepared at room temperature.” J. Appl. Phys. 103, 07A911 (2008).

[75] S. Yakata, H. Kubota, Y. Suzuki, K. Yakushiji, A. Fukushima, S. Yuasa, K. Ando," Influence of perpendicular magnetic anisotropy on spin-transfer switching current in CoFeB./MgO/CoFeB magnetic tunnel junctions.” J. Appl. Phys. 105, 07D131 (2009).

[76] S. Ikeda, J. Hayakawa, Y. Ashizawa, Y. M. Lee, K. Miura, H. Hasegawa, M. Tsunoda, F. Matsukura, and H. Ohno, "Tunnel magnetoresistance of $604 \%$ at $300 \mathrm{~K}$ at suppression of Ta diffusion in $\mathrm{CoFeB} / \mathrm{MgO} / \mathrm{CoFeB}$ pseudo-spin-valves annealed at high temperature.” Appl. Phys. Lett. 93, 082508 (2008).

[77] M. Oogane, T. Kubota, Y. Kota, S. Mizukami, H. Naganuma, A. Sakuma, and Y. Ando, "Gilbert magnetic damping constant of epitaxially grown Co-based heusler alloy thin films." Appl. Phys. Lett. 96, 252501 (2010).

[78] H. Lee, Y.-H. A. Wang, C. K. A. Mewes, W. H. Butler, T. Mewes, S. Maat, B. York, M. J. Carey, and J. R. Childress, “Magnetization relaxation and structure of CoFeGe alloys.” Appl. Phys. Lett. 95, 082502 (2009).

[79] C. L. Wang, S. H. Huang, C. H. Lai, W. C. Chen, S. Y. Yang, K. H. Shen, and H. Y. Bor, J. Phys. D: Appl. Phys. 42, 115006 (2009).

[80] S. Maat, M. J. Carey, and J. R. Childress, "Current perpendicular to the plane spin-valves with CoFeGe magnetic layers.” Appl. Phys. Lett. 93, 143505 (2008). 
[81] M. T. Johnson, P. J. H. Bloemen, F. J. A. den Broeder, and J. J. de Vries, “Magnetic anisotropy in metallic multilayers.” Rep. Prog. Phys. 59, 1409 (1996).

[82] S. Ikeda, K. Miura, H. Yamamoto, K. Mizunuma, H. D. Gan, M. Endo, S.Kanai, J. Hayakawa, F. Matsukura, and H. Ohno, “A perpendicular-anisotropy CoFeB-MgO magnetic tunnel junction.” Nature Mater.9, 721 (2010).

[83] H. J. G. Draaisma, W. J. M. deJonge, and F. J. A. denBroeder, “Magnetic interface anisotropy in Pd/Co and Pd/Fe multilayers.” J. Magn. Magn. Mater. 66, 351 (1987).

[84] Riki Shimabukuro, KohjiNakamura, ToruAkiyama, Tomonorilto, "Electric field effects on magnetocryustalline anisotropy in ferromagnetic Fe monolayers.” Physica E 42,1014 1017 (2010).

[85] A. Manchon, C. Ducruet, L. Lombard, S. Auffret, B. Rodmacq, B. Dieny, S. Pizzini, J. Vogel, V. Uhlír, M. Hochstrasser, and G. Panaccione, "Analysis of oxygen induced anisotropy crossover in Pt/Co/MOx trilayers.” J. Appl. Phys. 104, 043914 (2008).

[86] R. D. McMichael, M. D. Stiles, P. J. Chen, and W. F. Egelhoff, “Ferromagnetic Resonance line width in thin films.” J. Appl. Phys. 83, 7037 (1998).

[87] E. Krén and G. Kádár, Solid State Commun. 8, 1653 (1970).

[88] B. Balke, G. H. Fecher, J. Winterlik, and C. Felser, “ $M n_{3} G a$, a compensated ferromagnetic with high Curie temperature and low mangetic moment for spin torque transfer applications.” Appl. Phys. Lett. 90, 152504 (2007).

[89] H. Masumoto, K. Watanabe, and M. Mitera, J. Jpn. Inst. Met. 42, 474 (1978).

[90] H. Niida, T. Hori, H. Onodera, Y. Yamaguchi, and Y. Nakagawa, "Magnetization and coercivity of Mn3- $\delta G$ a alloys with a D022-type structure.” J. Appl. Phys. 79, 5946 (1996). [91] G. Kim, Y. Sakuraba, M. Oogane, Y. Ando, and T. Miyazaki, “Tunneling Magnetoresistance of magnetic tunnel junctions using perpendicular magnetization L1CoPt electrodes.” Appl. Phys. Lett. 92, 172502 (2008).

[92] N. Nishimura, T. Hirai, A. Koganei, T. Ikeda, K. Okano, Y. Sekiguchi, and Y. Osada, “ Magnetic tunnel junction device with perpendicular magnetization films for high-density magnetic random access memory.” J. Appl. Phys. 91, 5246 (2002).

[93] T. Seki, T. Shima, K. Takanashi, Y. Takanashi, E. Matsubara, and K. Hono, “L1。 ordering of off-stoichiometric FePt (001) thin films at reduced temperature.” Appl. Phys. Lett. 82, 2461 (2003).

[94] A. D. Kent, B. Ozyilmaz, and E. del Barco, “Spin-transfer Induced precessional magnetization reversal.” Appl. Phys. Lett. 84, 3897 (2004).

[95] M. Nakayama, T. Kai, N. Shimomura, M. Amano, E. Kitagawa, T. Nagase, M. Yoshikawa, T. Kishi, S. Ikegawa, and H. Yoda,"Spin transfer switching in $\mathrm{TbCoFe} / \mathrm{CoFeB} / \mathrm{MgO} / \mathrm{CoFeB} / \mathrm{TbFeCo}$ magnetic tunnel junctions with perpendicular magnetic anisotropy.” J. Appl. Phys.103, 07A710 (2008).

[96] Ching-Ming Lee, Lin-Xiu Ye, Jia-Mou Lee, Wan-Ling Chen, Chao-Yuan Huang, Gung Chern, and Te-Ho Wu, “Ultrathin (Gd, Tb)-FeCo films with perpendicular Magnetic Anisotropy.” IEEE Trans. Magn. 45, 3808 (2009). 
[97] T. Hauet, F. Montaigne, M. Hehn, Y. Henry, and S. Mangin, “Influence of an interface domain wall on spin-valve giant magnetoresistance.” Appl. Phys. Lett. 93, 222503 (2008). [98] P. Hansen, J. Magn. Magn. Mater. 83, 6 (1990).

[99] O. S. Anilturk and A. R. Koymen, “Element-specific magnetization of a Gd-Co composite system using spin-polarized Auger electron spectroscopy.” Phys. Rev. B 68, 024430 (2003). [100] Y. Mimura, N. Imamura, T. Kobayashi, A. Okada, and Y. Kushiro, J. Appl. Phys. 49, 1208 (1978).

[101] X. J. Bai, J. Du, J. Zhang, B. You, L. Sun, W. Zhang, X. S. Wu, S. L. Tang, and A. Hu, "Giant magnetoresistance and tunnel magnetoresistance effects in FeCoGd-based spin valves and magnetic tunnel junctions.” J. Appl. Phys. 103, 07F305 (2008).

[102] M. T. Johnson, P. J. H. Bloemen, F. J. A. den Broeder, and J. J. de Vries, "Magnetic anisotropy in metallic multilayers.” Rep. Prog. Phys. 59, 1409 (1996).

[103] Thomas A. Ostler, Richard F. L. Evans, and Roy W. Chantrell, Unai Atxitia, Oksana Chubykalo- Fesenko, Ilie Radu, Radu Abrudan, Florin Radu, Arata Tsukamoto, A. Itoh, Andrei Kirilyuk, Theo Rasing, and Alexey Kimel, "Crystallographically amorphous ferromagnetic alloys: Comparing a localized atomistic spin model with experiments.” Phys. Rev. B 84, 024407 (2011).

[104] C. D. Stanciu, A. V. Kimel, F. Hansteen, A. Tsukamoto, A. Itoh, A. Kirilyuk and Th. Rasing, "Ultrafast spin dynamics across compensation points in ferrimagetic GdFeCo: The role of angular momentum compensation.” Phys. Rev. B 73, 220402(R) (2006).

[105] H. J. Leamy and A. G. Dirks, "Microstructure and Magnetism in amorphous rare-earthtransition-metal thin films." J. Appl. Phys. 50 (4), 2871 (1979).

[106] V. G. Harris, K. D. Aylesworth, B. N. Das, W. T. Elam, and N. C. Koon, "Structural origins of magnetic anisotropy in sputtered amorphous Tb-Fe films." Phys. Rev. Lett. 69, 1939 (1992).

[107] X. Yan, M. Hirscher, T. Egami, and E. E. Marinero, “Direct observation of anelastic bond-orientational anisotropy in amorphous TbFeCo thin films by $X$-ray diffraction.” Phys. Rev. B 43, 9300 (1991).

[108] R. Shan, J. Du, X. X. Zhang, L. Sun, W. W. Lin, H. Sang, T. R. Gao, and S. M. Zhou, “Antiferromagnetic coupling and perpendicular anisotropy in TbFeCo/NiO multilayers." Appl. Phys. Lett. 87, 102508 (2005). 